\title{
terestirycis
}

Universidad Autónoma

de Madrid

This paper must be cited as:

Marin, R.; Jaque, D.; Chem. Rev. 2021, 121, 3, 1425-1462, DOI:

10.1021/acs.chemrev.0c00692

Doping Lanthanide lons in Colloidal Semiconductor Nanocrystals for Brighter Photoluminescence

Riccardo Marin, ${ }^{1}$ Daniel Jaque ${ }^{1}$

${ }^{1}$ Fluorescence Imaging Group, Departamento de Física de Materiales - Facultad de Ciencias, Universidad Autónoma de Madrid, C/Francisco Tomás y Valiente 7, Madrid 28049, Spain

E-mail: daniel.jaque@uam.es

This document is the unedited Author's version of a Submitted Work that was subsequently accepted for publication in Chemical Reviews, copyright (C) American Chemical Society after peer review. To access the final edited and published work see:

https://pubs.acs.org/doi/10.1021/acs.chemrev.0c00692 


\section{Doping Lanthanide lons in Colloidal Semiconductor Nanocrystals for Brighter Photoluminescence}

Riccardo Marin ${ }^{a *}$, Daniel Jaque ${ }^{a, b *}$

a Fluorescence Imaging Group (FIG), Departamento de Física de Materiales, Facultad de Ciencias, Universidad Autónoma de Madrid, C/ Francisco Tomás y Valiente 7, Madrid 28049, Spain. *riccardo.marin@hotmailit, daniel.jaque@uam.es.

b Nanobiology Group, Instituto Ramón y Cajal de Investigación, Sanitaria Hospital Ramón y Cajal, Ctra. De Colmenar Viejo, Km. 9100, 28034 Madrid, Spain

\section{Table of Contents:}

Abstract. 3

1. Introduction. 3

2. Why doping lanthanide ions in semiconductors? $\quad 7$

2.1. Lanthanide ions.

2.2. Semiconductor nanocrystals and quantum dots. 8

2.3. Doping lanthanide ions in semiconductor nanocrystals. 9

3. Designing a lanthanide-doped semiconductor nanocrystal. 10

$\begin{array}{ll}\text { 3.1. Chemical considerations. } & 10\end{array}$

3.1.1. Cation site and coordination environment. 10

3.1.2. Ionic radius and oxidation state. 11

3.1.3. Divalent europium. 12

3.1.4. Summary. 13

3.2 Physical considerations. 13

$\begin{array}{ll}\text { 3.2.1. Bandgap and interbandgap states. } & 13\end{array}$

$\begin{array}{ll}\text { 3.2.2. Charge transfer. } & 15\end{array}$

$\begin{array}{ll}\text { 3.2.3. Cation site symmetry. } & 17\end{array}$

3.2.4. Use of lanthanides with $4 \mathrm{f}-5 \mathrm{~d}$ transitions $\left(\mathrm{Ce}^{3+}\right.$ and $\left.\mathrm{Eu}^{2+}\right)$. 17

$\begin{array}{ll}\text { 3.2.5. Summary. } & 18\end{array}$

3.3. Additional observations on the design. 19

4. Synthesis strategies $\quad 21$

4.1. General considerations $\quad 21$

4.2. A game of precursor reactivity control. 22

4.3. Surface engineering. 25

5. Characterization techniques.

5.1. Structural and compositional characterization. 27

5.2. Light interaction and generation. 29

6. Case studies.

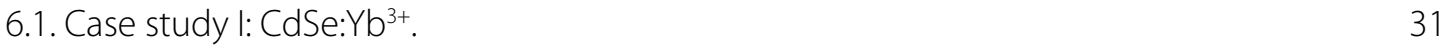

6.2. Case study II: $\mathrm{Pb}_{2} \mathrm{n}_{2} \mathrm{~S}_{4}: \mathrm{Yb}^{3+}$.

6.3. Case study III: $\mathrm{CsPbX}_{3}: \mathrm{Yb}^{3+}$ (and other halide perovskites). 33

6.3.1. Lead-based perovskites. 33

6.3.2. Lead-free perovskites. 36

6.3.3. Lanthanide-based perovskites $\quad 36$

6.4. Case study IV: Alkaline earth sulfides. 
7. Applications.

7.1. Photovoltaics.

7.2. Photodetectors. 41

7.3. Light-emitting diodes. 41

7.4. Sensing. $\quad 42$

7.5. Biomedical 42

8. Perspectives and outlook.

Acknowledgments.

References. $\quad 45$ 


\begin{abstract}
The spectrally narrow, long-lived luminescence of lanthanide ions makes optical nanomaterials based on these elements uniquely attractive from both a fundamental and applicative standpoint. A highly coveted class of such nanomaterials is represented by colloidal lanthanide-doped semiconductor nanocrystals (LnSNCs). Therein, upon proper design, the poor light absorption intrinsically featured by lanthanides is compensated by the semiconductor moiety, which harvests the optical energy and funnel it to the luminescent metal center. Although a great deal of experimental effort has been invested to produce efficient nanomaterials of that sort, relatively modest results have been obtained thus far. As of late, halide perovskite nanocrystals have surged as materials of choice for doping lanthanides, but they have non-negligible shortcomings in terms of chemical stability, toxicity, and light absorption range. The limited gamut of currently available colloidal LnSNCs is unfortunate, given the tremendous technological impact that these nanomaterials could have in fields like biomedicine and optoelectronics. In this Review, we provide an overview of the field of colloidal LnSNCs, while distilling the lessons learnt in terms of material design. The result is a compendium of key aspects to consider when devising and synthesizing this class of nanomaterials, with a keen eye on the foreseeable technological scenarios where they are poised to become front runners.
\end{abstract}

\title{
1. Introduction
}

A recent essay by one of the most esteemed scientists in the field of lanthanides, Prof. Bünzli, labelled materials based on these elements "Rising Stars in Science and Technology". ' We are far from the times when this series of elements was - we dare to say - belittled: $:^{*}$ nowadays, technologies like anti-counterfeiting, lighting, and permanent magnets hinge on the optical and magnetic properties of lanthanide ions. ${ }^{1,3,4}$ Hence, although the charming properties of these ions had remained elusive for a long time, lanthanide-based materials are now the focus of the investigation of numerous research groups around the world.

The properties of lanthanide ions stem from their electronic structure, which allows for unique behaviors to arise, such as upconversion luminescence ${ }^{5,6}$ and single-ion magnetism., ${ }^{4,7}$ The knowledge around the physical mechanisms underpinning lanthanide ions' optical and magnetic features has dramatically increased over the past half century, ensuring an exquisite degree of control over them. Coincidentally, although the seed for the research field of the ultra-small was planted already in 1959 by Richard Feynman, it is in the past three decades that nanotechnology has experienced an incredible bloom. The natural consequence of the coexistence of these two effervescent fields, namely lanthanidebased materials and nanotechnology, was thus their marriage. And a very happy one.

To that end, a good example is offered by lanthanide-based luminescent colloidal nanomaterials: with their distinctive emission fingerprint, they are invaluable tools for the investigation of phenomena in the realm of the extremely small, 10 and extremely fast. ${ }^{11}$ They are currently investigated as probes in nanomedicine, for the development of all-optical diagnostic techniques. ${ }^{12-14}$ In the field of photovoltaics they can provide the additional boost to overcome the Shockley-Queisser limit. ${ }^{15}$, 16 Aside from the technological relevance of nanomaterials based on these elements, as scientists we are also genuinely mesmerized by their luminescence: the complex physical processes governing it are source of continue wonder, pushing us to a pursue their deeper understanding.

However, colloidal lanthanide-based luminescent nanomaterials suffer from a major limitation: the direct light absorption by lanthanide ions is intrinsically poor. This results in an overall low

\footnotetext{
"In their book "Understanding Chemistry", Pimentel and Sprately commented how "Lanthanum has only one important oxidation state in aqueous solution, the +3 state. With few exceptions, this tells the whole boring story about the other 14 Lanthanides".
} 
photoluminescence brightness (roughly put, the product of absorption and emission efficiency), which is the real Achille's heel of these materials. Exceptions to this general behavior of lanthanide ions are represented by some transitions in divalent and trivalent species like $\mathrm{Eu}^{2+}, \mathrm{Ce}^{3+}, \mathrm{Sm}^{2+}$, and some dedicated considerations regarding these ions are made in this Review where relevant.

Different strategies have been developed to overcome the hurdle of poor emission brightness (Table 1), such as the engineering of complex core/multiple-shells architectures and coupling with highly-absorbing moieties including plasmonic nanoparticles, ${ }^{17,}{ }^{18}$ organic molecules, ${ }^{19,} 20$ or semiconductor nanocrystals (SNCs) ${ }^{21,22}$. The first strategy leverages the concept of energy transfer from a sensitizer (the ion absorbing the optical energy) to an activator (the ion to which the absorbed energy is transferred and that re-emits it in the form of photon). The use of core/shell architectures generally aims to increase the total number of sensitizers per particle, minimize the losses during the sensitizerto-activator energy transfer processes, and limits surface quenching phenomena. ${ }^{23,24}$ Unfortunately, the low absorption coefficient of lanthanide ions poses an intrinsic limit to this approach. The latter strategies make use of an additional species that is tethered to - or grown as a shell on - a pre-existing lanthanidebased nanoparticle. The use of plasmonic moieties is a powerful approach, which allows to increase both the absorption cross section of $\mathrm{Ln}^{3+}$ as well as the probability of radiative decay - depending on whether the plasmonic resonance overlaps with the absorption or emission lines. ${ }^{17,25}$ These two effects result respectively from the local enhancement of the electromagnetic field (excitation photons) and the socalled Purcell effect, which entails a local density of states increase, ultimately leading to an increased rate of emission. The limitation of this approach is that no broadband absorption is achievable, with the absorption wavelength being dictated by the $\mathrm{Ln}^{3+}$ employed. ${ }^{26}$ Organic dyes and SNCs offer instead broadband absorption capabilities and tunability of the absorption range. ${ }^{20-22}$ Strategies based on the use of these moieties leverage the same principle: absorption of the energy in the form of photons by the donor (dye/SNC) and its funneling to the $\mathrm{Ln}^{3+}$ ions (acceptors) within the lanthanide-based nanoparticle mainly via a Förster resonance energy transfer (FRET) transfer process. Importantly, for effective FRET to occur, the donor should be as close as possible to the acceptor: an aspect that limits the applicability of these approaches in tandem with the growth of a protective shell - which reduced surface quenching phenomena (see Section 4.3), but also increases the donor-acceptor distance. ${ }^{27,28}$ The enhancement of the absorption achievable with these approaches is sizeable, but it does not directly translate to a betterment of the brightness of $\mathrm{Ln}^{3+}$ emission, since the additional step of energy transfer heavily impacts the final efficiency of sensitization. A further concern related with the use of dyes is their limited (photo)chemical stability and their poor compatibility with aqueous environments, which hampers the use of this sensitization pathway for the preparation of water-dispersible nanosystems.

A different method, used more in general for the preparation of bright lanthanide-based phosphors, relies on co-doping $\mathrm{Ln}^{3+}$ and other ions such as $\mathrm{Bi}^{3+29-32}, \mathrm{Cr}^{3+33-35}, \mathrm{Mn}^{3 / 4+36}$ or $\mathrm{Ce}^{3+37-41}$. These ions possess allowed electronic transitions, so that they can absorb the optical energy in a broadband fashion and funnel it to the emitting $\mathrm{Ln}^{3+}$ centers. Although the energy of those allowed transitions is dependent on the crystal field experienced by the ion, those absorption bands generally fall at the shorter-wavelength end of the visible spectrum - hence limiting the applicability of this strategy when absorption approaching near-infrared (NIR) is sought after. Absorption (and emission) in this longer-wavelength range is pivotal, for instance, in biomedical applications to ensure maximal penetration within biological tissues (vide infra). There is yet another approach, which capitalizes on the strong absorption featured by SNCs without the need to prepare a composite nanostructure: the synthesis of lanthanide-doped SNCS (LnSNCS). 
Table 1. Summary of the strategies for brightness enhancement of $\mathrm{Ln}^{3+}$ photoluminescence in colloidal nanoparticles. References to specific works in the literature are reported in the main text.

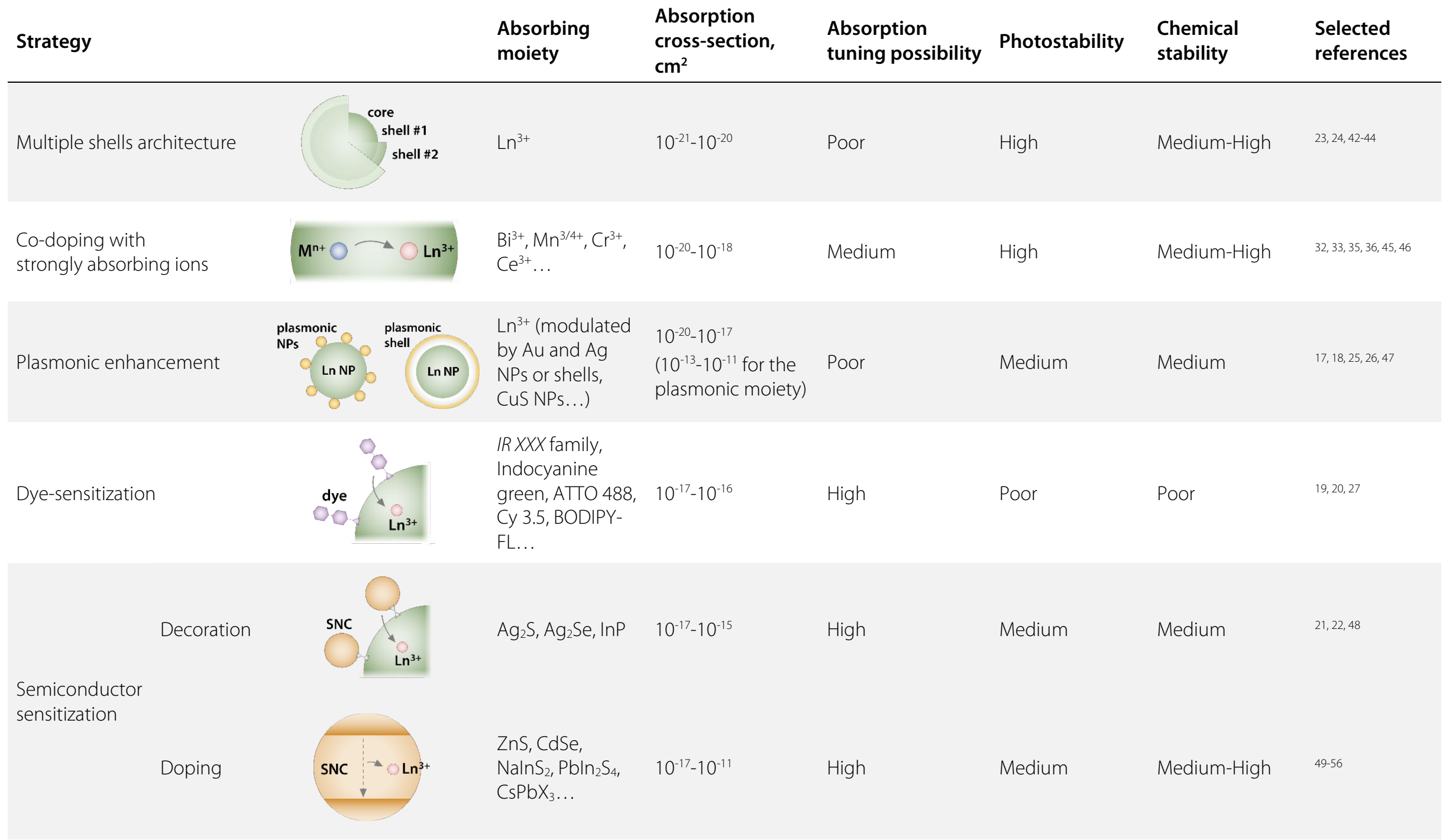


LnSNCs can be regarded as the all-inorganic counterpart of luminescent lanthanide complexes. In these latter species, the lanthanide ion is coordinated by organic molecules, which harvest the excitation light and transfer the energy to the lanthanide., ${ }^{3,57,58}$ In LnSNCs the role of light absorber is played by the SNC lattice in which the lanthanide ion is doped. The combined photophysical and chemical stability, along with bright lanthanide photoluminescence are the differentiators of LnSNCs, which have been sought after for many years now. Albeit some first encouraging results, we are not even close to the mastery achieved in the preparation and fine-tuning of the properties of lanthanide complexes. Admittedly, a steady honing of the tools at disposal of coordination chemists is being carried out for almost two centuries now. ${ }^{*}$ Nanotechnology is instead a much younger subfield of inorganic chemistry and, although great strides have been made, many dark corners are still present.

With this premise in mind, in this Review we take a close look to the recent advancements in the field of colloidal lanthanide-doped semiconductor nanocrystals (Scheme 1). In Section 2, we provide a justification as of why the preparation of LnSNC is an appealing and versatile strategy to enhance the brightness of lanthanide ions' photoluminescence. We then briefly introduce the properties of lanthanide ions and semiconductors, laying the foundations for the subsequent sections that deal with the three main aspects of LnSNC development: material design (Section 3), synthesis methods (Section 4), and characterization of the material (Section 5). We focus our attention particularly on the material design, identifying the key aspects to consider when selecting a lanthanide-semiconductor pair from the standpoint of chemistry and physics of both moieties. We proceed to review the approaches developed so far for the synthesis of colloidal LnSNCs, highlighting those of interest for the specific case of lanthanide doping. Characterization techniques apt to check the successful incorporation of lanthanide ions in the semiconductor lattice are laid out, along with indications about how to extract the most relevant information they carry about the material.

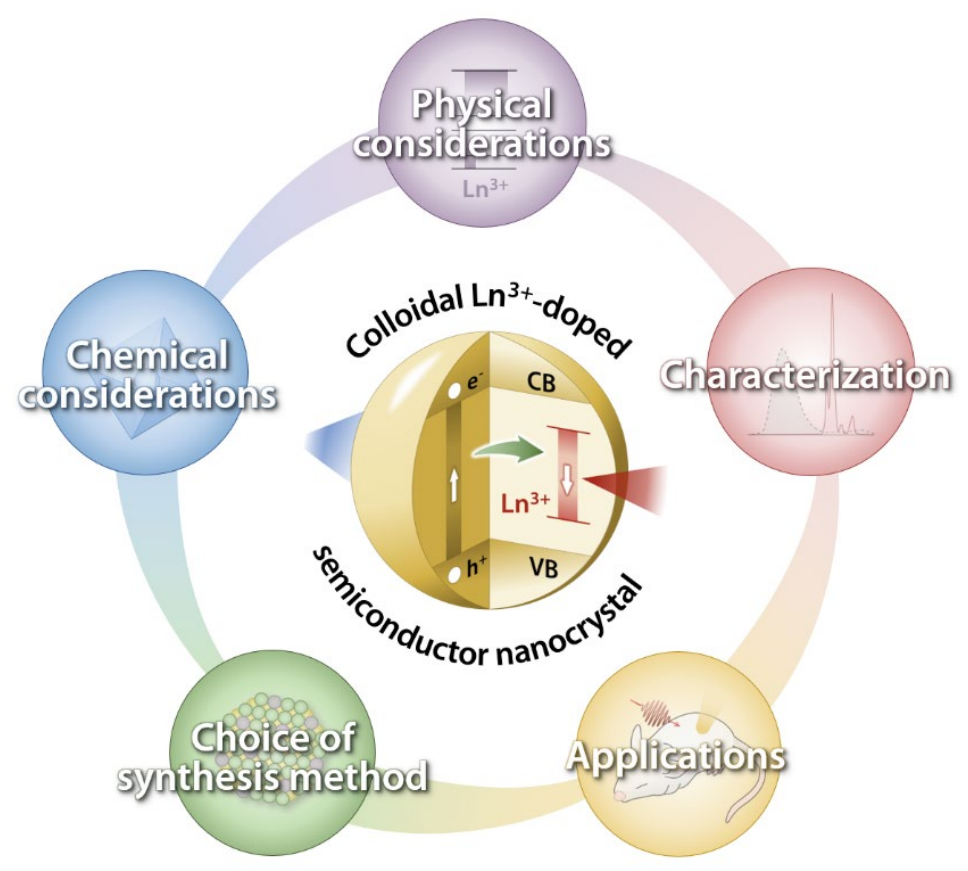

Scheme 1. Aspects discussed in this Review about colloidal Ln ${ }^{3+}$-doped semiconductor nanocrystals (LnSNCs).

\footnotetext{
*In 1798, B. M. Tassaert reported the first coordination compound of cobalt and ammonia without, however, fully understanding the material. Passing through S. M. Jörgensen's chain theory to explain metal complexes, it was only in 1893 that A. Werner finally realized the existence of a principal (oxidation state) and auxiliary (coordination number) for the metal in that "odd" class of complex compounds (as Tassaert first referred to them).
} 
In Section 6, we present illustrative case studies, which we analyze meticulously in light of the most relevant aspects discussed throughout the Review. Section 7 deals with the proposed applications of the most recently developed LnSNCs. An outlook on applications where these systems are poised to make a difference is provided in Section 8, along with our opinions regarding the most promising paths that the research on the subject could undertake in the pursue of new, multifunctional LnSNCs.

Before plunging into the various aspects of design and synthesis of LnSNCs, a caveat is due: there is no universal recipe for such materials! Here we deconstruct the subject, tackling systematically each of its facets with the help of available experimental evidence from the literature. We select those we consider the most relevant works in the field and extract from there the lessons learnt. Rather than a compilation of studies on the subject, this Review should be regarded by the reader as a vade mecum for the preparation of colloidal lanthanide-doped semiconductor nanocrystals, where the main guidelines to maximize the chances of success are outlined.

\section{Why doping lanthanide ions in semiconductors?}

\subsection{Lanthanide ions}

With some exceptions, lanthanide ions are commonly found in their triply oxidized state $\left(L n^{3+}\right)$, with general electronic configuration [Xe] $4 f^{n}$. These electrons located in the $4 f$ orbitals are the responsible for the unique physical properties of $\mathrm{Ln}^{3+}$. In this Review, we mainly focus our attention on trivalent lanthanide ions, with some excursions in the world of divalent lanthanides when relevant. From a spectroscopic standpoint, the origin of $\mathrm{Ln}^{3+}$ optical properties from intraconfigurational $4 \mathrm{f}-4 \mathrm{f}$ electronic transitions is both the blessing and the curse of these elements. In fact, these transitions are forbidden by the Laporte rule (parity forbidden) and, as such, have low probability of occurring., 3,7 On top of that, $4 f$ orbitals are shielded by $5 s$ and $5 p$ sub-shells and do not participate in the formation of bonds with other elements. From a spectroscopic standpoint, the result is a set of radiative electronic transitions with narrow bandwidth (atom-like), extended lifetime (micro- to milliseconds), and whose energy is poorly influenced by the chemical environment. ${ }^{*}$ However, direct excitation of $\mathrm{Ln}^{3+}$ ions is an inefficient process, owing to the mentioned forbidden nature of the electronic transitions involved. A partial relaxation of the Laporte rule is achieved upon introduction the $\mathrm{Ln}^{3+}$ ion in a low-symmetry environment (i.e., non-centrosymmetric), which favors partial admixing of the $4 f$ orbitals with opposite-parity wavefunctions, such as those of $\mathrm{Ln}^{3+} 5 \mathrm{~d}$ orbitals or other anionic species to which the metal ion is bound. Yet, as we will see, there are more effective ways to increase the emission brightness of lanthanide ions than simply relying on low-symmetry coordination geometries.

Although most of the electronic transitions of lanthanides are characterized by the abovementioned traits, in some cases parity allowed transitions are observed. $\mathrm{Ce}^{3+}, \mathrm{Tb}^{3+}, \mathrm{Eu}^{2+}, \mathrm{Sm}^{2+}, \mathrm{Tm}^{2+}$ are some of the lanthanides that feature these electrical-dipole allowed transitions between $4 \mathrm{f}$ and $5 \mathrm{~d}$ orbitals. Given this, and the fact that $5 \mathrm{~d}$ orbitals are not shielded, $4 \mathrm{f}-5 \mathrm{~d}$ transitions feature broad bands, short lifetimes (up to tens of ns), and their energy is highly dependent upon the coordination environment. This latter feature makes the optical properties of those lanthanides tunable via host selection. The allowed nature of these electronic transitions translates to a stronger absorption capability as well as a more intense overall emission brightness.

Brightness is defined as the product between photoluminescence quantum yield (PLQY - number of emitted photons per absorbed excitation photons) and molar extinction coefficient. This parameter

\footnotetext{
"This last statement is only partially true. There are slight changes in the position of the $4 \mathrm{f}-4 \mathrm{f}$ emission lines of $\mathrm{Ln}^{3+}$ depending on the coordination environment of the ion. The extent of such shifts is of up to a few hundred wavenumbers at most and they occur because of the so-called nephelauxetic effect, where changes in the bond length between $\mathrm{Ln}^{3+}$ and the surrounding anions result in variation of $4 \mathrm{f}$ electronic distribution.
} 
thus quantifies the fraction of incident photons that are converted into emitted light. From the above definition, one infers that a viable strategy to enhance the brightness of $\mathrm{Ln}^{3+}$ ions is to exploit moieties with strong light absorption capability and that can funnel the absorbed energy to the metal ion. This approach is referred to as sensitization and the light-absorbing moiety is often called antenna. ${ }^{3,58}$ Here is where semiconductors come into play.

\subsection{Semiconductor nanocrystals and quantum dots}

Semiconductors are somewhat arbitrarily distinguished from insulators according to the magnitude of their bandgap, but the boundary between the two classes of materials is rather loose. In this Review, we treat as semiconductors all those materials with a bulk bandgap up to approximately $5 \mathrm{eV}$. This definition allows us to include compounds such as alkaline earth sulfides, which are borderline wide bandgap semiconductors (4.5-5.5 eV). ${ }^{60,61}$ Unlike $\mathrm{Ln}^{3+}$, semiconductors feature large extinction coefficients (up to $10^{6} \mathrm{~cm}^{-1}$ vs $0.1-10 \mathrm{~cm}^{-1}$ for $\mathrm{Ln}^{3+}$ ) over a broad wavelength range. The absorption onset depends on the material: different semiconductors start absorbing at different wavelengths depending on the energy required for each specific material to promote an electron from the valence to the conduction band (VB to $C B$, Figure 1A). The excited electron leaves behind a positively charged hole in the valence band. The stabilizing coulombic interaction between this pair of charge carriers (exciton) makes it possible for the material to absorb light of energy smaller than its electronic bandgap. This effect leads to the appearance of one or multiple peaks in the absorption spectrum of the material. The energy at which the first of these excitonic transitions is observed is referred to as the optical bandgap of the material. ${ }^{62}$ The recombination of hole and electron in the form of an exciton leads to the emission of a photon. ${ }^{63}$ This is a rather simplified picture, as other processes can come into play. For instance, there are several types of excitons (free, bound, self-trapped) ${ }^{64}$ and the recombination might occur with the involvement of interbandgap states arising from native lattice defects, such as anion and metal vacancies. Some of those situations are discussed where relevant throughout the Review.

When a semiconductor is shrunk to the nanoscale, quantum effects start coming into play. The size threshold at which these phenomena start becoming appreciable depends on the exciton Bohr radius of the semiconductor - a parameter that depends on the composition of the material. The exciton Bohr radius defines the size of the quasiparticle exciton (i.e., an electron-hole pair interacting electrostatically) that can travel throughout the material. ${ }^{63}$ When the radius of a SNC approaches the size of its exciton Bohr radius, quantum confinement effects starts arising and the SNC is referred to as a quantum dot. ${ }^{64}$ This size regime is characterized by discretization of the energy levels prominently at the edges of the electronic bands (Figure 1B). The result is the appearance of several peaks in the absorption spectrum of a quantum dot (see Ref. ${ }^{65}$ for a thorough explanation of this effect). The magnitude of this discretization is a function of the SNC size and it can be interpreted considering the particle-in-a-box model. ${ }^{65}$ It is interesting to note that, at this small size, the confinement experienced by the charge carriers is as important as the Coulomb interaction, or even dominant (when the SNC radius is smaller than the exciton Bohr radius). Because of quantum confinement, the bandgap of the nanocrystal can be controlled by changing its size (Figure 1C). ${ }^{66} \mathrm{It}$ is therefore clear that, depending on the magnitude of the exciton Bohr radius, not all SNCs can be defined as quantum dots. For instance, $A_{2} S$ is a semiconductor with bulk bandgap of $0.9-1.0 \mathrm{eV}$ and Bohr radius of $2 \mathrm{~nm} .{ }^{67}$ Because of their exciton Bohr radius, $\mathrm{Ag}_{2} \mathrm{~S}$ nanocrystals larger than $4 \mathrm{~nm}$ already feature the same bandgap of the bulk material: they are not in quantum confinement regime and thus they cannot be referred to as quantum dots in spite of their small size. In the present Review, we adopt the broader term SNCs.

The intrinsic limitation of optical tuning via size variation is that, given a material (hence the bulk bandgap), one can only increase the distance between the electronic bands of the SNC. On top of that, the smaller the SNC the higher the surface-to-volume ratio. Since surface defects and interaction with 


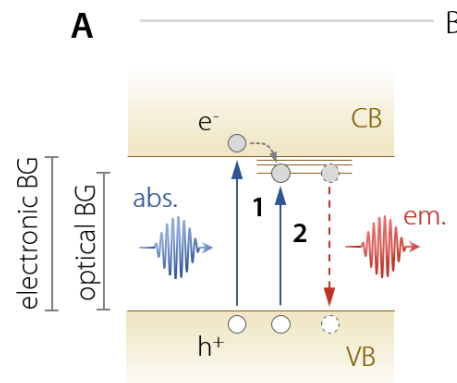

Bulk

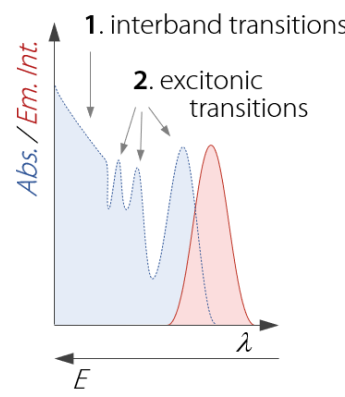

B Quantum dot
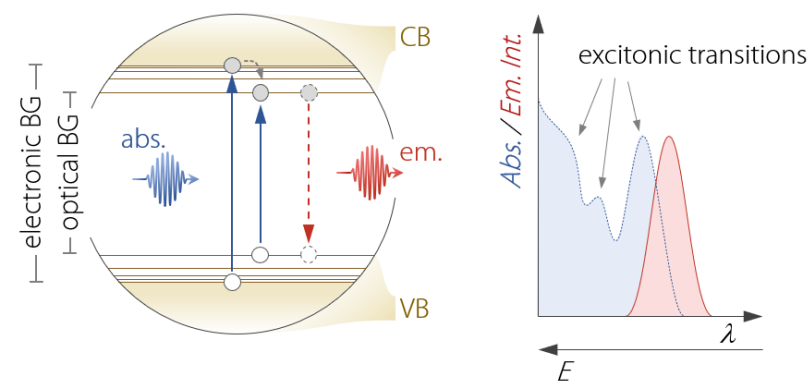

C

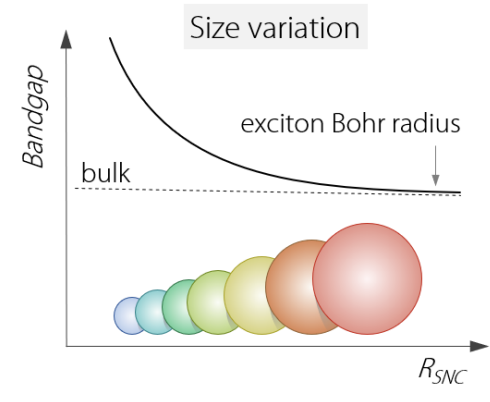

D

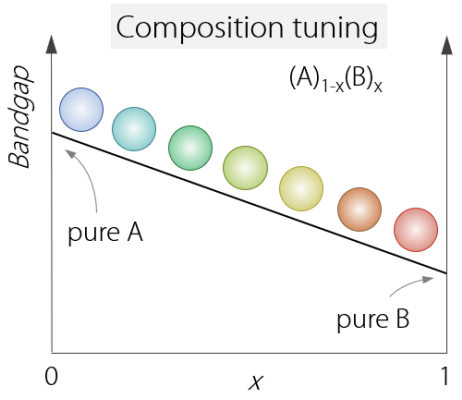

Figure 1. Mechanism of electron (e) photoexcitation and its recombination with a hole $\left(\mathrm{h}^{+}\right)$in bulk semiconductor (A) and in a SNC in quantum confinement regime (quantum dot - B), along with the respective characteristic absorption and emission spectra. Solid blue arrows - absorption phenomena; dashed red arrows - radiative recombination. VB - valence band; CB - conduction band. The bandgap of a SNC can be tailored exploiting strategies such as size control (C - when in quantum confinement regime) and composition tuning (D).

molecules adsorbed at the nanocrystal surface are amongst the main responsible for non-radiative recombination of charge carriers (see Section 4), ${ }_{1}^{68}$ preparing small nanocrystals is not always convenient. Another approach for engineering the bandgap of the material is to alloy the selected semiconductor (A) with another miscible semiconductor (B) that has a different bandgap (Figure 1D). ${ }^{69}$ This strategy is employed for several combinations of semiconductors, among which $\mathrm{CulnS}_{2}-\mathrm{ZnS}^{70,71}$ and $\mathrm{CsPb}\left(\mathrm{Cl}_{x} \mathrm{Br}_{1}\right.$ $x)_{3}{ }^{72}$. The lattice parameters of the mixed material generally follow the so-called Vegard's law, with an approximately linear dependence on the fraction of one of the alloyed materials. ${ }^{73,74}$ This trend is mirrored by the magnitude of the bandgap, ${ }^{75}$ which also scales linearly with the amount of alloyed material, although deviations from the linear trend (bowing) are often observed. ${ }^{76}$ This method can be combined with size tuning to achieve an even greater degree of control over the SNC bandgap.

The fact that alloying with a secondary material introduces variation in the bandgap of the system is particularly relevant in this context. When one introduces $\mathrm{Ln}^{3+}$ ions in a lattice, the metal's orbitals involved in the bonds contribute to the band structure of the host materials, also introducing interbandgap states. While it is reasonable to assume that the former effect is minimal or negligible for low doping percentages, localized defects (and associated trap states) are generally formed already when as little as one single dopant is introduced in the lattice. 77,78

\subsection{Doping lanthanide ions in semiconductor nanocrystals}

$\mathrm{Ln}^{3+}$-doped semiconductor nanocrystals ( $\mathrm{LnSNCS}$ ) capitalize on the capability of semiconductors to effectively absorb the excitation light and, upon proper tailoring of the system, sensitize the emission of $\mathrm{Ln}^{3+}$ ions embedded in the crystalline host (Figure 2). The result is a single entity in the form of a nanoparticle with unparalleled combination of bright, long-lived, and spectrally narrow $\mathrm{Ln}^{3+}$ emission, 

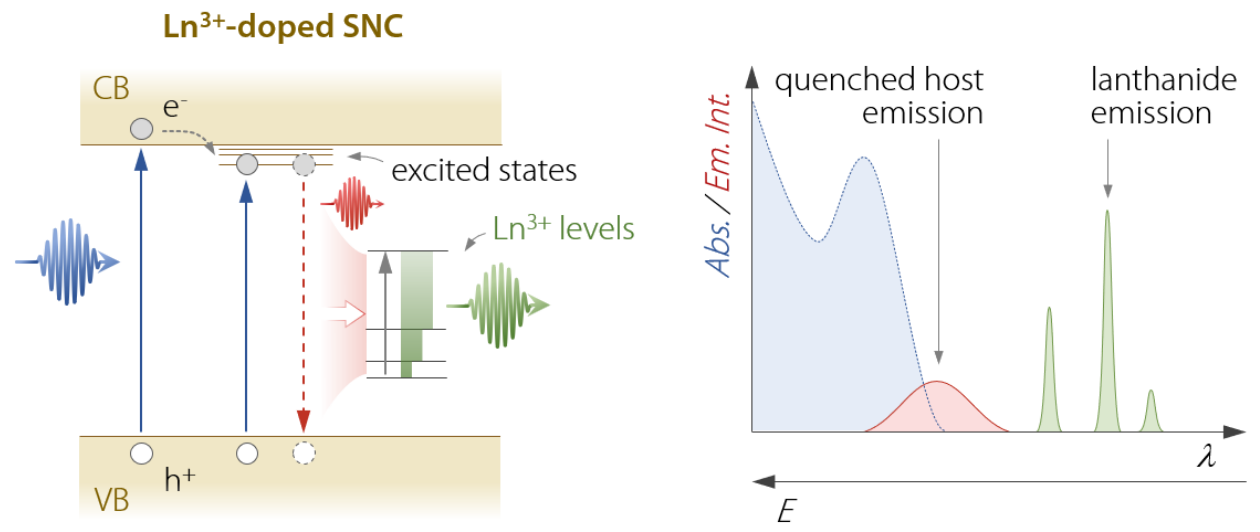

Figure 2. Simplified energetic scheme of a generic LnSNC along with the corresponding expected absorption and emission spectra. After absorption of the excitation light (blue solid arrows), electron and hole recombine (red dashed arrow) transferring the energy to the doped $\mathrm{Ln}^{3+}$ ion (horizontal white arrow), which in turn emits a photon. The term "excited states" here is introduced to refer to a generic intermediate state that can act as a bridge for the population of $\mathrm{Ln}^{3+}$ excited states - these states can be excitonic or defect levels, as well as charge transfer states (vide infra). Thanks to this transfer of absorbed energy to the $\mathrm{Ln}^{3+}$ ion, the emission of the semiconductor moiety results partially (or completely) quenched.

along with the chemical and photo-stability of an all-inorganic system. This strategy has a close relative in the preparation of luminescent $\mathrm{Ln}^{3+}$ complexes. ${ }^{3}$ There, the role of antenna is played by the organic ligands that coordinate the metal center. Such molecular approach has proven very successful for the preparation of brightly emitting $\mathrm{Ln}^{3+}$ complexes. However, some applications require the use of systems that are more resilient to harsh chemical environments than molecular species, and that do not easily undergo photobleaching. In those instances, colloidal LnSNCs are poised to make a huge difference. For instance, in nanomedicine these nanoparticles could represent a new generation of optical contrast agents with unmatched penetration depth within biological tissues and high signal-to-noise ratio, allowing fast readout. In photovoltaics, their light harvesting capabilities, emission brightness, and foreseeable stability to prolonged irradiation would enable achieving record performances in terms of energy conversion and durability.

\section{Designing a lanthanide-doped semiconductor nanocrystal}

\subsection{Chemical considerations}

Let us start breaking down the various aspects of LnSNC design. To begin with, we should consider the chemistry of $\mathrm{Ln}^{3+}$ ions. They are characterized by a 3+ oxidation state, are highly oxophilic, and prefer crystal sites with high coordination number $(\mathrm{CN} \geq 6) .{ }^{79}$ Most semiconductor materials are composed of transition and/or main group metals. The oxidation state of these elements can vary between 1+ and $4+$, they often have a greater affinity towards heavier chalcogens (e.g., $\mathrm{S}^{2-}$ and $\mathrm{Se}^{2-}$ ), and can occupy lattice sites with both low and high $\mathrm{CN}$ depending on the crystal phase of the material. Moreover, one should consider differences in ionic radius, which might prevent a $\mathrm{Ln}^{3+}$ to be incorporated in a substitutional lattice site due to steric hindrance. At first sight, the mismatch between the two families of elements seems irreconcilable. But there are ways around those obstacles.

3.1.1. Cation site and coordination environment. In many classical semiconductors (e.g., CdSe, ZnS, InP, $\mathrm{GaN})$ the metal center sits in tetragonally coordinated sites ( $\mathrm{CN}=4$ - see Figure $\mathbf{3 A}){ }^{80}$ This coordination 
A

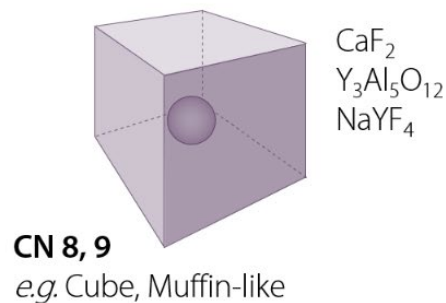

\section{Cation coordination environment}

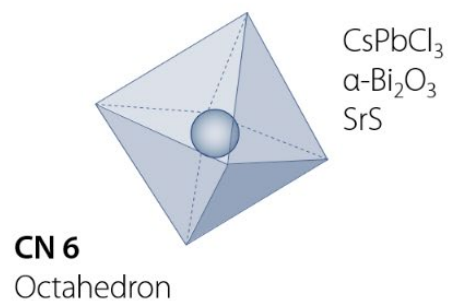

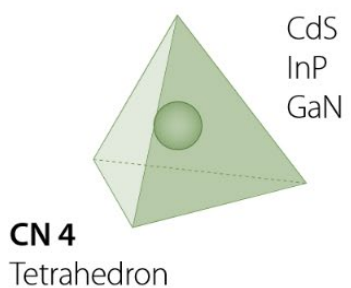

CdS

$\ln P$

$\mathrm{GaN}$
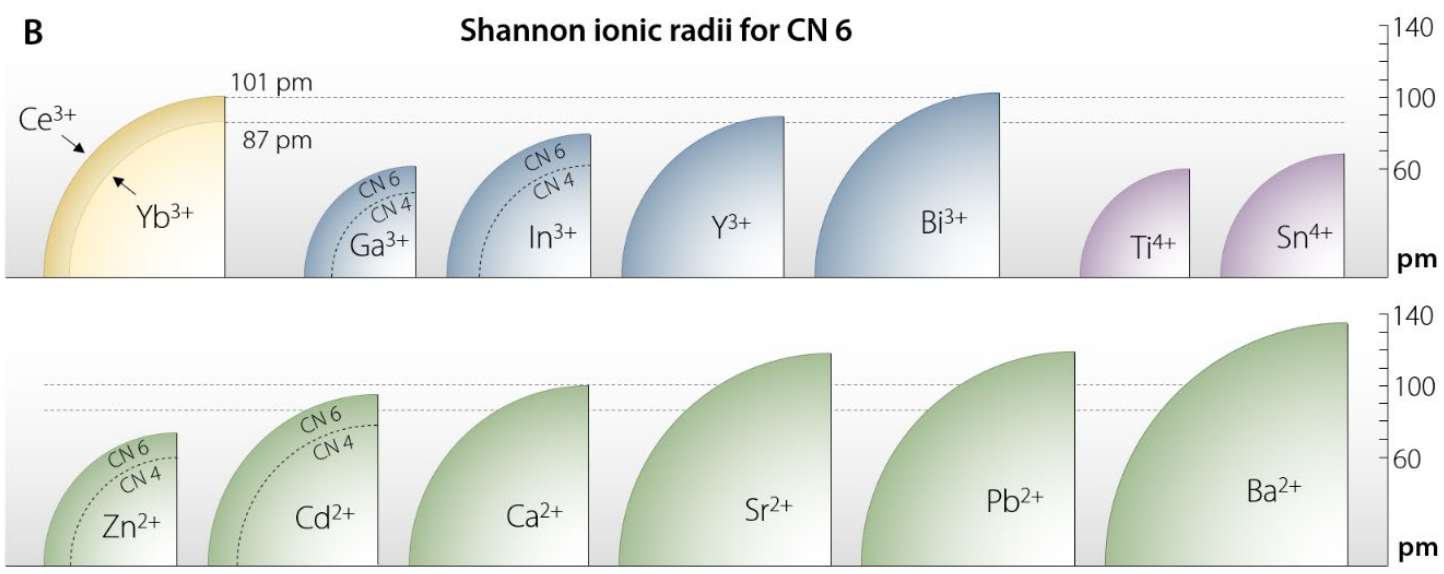

Figure 3. A: Coordination geometries, with relative coordination number $(\mathrm{CN})$, found in hosts of interest for $\mathrm{Ln}^{3+}{ }_{-}$ doping. Only the coordination environment of the cation that can be substituted by a $\operatorname{Ln}^{3+}$ ion is shown. $\mathbf{B}$ : Graphical representation of ionic radii according to Shannon's formula 81,82 for $\mathrm{Ln}^{3+}$ ions and the most relevant cations composing semiconductors grouped by oxidation state. The radius of each element is the one for CN 6 , unless otherwise indicated.

is not the one preferred by $\mathrm{Ln}^{3+}$ ions: because of their hard Lewis acid nature*, $\mathrm{Ln}^{3+}$ are stabilized by a sizeable electron density, which is more easily provided by a larger number of anions. To that end, it is helpful to recall that doping of $\mathrm{Ln}^{3+}$ is easily accomplished in dielectric host materials such as garnets and fluorides, where $\mathrm{CN}=8$ or $9.38,83$ Such high coordination numbers are rarely observed in semiconductors, but cation sites with octahedral coordination $(\mathrm{CN}=6)$ are found in many members of this family of materials. The selection of semiconductors featuring octahedral (or higher- $\mathrm{CN}$ ) cation sites appears to be the fundamental prerequisite for an effective incorporation of $\mathrm{Ln}^{3+}$. In that regard, the outstanding results obtained as of late with halide perovskites strongly corroborate this assumption. . $^{1,55,}$ ${ }^{84}$ In fact, halide perovskites (and alkaline earth sulfides) have octahedral cation sites that can easily accommodate $\mathrm{Ln}^{3+}$ and the incorporation of these ions can be achieved by relying on statistical mixing (see Sections 6.3 and 6.4). Oppositely, the effective incorporation of $\mathrm{Ln}^{3+}$ ions in semiconductors with tetrahedral cation sites often requires high-energy methods, such as ion implantation ${ }^{85,86}$ or annealing at temperatures above $800{ }^{\circ} \mathrm{C}$ to promote ionic diffusion at solid state $e^{87-90}$.

3.1.2. Ionic radius and oxidation state. The choice of a host featuring cation sites with $\mathrm{CN} \geq 6$ is the most important requisite. However, oxidation state and cationic radius also matter. The Shannon ionic radius ${ }^{82}$ of $\mathrm{Ln}^{3+}$ ions in an octahedral site goes from $101\left(\mathrm{Ce}^{3+}\right)$ to $87 \mathrm{pm}\left(\mathrm{Yb}^{3+}\right)$ decreasing along the series due to

\footnotetext{
* A Lewis acid is a species that accepts lone electron pairs from a donor species (Lewis bases). The less (more) the Lewis acid is polarizable, the harder (softer) its acid character is, according to the hard soft acids bases (HSAB) theory. This acidity can be regarded as a measure of how "thirsty" for electrons a species is.
} 
the lanthanide contraction effect. Figure $\mathbf{3 B}$ shows how the ionic radius of other cations typically found in semiconductors varies considerably from element to element. If the cation in the host material is too small, $\mathrm{Ln}^{3+}$ doping might be impeded or disruptive consequences in terms of host integrity are to be expected upon the incorporation of the dopants (due to strain in the lattice). Likewise, an oxidation state mismatch will introduce defects in the material (such as ion vacancies) to guarantee charge compensation - which might also result in loss of crystallinity.

To better understand the interplay between the three parameters discussed above, it is instructive to consider the results obtained for the doping of $\mathrm{Ln}^{3+}$ ions in different host materials. For instance, $\mathrm{Ln}^{3+}$ can be doped easily into the octahedral site of $\beta-\mathrm{Ga}_{2} \mathrm{O}_{3}$ despite the radius mismatch (approx. 30\%) with $\mathrm{Ga}^{3+}$, also owing to the same oxidation state of the two cations. ${ }^{91-94}$ The situation is even more favorable for $\mathrm{Ln}^{3+}$ substitutional doping in cubic (bixbyite-type) $\ln _{2} \mathrm{O}_{3}$, since $\ln ^{3+}$ (which occupies sites with $\mathrm{CN}=6$ ) has an ionic radius very close to the one of $\mathrm{Ln}^{3+}$ ions ( $80 \mathrm{pm}$ ). ${ }^{95-97}$ Instead, incorporation in CdS or CdSe is relatively ineffective. ${ }^{49,98}$ In these materials, $\mathrm{Cd}^{2+}$ occupies tetrahedral sites and, although the radius mismatch is smaller, incorporation of $\mathrm{Ln}^{3+}$ also brings a charge imbalance in the lattice - which requires the appearance of defects for charge compensation. Overall, $\mathrm{Ln}^{3+}$ doping of CdS (or even ZnS) is therefore not energetically favorable..$^{54}$ Another significant example is offered by $\mathrm{Ln}^{3+}$ doping of anatase $\mathrm{TiO}_{2}{ }^{99-101}$ Although therein the cations occupy octahedral sites, a severe radius mismatch between $\mathrm{Ti}^{4+}$ and $\mathrm{Ln}^{3+}$ is observed along with a difference in oxidation state. In fact, the substitution of $\mathrm{Ti}^{4+}$ by $\mathrm{Ln}^{3+}$ takes place only at low doping concentration. Increasing the lanthanide content, the incorporation proceeds through occupation of an octahedral interstitial site that is large enough to accommodate the aliovalent ion. ${ }^{101,102}$ Particularly interesting is the case of halide perovskites - such as $\mathrm{CsPbCl}_{3}$ - a class of materials that has gained incredible attention in the past few years. In $\mathrm{CsPbCl}_{3}, 51,55,84$ the octahedral sites are occupied by $\mathrm{Pb}^{2+}$, which is substantially larger than $\mathrm{Ln}^{3+}$ and features different oxidation state. Nonetheless, this is by far one of the most successful semiconductor host in terms of incorporation efficiency and luminescence properties..$^{51,84,103}$ Aside from the favorable coordination geometry, there a $\mathrm{Ln}^{3+}$ ion finds a cation site that is larger than the one it usually occupies. This situation is clearly less troublesome than "squeezing" the $\mathrm{Ln}^{3+}$ in a cation site that is too small - which is the case in $\mathrm{TiO}_{2}$. The incorporation of $\mathrm{Ln}^{3+}$ in $\mathrm{CsPbCl}_{3}$ is also bound to introduce defects in the structure for charge compensation. As a matter of fact, it was recently hinted that the presence of pre-existing defect in the structure might lead to doping of $\mathrm{CsPbCl}_{3}$ with large amounts of $\mathrm{Ln}^{3+} .{ }^{103} \mathrm{~A}$ conclusive understanding of the structure-property behavior of halide perovskites is far from being achieved, ${ }_{1}^{104}$ but it is now accepted that halide perovskites are exceptionally defect-tolerant in terms of preservation of the optical and structural properties (see Section 6.3)..$^{105}$

In alkaline earth sulfides (AESs) like CaS and SrS, we find a situation similar to the one of Pb-based perovskites: regardless of the charge and radius (in the case of $\mathrm{Sr}^{2+}$ ) mismatch, these materials can be efficiently doped with $\mathrm{Ln}^{3+}{ }^{106-109}$ - although AESs can also stabilize the divalent form of lanthanides, depending on the predisposition of the doped ion to be reduced. To that end, cerium can be introduced as $\mathrm{Ce}^{3+}$ in those hosts, while europium takes the $2+$ oxidation state (see Section 6.4). ${ }^{106,108}$

Among the elements presented in Figure 3B, yttrium represents a special case. It is usually grouped with lanthanides and scandium in the family of rare-earths (REs) due to the similar chemistry featured by these elements. $Y^{3+}$ prefers high-CN cation sites, it has same oxidation state of $\mathrm{Ln}^{3+}$ and comparable ionic radius. Therefore, $\mathrm{Y}^{3+}$-containing compounds are ideal host material for the incorporation of $\mathrm{Ln}^{3+}$ ions.

3.1.3. Divalent europium. Although we are focusing on $\mathrm{Ln}^{3+}$ ions, $\mathrm{Eu}^{2+}$ is a technologically relevant ion, particularly from an optical standpoint and we will discuss its use further throughout this Review. Because of this, it is worth mentioning that also $\mathrm{Eu}^{2+}$ is better accommodated at high-CN sites ${ }^{110}$ and it has an ionic radius of $117 \mathrm{pm}$ for $\mathrm{CN}=6$ (close to the one of $\mathrm{Ce}^{3+}$ of $101 \mathrm{pm}$ ). However, its nominal charge (2+) suggests a more straightforward substitution of divalent cations since no anion or anion 
vacancy should be introduced upon $\mathrm{Eu}^{2+}$ doping in the lattice. As far as chemical precursors are concerned, salts of Eu ${ }^{2+}$ can be employed, ${ }^{111}$ but their poorer solubility compared to $\mathrm{Eu}^{3+}$ salts limits their use in wet-chemistry synthesis at the nanoscale. ${ }^{112}$ Fortunately, the redox potential of the $\mathrm{Eu}^{3+} / \mathrm{Eu}^{2+}$ couple $(-0.36 \mathrm{~V})$ is the smallest in magnitude compared to the other $\mathrm{Ln}^{3+} / \mathrm{Ln}^{2+}$ couples. ${ }^{113}$ As a result, $\mathrm{Eu}^{2+}-$ doped SNCs can be prepared starting from $\mathrm{Ln}^{3+}$ salts if the host material and the reaction environment are carefully chosen (e.g., by the presence of oleyl amine as reducing agent). ${ }^{114}$

3.1.4. Summary. Given the above considerations, in order to maximize the possibility to succeed in doping $\mathrm{Ln}^{3+}$ ions into a semiconductor lattice, one has to i) select a semiconductor material where cationic sites with $\mathrm{CN} \geq 6$ are available, ii) prefer semiconductors where the cation to be substituted has $3+$ oxidation state, iii) aim for a minimal ionic radius mismatch between the dopant and the cation to be substituted - although $\mathrm{Ln}^{3+}$ ions can also substitute larger cations more easily than smaller ones. These observations are valid in general, not only for nanocrystals. Nonetheless, the synthesis at the nanoscale presents more challenges than the preparation of bulk systems due to the impossibility to reach temperatures higher than $300-350^{\circ} \mathrm{C}$ - a limit imposed by the boiling points of commonly used solvents - and the fine balance that has to be achieved over the reactivity of each species during the growth of the material (see Section 4).

\subsection{Physical considerations}

We have provided above the guidelines to select a semiconductor that maximizes the chances of doping $\mathrm{Ln}^{3+}$ ions in its lattice. However, how can we be sure that the host material would effectively transfer the optical energy absorbed to the $\mathrm{Ln}^{3+}$ ion? And will the $\mathrm{Ln}^{3+}$ ion re-emit $100 \%$ of this energy upon receiving it? Being able to answer those questions, or at least foresee possible obstacles preventing those situations to take place, is pivotal. In this section, we analyze the physical aspects that one should consider to ensure proper host-mediated sensitization of $\mathrm{Ln}^{3+}$ photoluminescence in LnSNCs.

3.2.1. Bandgap and interbandgap states. In the design of luminescent $\mathrm{Ln}^{3+}$ complexes, attention has to be paid to the position of the triplet state of the ligand, since generally from that electronic state the energy absorbed by the organic moiety is transferred to the metal center (Figure 4A) ${ }^{3,7,58}$ It should however be noted that the singlet state can substantially contribute to the $\mathrm{Ln}^{3+}$ sensitization too. When the energy difference between the triplet state and the $\mathrm{Ln}^{3+}$ emitting state is too small, the energy can easily jump back and forth between the two states, leading to an overall poor sensitization of the metal emission. When the triplet state is too high in energy, the population of the $\mathrm{Ln}^{3+}$ emitting state can be difficult, since the energy transfer (ET) would require the participation of multiple phonons to bridge the energy mismatch. ${ }^{*}$ It is thus necessary to find the right balance between those two situations. In a first approximation, it is reasonable to assume that in LnSNCs the optical bandgap can be roughly regarded as the equivalent of the triplet state energy (Figure $\mathbf{4 B})^{98}$

The advantage of using a SNC to sensitize the emission of $\mathrm{Ln}^{3+}$ is that one can tune the bandgap upon using the strategies shown in Figure 1C and 1D. As an example, we show in Figure 4B the effect that size tuning has on the relative position between the electronic bands of the semiconductor and $\mathrm{Ln}^{3+}$ energy states. One of the processes through which the sensitization of the doped $\mathrm{Ln}^{3+}$ can take place is via non-radiative energy transfer (ET) from the host (energy donor) to the lanthanide ion (energy acceptor). ${ }^{115}$ For ET to occur, it is evident that the magnitude of the energy released upon recombination of the carriers in the semiconductor should be at least equal to the energy of the emitting state of the $\mathrm{Ln}^{3+}$ ion. In a first approximation, one can assume that the role of the donor is played by the

\footnotetext{
* This second situation is less critical for $\mathrm{Ln}^{3+}$ ions that have energy states above the emitting state. Those higherenergy states can act as energy acceptors and eventually lead to the population of the emitting state via subsequent non-radiative de-excitation steps.
} 
A

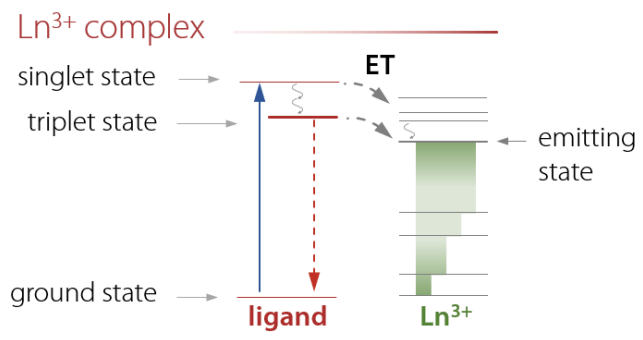

B $\quad L n S N C$

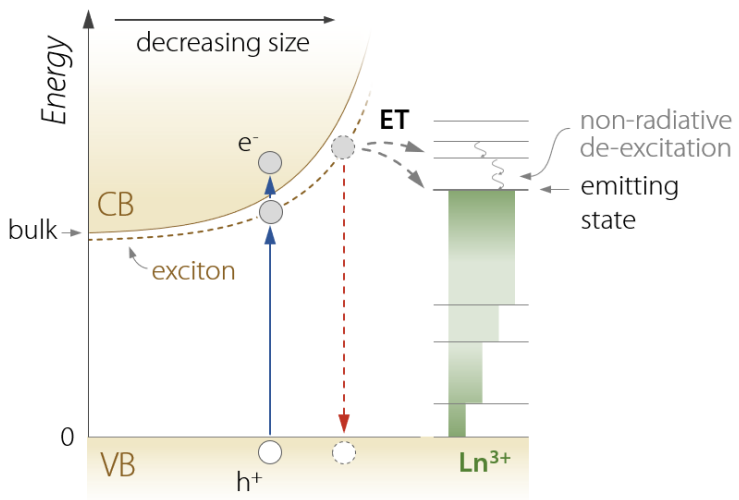

C

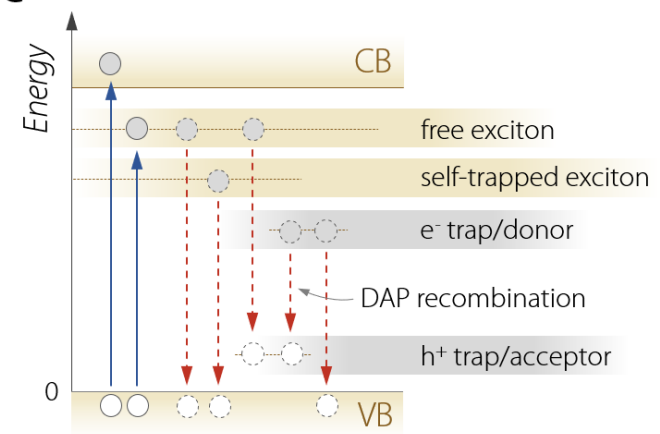

D

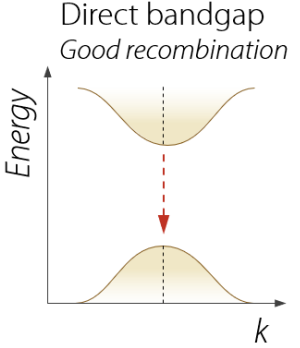

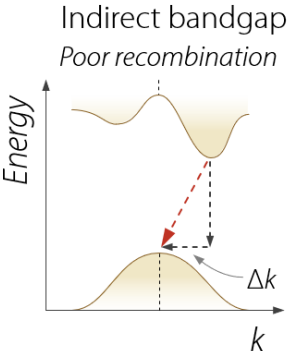

Figure 4. Comparison of the partial energy level scheme of a generic $\mathrm{Ln}^{3+}$ complex $(\mathbf{A})$ and a $L \mathrm{~L}^{3+}$ ion doped in the lattice of a SNC (B) whose bandgap can be tuned with size. Note that, depending on the size of the nanocrystal, the emitting state can be located at an energy higher or lower than the one of the bandgap. Recombination (dashed red arrows) between the charge carriers (i.e., $\mathrm{e}^{-}$and $\mathrm{h}^{+}$) can provide enough energy to populate $\mathrm{Ln}^{3+}$ excited states via energy transfer - ET. When $\mathrm{Ln}^{3+}$ states located at higher energy than the emitting one are populated, non-radiative de-excitation (gray wavy lines) can effectively lead to the population of the emitting state. B: Generic energy level scheme for a SNC where electron and hole trap states (also referred to as donor and acceptor states) are present. Those inter-bandgap states arise from dopant ions, native defects, and/or dopantinduced defects. All the transitions indicated with a dashed red arrow can contribute to the population of doped $\mathrm{Ln}^{3+}$ ions or to the overall luminescence of the nanocrystal. C: graphical representation of direct and indirect bandgap semiconductors. Red dashed lines represent the recombination process. In the case of an indirect bandgap, the recombination occurs with the assistance of other particles (phonon or electron) that absorb excess momentum.

excitonic state populated upon excitation of an electron from the VB to the CB (see Section 2.2 and Figure 1). The exciton state lies below the continuous of levels of the $C B$ at a distance referred to as exciton binding energy, which normally decreases upon increasing the size of the SNC.62,116 To that end, the onset of the absorption spectrum returns the exciton position (optical bandgap), which is the most relevant parameter in this context, since from there the ET could occur. This is the mechanism proposed by Chen and co-workers in $\mathrm{In}_{2} \mathrm{O}_{3}$ SNCs doped with $\mathrm{Eu}^{3+}{ }^{37}$ Therein, like depicted in Figure 4B, recombination of an electron-hole pair in the SNC leads to the resonant ET to Eu ${ }^{3+}$ high-energy excited states, followed by non-radiative relaxation to the emitting state $\left({ }^{5} D_{0}\right)$ and final radiative decay to the lower-energy states.

However, although the bandgap gives a general idea of the possibility to observe host sensitization of $\mathrm{Ln}^{3+}$ emission, several studies have shown that other energy levels induced by impurities or native to the semiconductor play a key role in the ET mechanism (Figure 4C). 50,52,84,98, 117, 118 In fact they might be the most important levels feeding the $\mathrm{Ln}^{3+}$ emitting state. For instance, in polarizable hosts with strong electron-phonon coupling, localized self-trapped excitons can form. ${ }^{64,119}$ The emission of many halide 
perovskites arises precisely from recombinations of said type of excitons. ${ }^{119}$ Bound excitons can form at localized lattice defects/impurities that introduce interbandgap states just above the VB or below the CB. ${ }^{56,64,120}$ The former can act as electron traps and the latter as hole traps - the two are also referred to as donor and acceptor levels, respectively. The recombination can therefore involve trapped and free charge carriers as shown in Figure 3B. One particular case is the so-called donor-acceptor-pair (DAP) recombination that takes place between a trapped electron and a trapped hole. This wealth of transitions can open several ET channels for the population of $\mathrm{Ln}^{3+}$ excited states. For instance, in $\mathrm{Eu}^{3+}-$ doped $\mathrm{ZnO}$ the involvement of defect states seems to be the mechanism for the sensitization of the lanthanide emission. ${ }^{121,}{ }^{122}$ Nonetheless, it is expected that the availability of defect states could also heavily contribute to the depopulation of the $\mathrm{Ln}^{3+}$ emitting level. Therefore, a thorough, ad hoc research about possible native interbandgap states present in the host material is necessary. For instance, $\mathrm{CulnS}_{2}$ is known to feature DAP-related luminescence in its bulk form, ${ }^{123}$ while VB-to-Cu+2 ${ }^{+/ 2+}$ hole trap states appear to be the electron transitions responsible for the luminescence in nanocrystals ${ }^{124,125}$. Importantly, doping with $\mathrm{Ln}^{3+}$ ions can induce formation of defects too, which can in turn act as effective intermediate states for the sensitization of the dopant ion. $\mathrm{CsPbCl}_{3}$ is a good example in this context: the incorporation of rare-earth ions creates defect complexes of the type $\mathrm{RE}^{3+}-\mathrm{VPb}_{\mathrm{Pb}}-\mathrm{RE}^{3+}$ (where $\mathrm{V}_{\mathrm{Pb}}$ is a $\mathrm{Pb}^{2+}$ vacancy), which support quantum cutting emission of $\mathrm{Yb}^{3+}$-doped $\mathrm{CsPbCl}_{3} \cdot{ }^{84}$ It has also been observed that in AESs the addition of $\mathrm{Pr}^{3+}$ promotes the formation of deep electron trap states within the bandgap: a situation that enables delayed photoluminescence in the visible following de-trapping of the electrons under NIR excitation (see Section 6.4). ${ }^{106,126}$

One aspect that we have so far disregarded is the distinction between direct and indirect bandgap semiconductors (Figure 4D ). ${ }^{*}$ When the VB maximum and the CB minimum are both located at the same crystal momentum ( $k$ ) of the Brillouin zone, the material has a direct bandgap. This alignment affords an easy recombination of excited electron and hole. ${ }^{127}$ In indirect bandgap semiconductors, this condition is not realized and the carriers recombination can only occur if the excess momentum $(\Delta k)$ of the electron is transferred to the lattice (in the form of phonons) or to an electron (Auger recombination). ${ }^{128,} 129$ Direct semiconductors (e.g., GaN, ZnS) are preferentially used in light-emitting devices and lasers because the recombination events are statistically more probable. Indirect semiconductors (e.g., anatase $\mathrm{TiO}_{2}, \mathrm{c}$-Si) are often used in photovoltaic devices or whenever charge transportation throughout the material is preferred over recombination. In the literature we can observe sensitization of $\mathrm{Ln}^{3+}$ emission using both types of semiconductors (e.g., $\mathrm{TiO}_{2}{ }^{130}$ and $\mathrm{In}_{2} \mathrm{O}_{3}{ }^{97}$ with indirect and direct bandgap respectively) and it is difficult to gather information that allows proper comparison of the performance in the two situations. It is reasonable to think that direct bandgap semiconductors should favor population of $\mathrm{Ln}^{3+}$ excited states that occurs from the recombination of free excitons, given the higher exciton recombination rate in these materials. Instead, in indirect bandgap semiconductors, charge carriers can travel over a longer distance (larger mean free path) thus increasing the probability of them encountering trap states. ${ }^{64}$ This process could promote sensitization of the $\mathrm{Ln}^{3+}$ emission through excitons bound to impurity-related levels. However, indirect bandgap materials suffer from intrinsic lower absorption capability, which also more heavily depends on the temperature. More studies are required to better understand the possible role of this parameter.

3.2.2. Charge transfer. Another effect that should be taken into account when designing photoluminescent LnSNCs is charge transfer (CT). This phenomenon is often observed in materials doped with $\mathrm{Eu}^{3+}$ and $\mathrm{Yb}^{3+}{ }^{331}, 132$ We can understand why is that considering a model developed by Dorenbos, which allows determining the relative position between the energy states of lanthanide ions

\footnotetext{
* Preliminary information about the nature of the bandgap can be inferred from the absorption spectrum of a material. Steeper absorption onset generally points towards a direct bandgap, while a more gradual onset is indicative of an indirect bandgap.
} 
and the host material of concern. ${ }^{133,134}$ The detailed explanation of this approach lies beyond the scope of this Review and the interested reader is referred to the large body of literature on the subject. ${ }^{135}$ However, one can obtain a rough idea about the relative position of lanthanide $4 \mathrm{f}$ energy levels and host electron bands - and hence about the possibility of CT phenomena to occur - through the approximation used by Mukherjee et al. ${ }^{54}$ This entails the estimation of the ground state position of divalent europium $\left(\mathrm{Eu}^{2+}\right)$ using Jörgensen's empirical formula $E^{C T}(\mathrm{Eu})=3.72\left(\chi_{o p t}(x)-\right.$ $\left.\chi_{\text {opt }}(\mathrm{Eu})\right) \mathrm{eV}$. $^{136}$ There, $\chi_{\text {opt }}(\mathrm{Eu})$ and $\chi_{\text {opt }}(x)$ are the optical electronegativity of europium and the anion that transfers the charge to the metal ion, respectively. Taking $\chi_{\text {opt }}(\mathrm{Eu})=2$ and the electronegativity of the anion from the values corrected by Allred, ${ }^{137}$ one gets the average $E^{C T}$ (Eu) for each class of host material (Figure $\mathbf{5 A}$ ). ${ }^{138^{*}}$ This parameter returns the position of the $\mathrm{Eu}^{2+}$ ground state above the $\mathrm{VB}$, since $E^{C T}(\mathrm{Eu})$ represents the energy necessary to transfer an electron from the host material to $\mathrm{Eu}^{3+}$. Experimentally, this phenomenon translates to the presence of an absorption band (CT band), whose position varies depending on the $\mathrm{Ln}^{3+}$ doped in the host material. Interestingly, Figure $\mathbf{5 A}$ shows that more polarizable hosts induce a CT at lower energy. Mukerjee et al. then considered an energy difference between $\mathrm{Eu}^{2+}$ and $\mathrm{Eu}^{3+}$ ground states, $U(\mathrm{Eu})$, of $5.7 \mathrm{eV}$. This is another approximation, since $U(E u)$ scales with the bandgap of the material. ${ }^{138,139}$ For semiconductors, an error of at least $\pm 0.2 \mathrm{eV}$ around $5.7 \mathrm{eV}$ should be accounted.

The relative position of the ground states of divalent and trivalent lanthanides follows a general trend, which can be used to obtain a scheme like the one reported in Figure 5B for lanthanide-doped ZnS. To build that scheme, we used tabulated values reported by Dorenbos for more polarizable hosts ${ }^{138},{ }^{\dagger}$ obtaining a good agreement with the scheme reported in Ref. ${ }^{54}$.
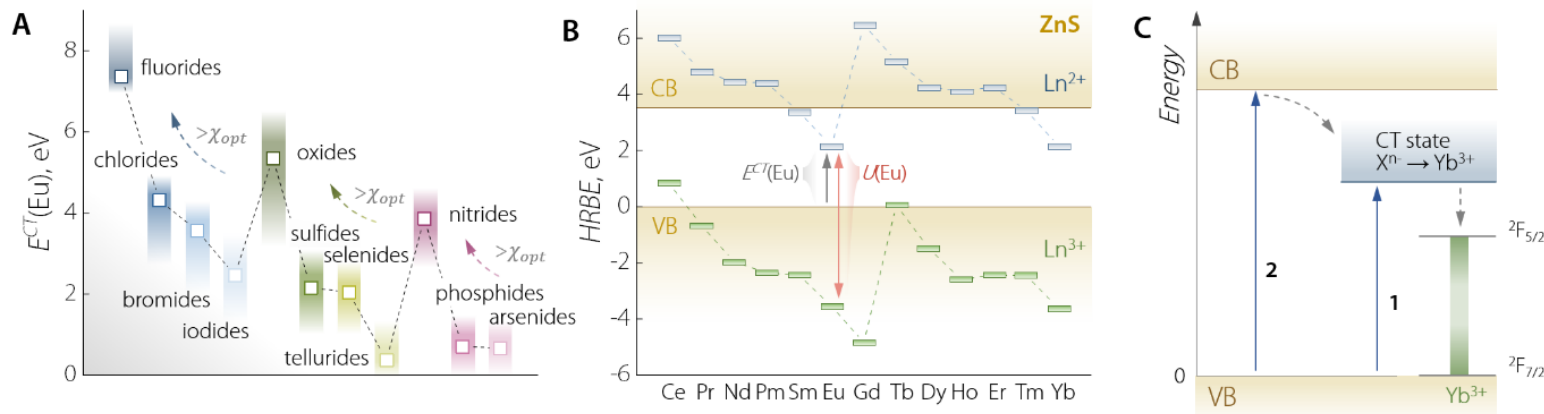

Figure 5. Charge transfer phenomena in lanthanide-doped materials. A: Trend of europium charge transfer band position $E^{C T}(E u)$ in different semiconductors grouped by anion. Squares are points obtained from the approximated expression discussed in the main text, while gradient bands highlight the dispersion of the experimental points obtained from the literature. Adapted with permission from Ref. ${ }^{138}$. Copyright 2005 Elsevier. B: Host-referred binding energy (HRBE) diagram obtained for the system ZnS: $\mathrm{Ln}^{2+/ 3+}$. C: Sensitization mechanisms of $\mathrm{Yb}^{3+}$ involving direct excitation in the $C T$ band (1) and its indirect population upon excitation in the CB of the host material (2). $X^{n-}$ is a generic anion in the semiconductor lattice (with charge $\mathrm{n}$-) that transfers the electron to $\mathrm{Yb}^{3+}$.

\footnotetext{
* The approximation done here does not consider the length of the bond between the lanthanide and the anions, nor the number of anions surrounding the metal. Clearly, more accurate ways to determine the effective polarizability exist, but their description lies beyond the scope of the present Review.

${ }^{+}$These values are obtained considering that in more polarizable hosts the $5 \mathrm{~d}$ levels are flat. Deviations are observed for less polarizable hosts, particularly for the elements with more than half-filled $4 \mathrm{f}$ shell (i.e., those following $\mathrm{Gd}^{3+}$ in the series).
} 
The reasonable positioning of $\mathrm{Eu}^{2+}$ ground state $(2.16 \mathrm{eV})$ is corroborated by the experimental observation of a broad excitation band ascribed to $C T$ (centered approximately at $2.43 \mathrm{eV}$ ) upon monitoring the emission of Eu ${ }^{3+}$-doped $\mathrm{ZnS}$, which was not observed in the case of $\mathrm{Tb}^{3+}$ doping. ${ }^{54}$ From Figure $\mathbf{5 B}$, one can conclude that a CT band should be observed also in the excitation spectrum of $\mathrm{Yb}^{3+}$ doped ZnS. In general, given the lower position of their $\mathrm{Ln}^{2+}$ ground state, samarium, europium, thulium, and ytterbium are the lanthanides that could profit the most from CT sensitization in more polarizable hosts. To that end, two types of CT-mediated sensitization mechanisms have been proposed (Figure 5C): i) direct excitation in the $\mathrm{CT}$ band (e.g., in $\left.\mathrm{CaSO}_{4}: \mathrm{Eu}^{3+}\right)_{1}^{131,140}$ or ii) excitation in the SNC CB followed by transfer to the $\mathrm{CT}$ band (e.g., in InP:Yb $\left.{ }^{3+}\right)^{50,141}$

Lastly, we should point out that the CT band of europium can span a range of more than $2 \mathrm{eV}$ for a given class of materials (Figure $\mathbf{5 A}$ ). ${ }^{138}$ When designing a LnSNC, even a couple hundreds of millielectronvolts might make the difference between bright or quenched luminescence. Hence, if CT phenomena are of interest, one should obtain experimental data from the literature, where usually studies on bulk materials are found. ${ }^{142-146}$ More studies conducted on nanocrystals at low temperature are desirable to investigate the influence of quantum effects on CT phenomena and the possibility to harness them for $\mathrm{Ln}^{3+}$ sensitization.

3.2.3. Cation site symmetry. As discussed above, the availability of a high-CN cation site is a fundamental prerequisite for facile incorporation of $\mathrm{Ln}^{3+}$ ions in the lattice. But there is more to be considered in that regard. As a matter of fact, the symmetry of the site occupied by the $\mathrm{Ln}^{3+}$ ion has a non-negligible impact on the optical properties of the system. ${ }^{147}$ First, a centrosymmetric site (i.e., featuring an inversion center as symmetry element) is most detrimental to $\mathrm{Ln}^{3+}$ emission, since it greatly reduces the probability of breaking the Laporte rule (vide supra). It seems unfortunate that perfect octahedral - and many cubic sites suitable for $\mathrm{Ln}^{3+}$ doping are characterized by this element of symmetry. However, commonly the coordination polyhedron is distorted, resulting in a lower local symmetry. That is the case for $\mathrm{Eu}^{3+}$-doped $\beta-\mathrm{Ga}_{2} \mathrm{O}_{3}$, where the dopant ion is incorporated in the octahedral sites of the lattice. ${ }^{94} \mathrm{~A}$ perfect octahedron has local symmetry $\mathrm{O}_{h}$ (a centrosymmetric point group), but $\mathrm{Ga}^{3+}$ is found at a cation site with symmetry $C_{s}$. Not only this latter point group does not have an inversion center, but it also features merely two elements of symmetry versus the 48 of a perfect octahedron. On top of that, when entering the host lattice, a $\mathrm{Ln}^{3+}$ ion is bound to bring about further distortions due to differences in the ionic radius. Therefore, although some crystal sites might seem unsuitable from a spectroscopic standpoint, emission from $\mathrm{Ln}^{3+}$ ions doped at those relatively symmetric sites is still observable.

3.2.4. Use of lanthanides with $4 f-5 d$ transitions $\left(\mathrm{Ce}^{3+}\right.$ and $\left.\mathrm{Eu}^{2+}\right)$. Although in this Review emphasis is put on $\mathrm{Ln}^{3+}$ featuring parity-forbidden $4 \mathrm{f}-4 \mathrm{f}$ transitions, there are some specific lanthanide ions whose optical properties are dominated by parity-allowed $4 f-5 \mathrm{~d}$ transition. ${ }^{148}$ Due to their frequent use in lanthanideactivated phosphors, a brief mention to these species is due. The most used lanthanides featuring $4 \mathrm{f}-5 \mathrm{~d}$ transitions found in nanophosphors are $\mathrm{Ce}^{3+}$ and $\mathrm{Eu}^{2+}$ (Figure 6). Tb ${ }^{3+}$ also features $4 \mathrm{f}-5 \mathrm{~d}$ transitions, but those are generally found at relatively high energies and, as such, less exploited. ${ }^{149}$ Other common divalent lanthanide ions featuring those allowed transitions are $\mathrm{Sm}^{2+}, \mathrm{Yb}^{2+}$ and $\mathrm{Tm}^{2+} .{ }^{113}$ However, the stabilization of these divalent ions is more difficult to achieve, particularly using methods for the synthesis of colloidal nanoparticles where the harsh conditions necessary to access the 2+ oxidation state are hardly generated..$^{113}$ This consideration is less critical in the case of Eu ${ }^{2+}$. As abovementioned, the reduced redox potential of the $\mathrm{Eu}^{3+} / \mathrm{Eu}^{2+}$ couple allows preparing $\mathrm{Eu}^{2+}$ solutions even starting from $\mathrm{Eu}^{3+}$ salts. The permanence of the divalent oxidation state when the ion is doped in a crystalline host can be inferred using the approach developed by Dorenbos (Section 3.2.2) and knowledge of the Fermi level of the compound. ${ }^{150}$ 

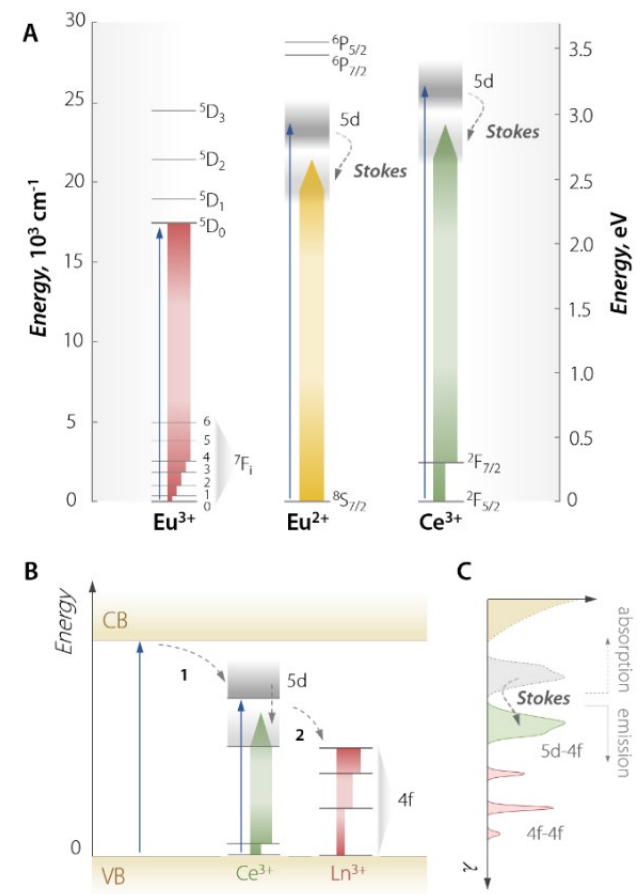

Figure 6. A: Partial energy level scheme for the two prominent lanthanide ions whose 4f-5d transitions are harnessed for luminescence purposes: $\mathrm{Ce}^{3+}$ and $\mathrm{Eu}^{2+}$. For the sake of comparison, the partial energy level scheme of $\mathrm{Eu}^{3+}$ is also shown. The position of $5 \mathrm{~d}$ states is dependent on the host material in which the ions are doped, and so are absorption (blue arrows) and emission (gradient-filled rectangles) in $\mathrm{Ce}^{3+}$ and $\mathrm{Eu}^{2+}$. B: Energy transfer mechanisms that can occur in a SNC doped with $\mathrm{Ce}^{3+}$ and another $\mathrm{Ln}^{3+}$ featuring $4 \mathrm{f}-4 \mathrm{f}$ transitions. Albeit host sensitization of $\mathrm{Ce}^{3+}$ can take place (1), usually direct excitation within the $5 \mathrm{~d}$ states of $\mathrm{Ce}^{3+}$ is accomplished. The absorbed energy can be either emitted by $\mathrm{Ce}^{3+}$ or transferred to a nearby $\mathrm{Ln}^{3+}$ (2). C: Representative absorption/emission spectrum of the SNC whose energy levels are depicted in B. $4 f-5 d$ absorption and $5 d-4 f$ emission of $\mathrm{Ce}^{3+}$ are separated by an energy referred to as Stokes shift. This shift arises from the relaxation of $5 \mathrm{~d}$ states when $4 \mathrm{f}$ electrons are excited from the ground state to the $5 \mathrm{~d}$ states. Indeed, this excitation is accompanied by a change of the configurational coordinates of the system, which results in the lowering of the $5 \mathrm{~d}$ states from where the radiative relaxation occurs.

Simply put, if the ground state of $\mathrm{Ln}^{2+}$ is found below the Fermi level, the lanthanide tends to enter the lattice in its divalent state. ${ }^{150}$ The subject of the stability of divalent lanthanides is complex and its indepth discussion lies beyond the scope of this Review.

$\mathrm{Ce}^{3+}$ - and $\mathrm{Eu}^{2+}$-activated phosphors usually rely on the good absorption properties of these ions, ${ }^{151}$ which are indeed often used as sensitizers of other $\mathrm{Ln}^{3+}$ or luminescent transition metals..$^{38,41,152,153}$ Therefore, albeit host-to-lanthanide ET can occur (see section 6.4), it is generally not the leveraged mechanism to promote bright emission. It is also useful to underscore that the sensitiveness of $5 \mathrm{~d}$ levels on the chemical environment enables fine-tuning of the optical properties of phosphors based on $\mathrm{Ce}^{3+}$ and $\mathrm{Eu}^{2+}$. In their recent review, Qin et al. comprehensively present theoretical and experimental aspects of phosphors based on $4 \mathrm{f}-5 \mathrm{~d}$ transitions. ${ }^{148}$ For instance, upon incorporation of Eu ${ }^{2+}$ in a crystal lattice, its $5 d$ levels are lowered in energy (red-shifted) due to a combination of effects. In first approximation, the magnitude of this red-shift is proportional to the crystal field strength, which follows the trend $\mathrm{F}^{-}<\mathrm{Cl}^{-}<$ $\mathrm{Br}^{-}<\mathrm{I}^{-}<\mathrm{O}^{-}<\mathrm{S}^{-}$. As a result, a broad UV-to-NIR emission range can be covered with different host materials doped with $\mathrm{Eu}^{2+154}$ or $\mathrm{Ce}^{3+152,155}$.

3.2.5. Summary. From a physical point of view, the preparation of a brightly emitting LnSNC requires attention to several details of the dopant-host pair. To that end, the bandgap of the material should be 
larger than the energy of the emitting state of the $\mathrm{Ln}^{3+}$ ion. Semiconductors with narrower bulk bandgaps could be employed too, since the distance between the electronic bands can be further tuned using the strategies outlined earlier (Figure 1C and D). In the host-to-Ln ${ }^{3+}$ energy transfer process, one has also to consider the involvement of interbandgap states, which can be native of the material or induced by incorporation of dopant ion(s). Direct and indirect bandgaps materials both proved to be suitable sensitizers for the luminescence of $\mathrm{Ln}^{3+}$ and probably the effect of this parameter mainly depends on the sensitization mechanism occurring in the material. Finally, a lower local symmetry of the cation site is expected to increase the emission efficiency of $\mathrm{Ln}^{3+}$. However, the incorporation of the dopant itself induces distortions in the coordination environment that conceivably diminish the symmetry. Similar considerations can be extended to $\mathrm{Ce}^{3+}$ and $\mathrm{Eu}^{2+}$, featuring $4 \mathrm{f}-5 \mathrm{~d}$ transitions. However, the allowed nature of the transitions of these ions makes host sensitization a less explored route. Indeed, one usually relies on direct absorption of the light from these ions to obtain their bright broadband emission and/or sensitization of the emission of $\mathrm{Ln}^{3+}$ with $4 \mathrm{f}-4 \mathrm{f}$ transitions.

\subsection{Additional observations on the design}

In Figure 7, we report the partial energy level scheme of the more relevant trivalent lanthanide ions for photoluminescence applications, ${ }^{156}$ along with selected semiconductor host materials (save $\mathrm{Y}_{2} \mathrm{O}_{3}$ ). There, the chief properties of the host material are reported (available cation site geometry and bulk bandgap) as well as emission ranges of the $\mathrm{Ln}^{3+}$ ions (VIS - visible: 300-750 nm; NIR - near infrared: 7502000 nm; MIR - mid infrared: 2000-5000 nm). This is far from being a comprehensive picture, but it allows us to make some more observations on the design of LnSNCs.

Firstly, we should be aware of different properties featured by different polymorphs of the same material, both in terms of optical features and structure. For instance, the bandgap of a- and $\beta-\mathrm{Bi}_{2} \mathrm{O}_{3}$ differs substantially, by approximately $0.3 \mathrm{eV} .{ }^{157}$ In the case of $a-$ and $\beta-\mathrm{Ga}_{2} \mathrm{O}_{3}$, the latter has both tetragonal and octahedral sites, while the former features only octahedral sites. ${ }^{158}$ This is an important consideration since, as previously discussed, most of the classical binary semiconductors have only tetragonal cation sites and their doping with $\mathrm{Ln}^{3+}$ was achieved in their bulk form via highenergy/temperature techniques. ${ }^{159-161}$ However, their nano counterparts often showed poor $\mathrm{Ln}^{3+}$ sensitized emission ${ }^{54,98}$ and the dopant was largely found on the surface of the nanocrystals rather than incorporated in the core. ${ }^{88,98,162,163}$

AESs such as CaS and SrS are included in the figure, but often therein $\mathrm{Ln}^{3+}$ sensitization relies on $\mathrm{Ce}^{3+}-\mathrm{Ln}^{3+}$ co-doping. ${ }^{109} \mathrm{In}$ fact, although host-sensitized emission is observed, efficient light absorption is achieved via the involvement of allowed transitions localized at the doped ions (i.e., $4 f-5 d$ transitions of $\mathrm{Ln}^{2+/ 3+}$ ions), which require lower-energy excitation light. ${ }^{109}$ Another point that has to be kept in mind using AESs is that europium is stabilized to its divalent oxidation state in these materials. ${ }^{107,} 164$ The versatility of AESs from an optical standpoint is further corroborated by the possibility to exploit electron trapping phenomena in $\mathrm{Ln}^{3+}$-doped AESs, which has been harnessed to generate visible emission under NIR excitation. ${ }^{106,108}$

Binary semiconductor nanocrystals are easily produced and most of the explored systems for the sensitized emission of $\mathrm{Ln}^{3+}$ belong to this family of compounds. However, this leaves out a good portion of possibly better candidates for the preparation of colloidal LnSNCs: ternary (or more in general multinary) semiconductors. In that vein, throughout this Review we have summoned halide perovskites several times; yet, there are other foreseeable suitable materials. Take for example $\mathrm{CaGa}_{2} \mathrm{~S}_{4}$. In this ternary sulfide, $\mathrm{Ga}^{3+}$ is coordinated tetrahedrally to four sulfur anions, but $\mathrm{Ca}^{2+}$ sits in three 8-coordinate sites. This site geometry, along with the minimal radius mismatch between $\mathrm{Ca}^{2+}$ and $\mathrm{Ln}^{3+}$ (1.12 vs 1.14-0.99 pm with for $\mathrm{CN}=8$ ), makes it possible to dope the latter ions in the structure. ${ }^{165}$ The synthesis of ternary 


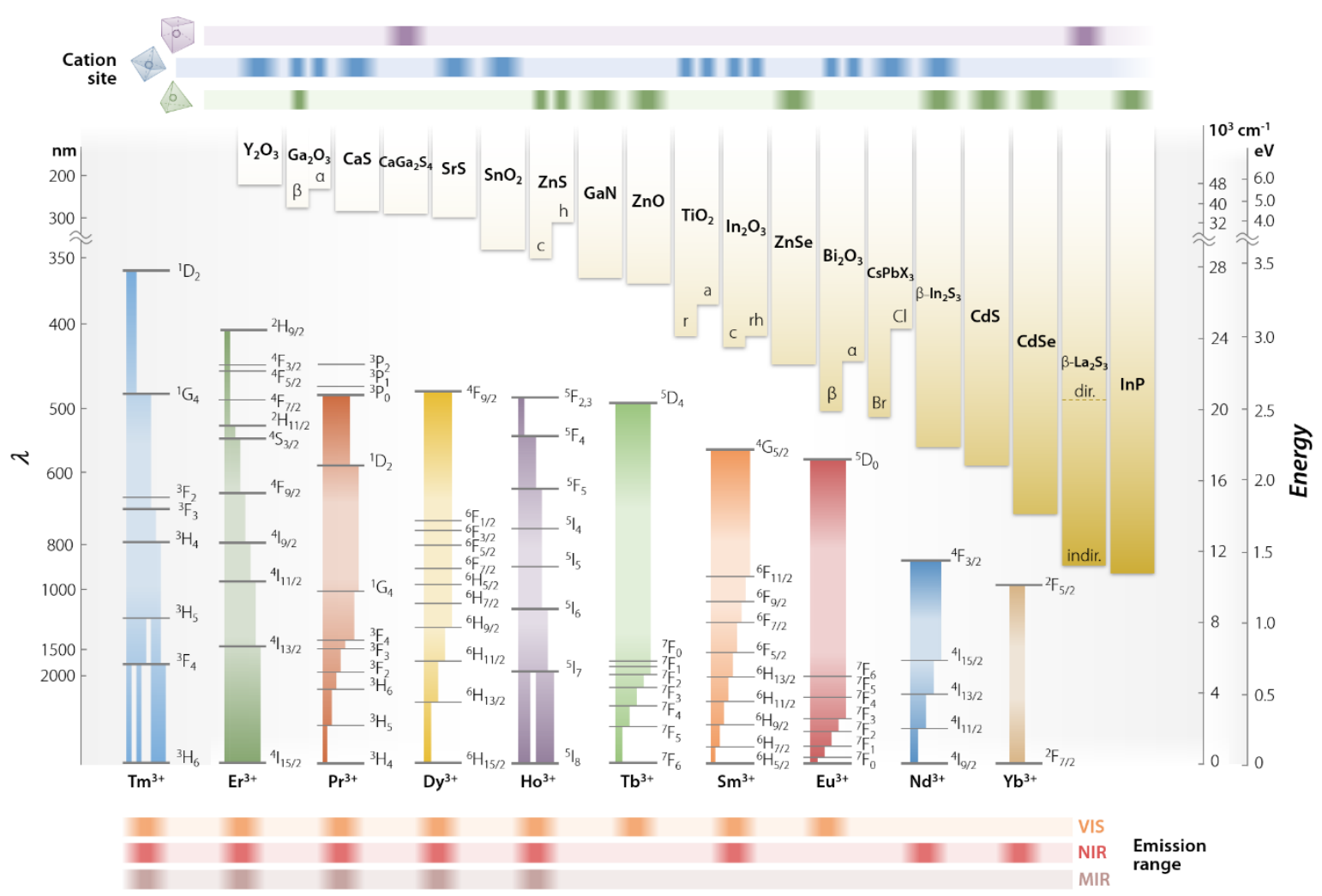

Figure 7. Partial energy level scheme of the most relevant $\mathrm{Ln}^{3+}$ in the preparation of LnSNCs, along with selected semiconductor host materials (except for $\mathrm{Y}_{2} \mathrm{O}_{3}$, which is an insulator). On the top part, available cation site geometries are indicated (green - tetrahedral; blue - octahedral; violet - CN > 6). At the bottom, the emission ranges that each $\mathrm{Ln}^{3+}$ can afford are highlighted. $\mathrm{h}$ - hexagonal, $\mathrm{c}$ - cubic, rh - rhombohedral, For TiO $\mathrm{T}_{2} \mathrm{a}$ - anatase, $r$ - rutile. In $\mathrm{CsPbX}_{3}, \mathrm{X}$ is the halogen (Cl or $\mathrm{Br}$ ).

semiconductor at the nanoscale requires some more fine tuning of the reaction conditions than in the case of binary materials. However, strategies have been developed for the preparation of multinary semiconductors ${ }^{166-168}$ and further research in this direction is expected to prove particularly fruitful in the quest for LnSNCS.

One avenue seems to have been relatively disregarded in the past years for the preparation of LnSNCs exhibiting bright photoluminescence. Some of the hosts that give the best performance in terms of $\mathrm{Ln}^{3+}$ doping are based on $\mathrm{Y}^{3+}$ - see for instance $\mathrm{NaYF}_{4}, \mathrm{YF}_{3}, \mathrm{Y}_{3} \mathrm{Al}_{5} \mathrm{O}_{12}(\mathrm{YAG}){ }^{38,169-172}$ This comes as no surprise, since all the chemistry-related considerations outlined above are respected in those instances (high-CN sites, along with ionic radius and oxidation state match). We suggest that rare-earthbased semiconductors offer the ideal canvas for doping $\mathrm{Ln}^{3+}$ ions. As an example, in Figure $\mathbf{7}$ we report $\beta-\mathrm{La}_{2} \mathrm{~S}_{3}$, which has been used in its bulk form to sensitize the emission of $\mathrm{Nd}^{3+} .{ }^{173}$ In the same study also a-Gd ${ }_{2} S_{3}$ and $y-Y_{2} S_{3}$ were tested, suggesting that binary rare-earth sulfides are attractive candidates for the synthesis of LnSNCS.

We should push these considerations one step further, following the recent trend of investigating ternary nanomaterials. For instance, a close relative of the above-mentioned $\mathrm{CaGa}_{2} \mathrm{~S}_{4}$ is $\mathrm{CaY}_{2} \mathrm{~S}_{4}$, where $\mathrm{Ca}^{2+}$ is 7-coordinate and $\mathrm{Y}^{3+} 6$-coordinate. ${ }^{174}$ Clearly, multinary chalcogenides nanocrystals containing $\mathrm{Y}^{3+}$ or $\mathrm{S}^{3+}$ represent an alluring class of hosts for the preparation of LnSNCS. Thus, methods to prepare rareearth-containing heterometallic SNCs should be investigated more extensively and we envisage that these materials could yield thrilling results. 
One final point shall be made regarding LnSNC absorption/emission range targeted. Most of the research effort has been put so far in the preparation of systems emitting in the visible. $\mathrm{Eu}^{3+}$ and $\mathrm{Tb}^{3+}$ are the usual suspects to that end, although $\mathrm{Sm}^{3+}, \mathrm{Pr}^{3+}$ and $\mathrm{Dy}^{3+}$ are also relevant ions. Eu ${ }^{3+}$ in particular allows to conduct site-selective spectroscopic studies - which provide information regarding the site(s) occupied by the $\mathrm{Ln}^{3+}$ in the lattice (vide infra) - as well as to investigate possible CT phenomena in the material. As of late, the investigation around NIR-emitting materials has experienced a surge, both targeting applications in photovoltaic devices ${ }^{175,176}$ and for biomedical applications $s^{67,}{ }^{177-179}$. The real challenge lies in the development of LnSNCs whose absorption is pushed as much as possible toward the NIR and that also emit in the same long-wavelength range - desirable features particularly targeting nanosystems for biomedical applications, such as fluorescence imaging. This task requires to carefully control the semiconductor bandgap so to push the absorption at lower frequencies while ensuring the possibility of host-to-Ln ${ }^{3+}$ ET (see Section 3.2.1). Moreover, deep $L n^{3+}$ NIR emissions are considerably susceptible to non-radiative quenching, and thus semiconductor hosts based on heavier chalcogens ( $\mathrm{S}$, $\mathrm{Se}, \mathrm{Te}$ ) should perform better than oxides. This consideration stems from observations on $\mathrm{Ln}^{3+}$ complexes featuring Ln-O or Ln-S bonds; indeed, it was verified that the vibrational energy of sulfurcontaining species was much lower than the oxygen-containing counterpart, thus enabling the achievement of higher quantum yields in the NIR. ${ }^{180}$

\section{Synthesis strategies}

Doping of SNC was mainly investigated for transition and main group metals, particularly $\mathrm{Mn}^{181,182}$ $\mathrm{Cu}^{183,184} \mathrm{Ag}^{185} \mathrm{In}^{186,187}$ and $\mathrm{Al}^{188,189}$. As we detail below, the experience gained with those ions has been extended also to $\mathrm{Ln}^{3+}$, implementing the necessary adjustments to account for the different chemistry of lanthanides and transition metals. There are several excellent review papers on the topic of doped SNC syntesis. ${ }^{190-192}$ The group of Gamelin is one of the most prolific in the field and the interested reader is encouraged to an in-depth reading of some of their works. ${ }^{50,107,182,193}$ Because of the existence of several dedicated Reviews on the subject, we only provide a general outline of the approaches developed to prepare doped SNCs with some ad hoc considerations for the case of colloidal LnSNCs.

\subsection{General considerations}

Bulk $\mathrm{Ln}^{3+}$-doped semiconductors have been prepared using techniques such as single crystal growth $^{161,194}$ and solid state reaction in controlled atmosphere ${ }^{195,}{ }^{196}$. Those approaches entail high temperatures, which energetically favor the introduction of dopant ions into the host lattice (thermodynamic control). However, here we are interested in the preparation of colloidal nanocrystals, which is more challenging. The lower temperature and, in general, milder conditions that characterize wet chemical methods yield doping efficiencies mainly determined by kinetic factors. ${ }^{188,190,197}$

At the nanoscale, most of the material's properties are governed by surface effects due to the high surface-to-volume ratio exhibited by nanocrystals (Figure $\mathbf{8 A}$ ). For the sake of discussion, it is useful to visualize the nanocrystal as composed of two different environments: the surface and the core (Figure $\mathbf{8 B}$ ). In terms of crystal lattice, the core of the nanocrystal more closely resembles the bulk material. Still, defects are found therein - also owing to the kinetic control of the nanocrystal growth which can "freeze" the lattice in situations of non-thermodynamic equilibrium. Presence of defects can sometimes boost the rate of phenomena like cation exchange and favor the incorporation of aliovalent species. ${ }^{103}$ The surface features more marked deviation from bulk stoichiometry (it is generally richer in cations), a higher concentration of defects (such as dangling bonds and vacancies), and it provides anchoring points for stabilizing molecules, as well as solvent molecules responsible for most of the luminescence quenching (see Section 4.3). Because of this, the surface is where most of the phenomena dictating physical and chemical properties of the nanocrystal take place. 68,198 This also applies when it comes to doping. 

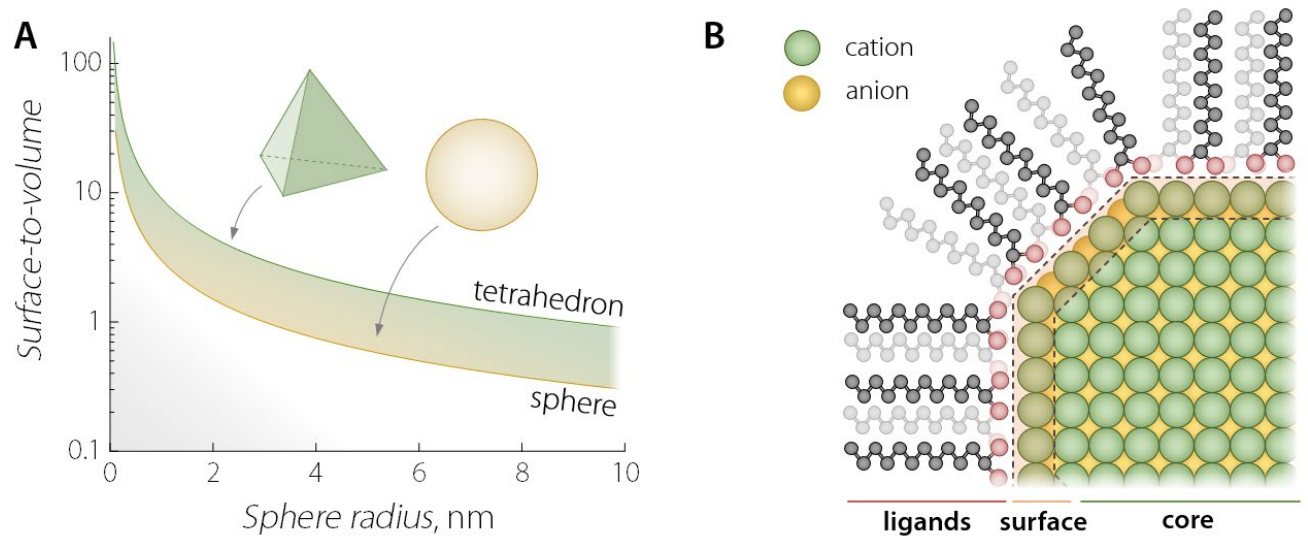

Figure 8. A: Surface-to-volume ratio vs size for two extreme cases of a sphere (yellow) and a tetrahedron (green). Most SNCs have a morphology that falls between these two shapes. In the case of a tetrahedron, the ratio was plotted against the radius of the sphere that circumscribes it. B: Sketch of a SNC, its subdivision into core, surface, and ligands tethered to the SNC surface.

Indeed, for an impurity/dopant ion to be incorporated in the lattice, it should first adsorb to the surface. ${ }^{199}$ From there, diffusion towards the core might occur, or further material can be added on top of the impurity thus trapping the dopant in the nanocrystal. Clearly, the residence time of the impurity at the surface is pivotal. ${ }^{191,198,200}$ But there are yet other aspects to which one should pay attention. As we discuss further below, attempts to dope a colloidal SNC can sometimes result in mere adsorption of the impurity ion at the surface. Even when the dopant is retained within the core, homogeneously doping an ensemble of SNCs is challenging; in fact, particle-to-particle variability in the doping density, which follows a Poisson distribution ${ }^{201}$, is generally unavoidable. Heavy doping of a SNC will induce lattice distortions, not to mention changes in the electronic band structure. ${ }^{198}$ In the case of $\mathrm{Ln}^{3+}$-doping for optical applications, this can be regarded as a secondary concern, since the concentration of these ions is usually kept rather low in order to avoid concentration quenching phenomena. ${ }^{202,203}$ Finally, the aliovalent doping of $\mathrm{Ln}^{3+}$ introduces defects (e.g., interstitial anions or vacancies thereof) in the nanocrystal for charge neutrality of the structure. To alleviate possible defect-induced loss of structural integrity, co-doping with a second element that compensate for charge mismatch could be a viable approach. ${ }^{204}$ With this in mind, let us briefly enumerate the synthesis methods developed for the preparation of doped colloidal SNCs.

\subsection{A game of precursor reactivity control}

The synthesis of doped colloidal SNCs has been perfected over the years, with more and more refined strategies being developed. These methods make use of metal precursors in the form of either single-source precursors (SSPs) or metal salts (Figure 9A). SSPs are metal complexes where the desired metal-anion bond is already present or whose decomposition locally generates highly reactive anionic species. Doped colloidal SNCs can be prepared thermally decomposing a mixture of homometallic SSPS in solution, which individually contain dopant and host metals. ${ }^{107,205,206}$ Alternatively, heterometallic SSPS can be used. ${ }^{207,208}$ SSPs can also be produced in-situ upon addition of the metal cation and the desired ligand directly in the reaction flask. When metal salts are employed, an anion source should be introduced in the reaction environment to induce nucleation and growth of the SNCs. ${ }^{209,210}$ 
A

Type of precursors*

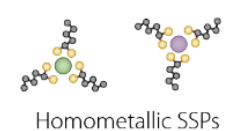

host
metal $0_{0}^{\circ}$

Metal ions

C

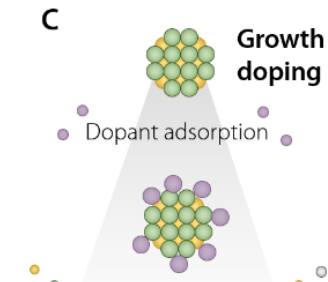

$\therefore$ Material overgrowth $\circ$

i

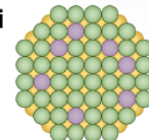

ii

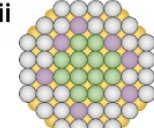

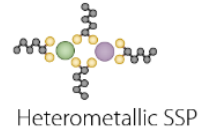

Heterometallic SSP

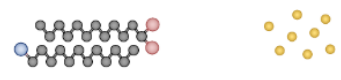

Anion source
B

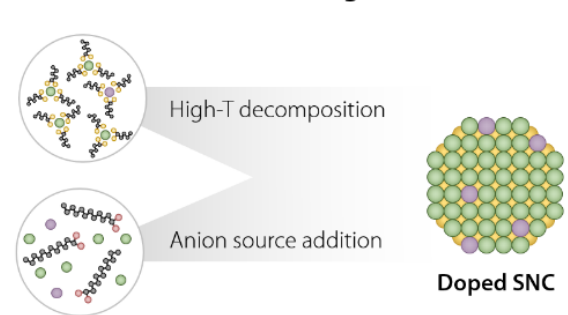

D

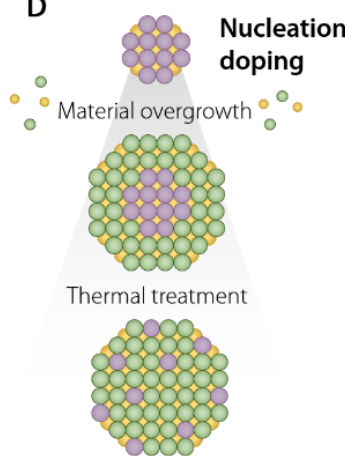

E
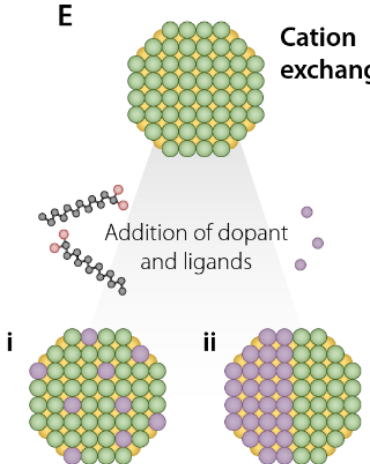

$\mathrm{F}$

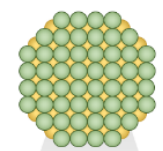

Diffusion doping

SSPs: dithiocarbamates, oleates, acetylacetonates, alkoxides.

Metal sources: chlorides, bromides, iodides, nitrates, acetylacetonantes, acetates, oxides, carbonates.

Ligands: oleylamine, octylamine, trioctylamine, oleic acid, trioctylphosphine, tributylphosphine, trioctylphosphine oxide, dodecanethiol, glycols, mercaptopropionic acid, polyvinyl pirrolidone, citrate..

Anion source: $\mathrm{S}, \mathrm{Se}$, Te, dodecanethiol, hexamethyldisilathiane (bis(trimethylsilyl)sulfide), $N, N^{\prime}$-diphenyl thiourea, $\mathrm{Na}_{2} \mathrm{~S}, \mathrm{NaOH}$, chlorotrimethylsilane...

Figure 9. Overview of methods for the preparation of doped SNCs. A: Precursors for the synthesis can be homoor heterometallic complexes (single-source precursors - SSPS) already containing the metal-anion bond; alternatively, metal salts are mixed with ligands and then an anion source is introduced in the reaction environment. Some of the most common species employed for the synthesis of doped and undoped SNCS are reported in the grey square at the bottom of the figure. B: Statistical mixing. C: Growth doping. The shell material can be the same of the "seed" SNC (i) or different (ii). D: Nucleation doping. E: Cation exchange. The exchange can take place isotropically (i) or preferentially at one crystal facet (ii). F: Diffusion doping.

The presence of suitable coordinating species (ligands) ensures control over the growth of the material. It is also well-established knowledge that these ligands impart colloidal stability to the final nanocrystals by remaining tethered to their surface. Both types of precursors, and combinations thereof, have been explored for the synthesis of colloidal LnSNCs and doped SNCs in general.

The simplest method for the synthesis of doped SNCs relies on statistical mixing of the dopant and host metal ions (Figure 9B). To obtain incorporation of the dopant in the semiconductor lattice with this approach, the reactivity of the precursors should be close (i.e., similar decomposition temperature) and/or the additional ligands introduced in the reaction environment should compensate for discrepancies. When the conditions described in Section 3.1 are satisfied (particularly regarding the CN of the cation to be substituted), it is indeed possible to rely on statistical mixing to obtain LnSNCs. For instance, $\mathrm{CaS}: \mathrm{Ce}^{3+}, \mathrm{Er}^{3+} / \mathrm{Nd}^{3+} \mathrm{SNCs}$ were recently prepared by Zheng, Chen and co-workers, ${ }^{109}$ and there are several instances of halide perovskites prepared in this way (see Section 6.3). Nonetheless, it is not straightforward to achieve such balance of the reactivity, even more so when $\mathrm{Ln}^{3+}$ doping (hard Lewis acids) is attempted in SNCs (which are often composed of metals with softer Lewis acids behavior Figure 10).

Often, doping requires more refined strategies, where a scrupulous control over the nature and reactivity of the species in the reaction mixture is known and leveraged. To that end, we can identify at 


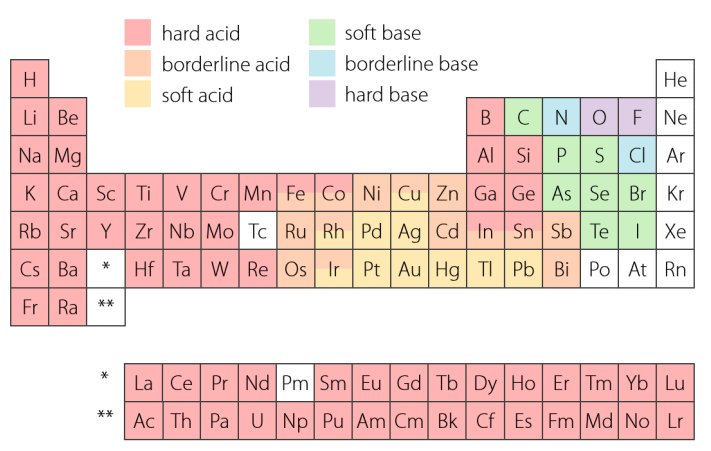

Figure 10. Strength of Lewis acids and bases. Similia similibus solvuntur (i.e., like dissolves like) ${ }^{211}$ or, in this case, "coordinatur", since ligands containing hard (soft) bases will coordinate more strongly hard (soft) acids. Some elements have different character depending on the oxidation state. The box of those elements is divided in two: the upper part is for the higher stable oxidation state, the bottom part for the lower oxidation state (e.g., $\mathrm{Fe}^{3+}$ upper, $\mathrm{Fe}^{2+}$ bottom). Adapted with permission from Ref. ${ }^{191}$. Copyright 2013 American Chemical Society.

least four main approaches: growth doping, nucleation doping, cation exchange, and diffusion doping. Growth doping (Figure 9C) entails the formation of a "seed" SNC, over which the dopant ions are adsorbed. This step is followed by the overgrowth of a layer of material (shell) that traps the dopants in the lattice. Using this approach, the dopant is mainly found in the SNC at a depth roughly corresponding to the radius of the initial seed. ${ }^{212}$ That is if the shell of additional material is grown at a temperature low enough to preclude excessive diffusion and ejection of the incorporated ions. ${ }^{107,213}$ To guarantee this, the precursor(s) used for the growth of the shell should be sufficiently reactive so to avoid exceedingly high temperature in the last shelling step. In nucleation doping, (Figure 9D) the seed nanocrystal contains only the dopant element. ${ }^{214,215}$ A shell of the targeted host material is then grown on top of it and homogeneous distribution of the dopant is achieved via "wet annealing" - which induces diffusion of the impurities towards the surface. ${ }^{216}$ Like in growth doping, the temperature at which this annealing is conducted is critical, since extended annealing or temperatures that are too high could result in ejection of the dopant ions. The temperature for the growth of the shell should be carefully controlled too, so to avoid occurrence of Ostwald ripening phenomena involving the "seed" SNCs. An example of how to select the proper strategy among growth and nucleation doping, and the subtle aspects underpinning this choice, can be found in a work by Meijerink's group (see Section 6.4). ${ }^{107}$ There, $\mathrm{Ce}^{3+}$ and $\mathrm{Eu}^{2+}$-doped AESs were prepared using single-source precursors.

Another powerful approach is cation exchange (Figure 9E), ${ }^{*}$ which proceeds much faster at the nanoscale compared to bulk, owing to its surface-driven nature. This method has been perfected over the years and it allows preparing a wealth of materials that are otherwise not accessible by other means. ${ }^{217-219}$ It entails the preparation of nanocrystals, followed by their dispersion in a solution containing the cationic species to be incorporated. Suitable ligands are also added, whose role is to promote the exchange and control the kinetics of the process. It is important to notice that during cation exchange, the anionic sublattice remains unaltered. In this context too, a fine control over the affinity of the species involved is key. If the ligand coordinates too effectively the dopant ion in solution, the exchange is hampered. At the same time, organic species with a stronger affinity towards the cation in the lattice promote its extraction and substitution. The group of Manna capitalized in several instances on this approach. ${ }^{70,218,220}$ In one study, they also demonstrated that the presence of cation vacancies act as a promoter for the exchange of $\mathrm{Cu}^{+}$for $\mathrm{Cd}^{2+}$ in copper-deficient $\mathrm{Cu}_{2-\mathrm{S}} \mathrm{S}$ nanocrystals. ${ }^{220} \mathrm{Yb}^{3+}$-doped

\footnotetext{
*Anion exchange strategies exist, and are routinely used to prepare, e.g., $\mathrm{CsPb}\left(\mathrm{Cl}_{x} \mathrm{Br}_{1-x}\right)_{3}$. However, the lower mobility of larger anions has limited the development of anion exchange approaches.
} 
$\mathrm{CsPb}_{3}, \mathrm{CsPbBr}_{3}$, and $\mathrm{CsPbCl}_{3}$ were also obtained using this method. ${ }^{221}$ An example of how to control the properties of LnSNCs via cation exchange is presented in the last section of this Review (Section 6.2).

A close relative of cation exchange is doping via diffusion (Figure 9F), an approach that relies on the simultaneous addition of cations and anions in the structure. ${ }^{193}$ This method allows retaining the crystal shape, structure, and size distribution of the original nanocrystals. But it has the limit of not being readily applicable to cations that readily react with the anionic species introduced in the solution - which are also present in the host lattice. We can regard diffusion as a "thermodynamic variant" of nucleation doping (Figure 9C): instead of trapping the impurities in the nanocrystal upon addition of further material on the surface, the adsorbed ions are driven inside the lattice by entropic factors. ${ }^{193}$ Although to date no $\mathrm{Ln}^{3+}$ doping of SNCs has been attempted via this method, it is foreseeable that the low reactivity of $\mathrm{Ln}^{3+}$ towards heavy chalcogens could be leveraged to prepare new LnSNCs.

Given the surface-driven nature of several of the discussed approaches, one often finds that the processes are non-isotropic. This is because adsorption of the species in solution is favored on some facets of the nanocrystals over others. ${ }^{222,223}$ It is also interesting to note that depending on the miscibility of the chosen materials one can obtain segregation of phases, with the creation of highly asymmetric structures (e.g., Janus nanoparticles). ${ }^{218}$

Overall, the methods above offer several avenues for the preparation of a plethora of nanomaterials with the most diverse optical and electronic properties, as well as surface chemistry. Some have proven more suitable than others in controlling the doping density and the radial distribution of the dopants. Combination of thermodynamic and kinetic control allows achieving and stabilizing at the nanoscale phases previously inaccessible in bulk form. However, a priori selection of the proper dopant-host combination according to the guidelines provided in the previous sections remains imperative, since it allows increases the chances of successful and efficient incorporation of $\mathrm{Ln}^{3+}$ in the nanocrystal.

4.3. Surface engineering. This section would not be complete with a mention to methods to alleviate the issue of surface quenching. In that vein, we have already discussed in Section 4.1 that the surface of nanoparticles exhibits a plethora of sites responsible for the quenching of the luminescence. In SNCs, ligand- and defect-related states can act as charge traps (Figure 11). ${ }^{68,} 224$ Moreover, the vibrations of ligands or other molecules interacting with the surface (together with the intrinsic lattice vibrations - phonons) can strongly support non-radiative de-excitation events. ${ }^{68}$ FRET to molecules attached to the surface could also effectively quench the emission of the SNC. ${ }^{225}$ These phenomena (along with Auger recombination ${ }^{226}$ ) are responsible for losses in the emission intensity of SNCs. In the specific case of LnSNCs, trapping of charge carriers and non-radiative recombination events at the surface ultimately yield to a reduced probability of population of the lanthanide's emitting state. Surface treatments and growth of core/shell architectures have been developed to alleviate this issue in SNCs, and they are envisaged to prove effective also to increase the emission of LnSNCs. For example, in halide perovskites, the surface can be subjected to specific treatments to both reduce quenching and increase the SNC colloidal stability. ${ }^{227}$ Replacement of labile carboxylate ligands for phosphonates or zwitterionic molecules results in more effective and stable saturation of the coordination sphere of surface ions. ${ }^{228}$ Likewise, treatments with solutions of suitable metal halide salts (e.g., bromides, ${ }^{229,}{ }^{230} \mathrm{chlorides}^{231}$ and iodides ${ }^{232}$ ) allow saturating the anionic coordination sphere and promote better binding of the ligands. In a similar fashion, $\mathrm{Yb}^{3+}$ ions found at the surface of CdSe SNCs were shown to emit more intensely when the system was subjected to a treatment with a Se-TOP solution in ODE, due to better saturation of the coordinating sphere of the ions. ${ }^{49}$ The fundamental role of an effective passivation of SNC surface in granting bright emission was observed also in more classical SNCs, such as PbSe and CdSe. ${ }^{233} \mathrm{Hassinen}$ et al. showed how protic polar solvents (i.e., alcohols) employed during the purification step of SNCs can strip carboxylate ligands due to reprotonation of the acidic moiety. This, in turn, results in a more defective surface, ultimately yielding poor emission efficiency. 

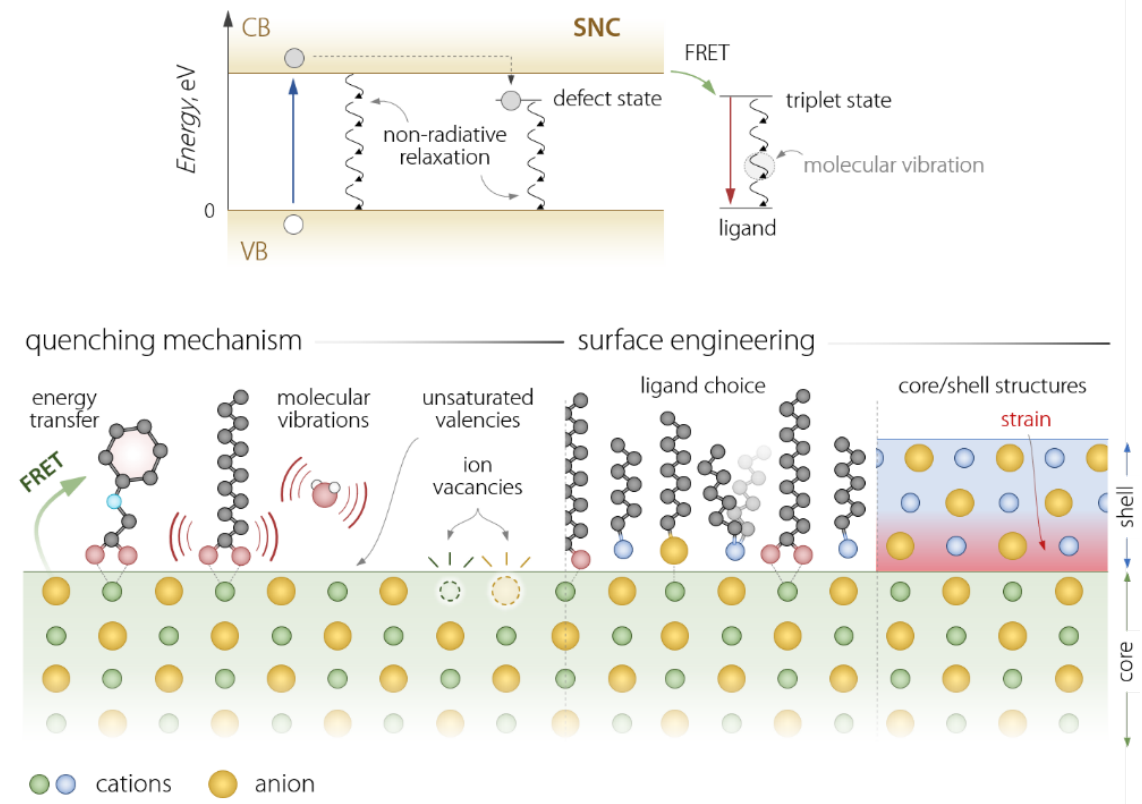

Figure 11. What happens at the surface of a SNC greatly impacts the optical performance of the system. After an electron-hole pair is generated within the SNC via photoexcitation (blue vertical arrow), vibrations of external molecules (attached ligands or solvent molecules) can promote non-radiative relaxation processes. Charge carriers can also get trapped at interbandgap defect-related states that generates from unsaturated bonds, ion vacancies and distortions at the surface. The ligands tethered to the surface might also act as energy acceptors in FRET-like energy transfer mechanisms. The energy received can be released as emitted light (red vertical arrow) or nonradiatively through molecular vibrations. Strategies to reduce the impact of surface states entail the choice of suitable ligands that firmly attach to the SNC and the overgrowth of a shell that effectively "moves the surface away" from the core SNC and saturate the valency of the core ions. When opting for this latter strategy, strain at the core/shell interface is generated (red halo), due to lattice mismatch between the materials.

Therefore, arguably the most exploited strategy for suppressing surface effects is the preparation of core/shell architectures (Figure 11) ${ }^{234}$ This shell of material grown atop the SNC core simultaneously saturates the coordination sphere of the ions at the interface between core and shell, and creates a physical barrier between the optically active core and external molecules responsible for luminescence quenching (ligands and/or solvent molecules). The overall result is a SNC with stronger emission intensity. There are various types of core/shell architectures for SNCs, depending on the relative position of the VB and CB of the core and shell material. ${ }^{234}$ Generally, when brightly emitting SNCs are sought after, type-I architectures are preferred. In this configuration, the bandgap of the shell material is larger than the one of the core, and the VB (CB) of the shell is found below (above) the VB (CB) of the core. An important parameter to consider for a successful growth of a shell is the lattice mismatch between the core and shell material. Large lattice mismatch and different crystals structures are bound to hinder the growth of a shell or to generate a substantial stress at the core/shell interface. 235,236 To relive this stress, dislocations and defects are created, which can act as luminescence quenchers. This latter aspect is true also for materials with relatively small lattice mismatch, like in the iconic CdSe/ZnS quantum dots (12\% mismatch ${ }^{235}$ and even in PbSe/PbS, where the lattices almost perfectly match (3\% mismatch). ${ }^{237}$ Moreover, depending on the synthesis conditions, cation exchange processes might occur in place of or in parallel with epitaxial growth of the shell. ${ }^{238}$ It is worth highlighting that shelling has proven challenging for halide perovskite SNCs, owing to the strong ionic nature of the lattice. ${ }^{239}$ However, strategies have recently been proposed that result in core/shell perovskite $\mathrm{SNCs}{ }_{1}{ }^{24-242}$ also using $\mathrm{SiO}_{2}{ }^{243}$ and $\mathrm{TiO}_{2}{ }^{244}$ as coating materials. These studies open the door to the development of even more efficient and stable LnSNCs based on halide perovskites. 


\section{Characterization techniques}

Proper characterization of nanomaterials is a challenging matter per se. A combination of different techniques is required to fully understand the structure at the nanoscale and its relationship with physicochemical properties. Theoretical modelling of the properties in a "blind" way (when experimental results are not known to theoreticians) can also be extremely beneficial. We will not dwell on an in-depth discussion about how a generic nanoparticle should be characterized, since a very instructive Review was recently published on the subject by Modena and Wuttke. ${ }^{245}$ We will instead suggest the more appropriate characterization methods, and combination thereof, to assess whether doping has been successful and how to evaluate the impact of doping on the optical properties of the nanocrystals.

\subsection{Structural and compositional characterization}

The small size of a SNC translates to a limited number of atoms per particle (tens to tens of thousands). If we aim for a $\mathrm{Ln}^{3+}$ doping of few percentage points, statistically we might have as little as one single impurity ion per nanocrystal. How do we assess the presence of the dopant ion in the particle in this challenging situation? Is the lanthanide adsorbed on the surface or rather incorporated in the nanocrystal? Can we leverage the optical properties of lanthanide ions to obtain information of their location?

To establish the presence of dopant in the sample, elemental analysis is the prime tool either in the form of mass spectroscopy (MS) or atomic emission spectroscopy (AES). Along the same line, total X-ray fluorescence (TXRF) spectroscopy grants accurate quantification of the elements in the system. ${ }^{246}$ Nonetheless, neither of those methods provide information as to where the dopant ion is localized (adsorbed at the surface or retained at some depth throughout the nanocrystal lattice). Some indirect evidence can be gathered using other approaches, and those based on the interaction of X-rays with the sample are particularly useful. X-ray absorption spectroscopy (XAS - either as extended X-ray absorption fine structure (EXAFS) or X-ray absorption near-edge spectroscopy (XANES)) is an insightful technique since it allows obtaining precise information about the oxidation state and chemical surrounding of the observed elements. Variations of the average coordination sphere(s) (coordination number - $\mathrm{CN}$ ) of the element of interest, changes in the bond length between elements, and the site occupied by the dopant can be evaluated. For example, in $\mathrm{BaTiO}_{3}: \mathrm{Eu}^{3+}$ nanocrystals, XANES and EXAFS analysis allowed identifying oxidation state, $\mathrm{CN}$, and Eu-O bond length as a function of dopant content

(Figure $12 \mathrm{~A}, \mathrm{~B}, \mathrm{C}){ }^{247}$ In that situation, the fact that in the XANES spectra the position of the absorption edge of europium is the same for $\mathrm{BaTiO}_{3}: \mathrm{Eu}^{3+}\left(x^{\circ} \%\right)$ and $\mathrm{Eu}_{2} \mathrm{O}_{3}$ signifies that $\mathrm{Eu}^{3+}$ is in its trivalent oxidation state in the nanocrystals. Given the paramagnetic nature of most rare-earths, electron paramagnetic spectroscopy (EPR) can also be used to investigate the coordination environment of the dopant and its magnetism. ${ }^{248}$ An example of the use of this technique in LnSNCs can be found in the analysis of the EPR spectra of Eu-doped CaS (Figure 12D). ${ }^{164}$ The authors observed two sets of six-line patterns arising from the two ${ }^{151} \mathrm{Eu}^{2+}$ and ${ }^{153} \mathrm{Eu}^{2+}$ isotopes. Analysis of the hyperfine coupling constants for the LnSNCs and comparison with the values of bulk material confirmed the location of europium at $\mathrm{Ca}^{2+}$ cubic site. Powder X-ray diffraction (PXRD) patterns of doped and undoped samples can be compared to retrieve information regarding changes in the lattice parameters. ${ }^{51,249}$ Differences in the ionic radius of the dopant and host cation result in contraction or expansion of the average unit cell, that in turn affects the position of the diffraction reflections. Hence, a qualitative indication of the successful incorporation of the dopant can be drawn from the analysis of the PXRD patterns. Nonetheless, the appearance of additional defects in the structure might balance out this effect and sometimes, even at high doping concentrations, negligible shifts are observed. ${ }^{56,84}$ X-ray photoelectron spectroscopy (XPS) is a technique that allows probing the first few nanometers of a material. In the case of small nanoparticles, this length 

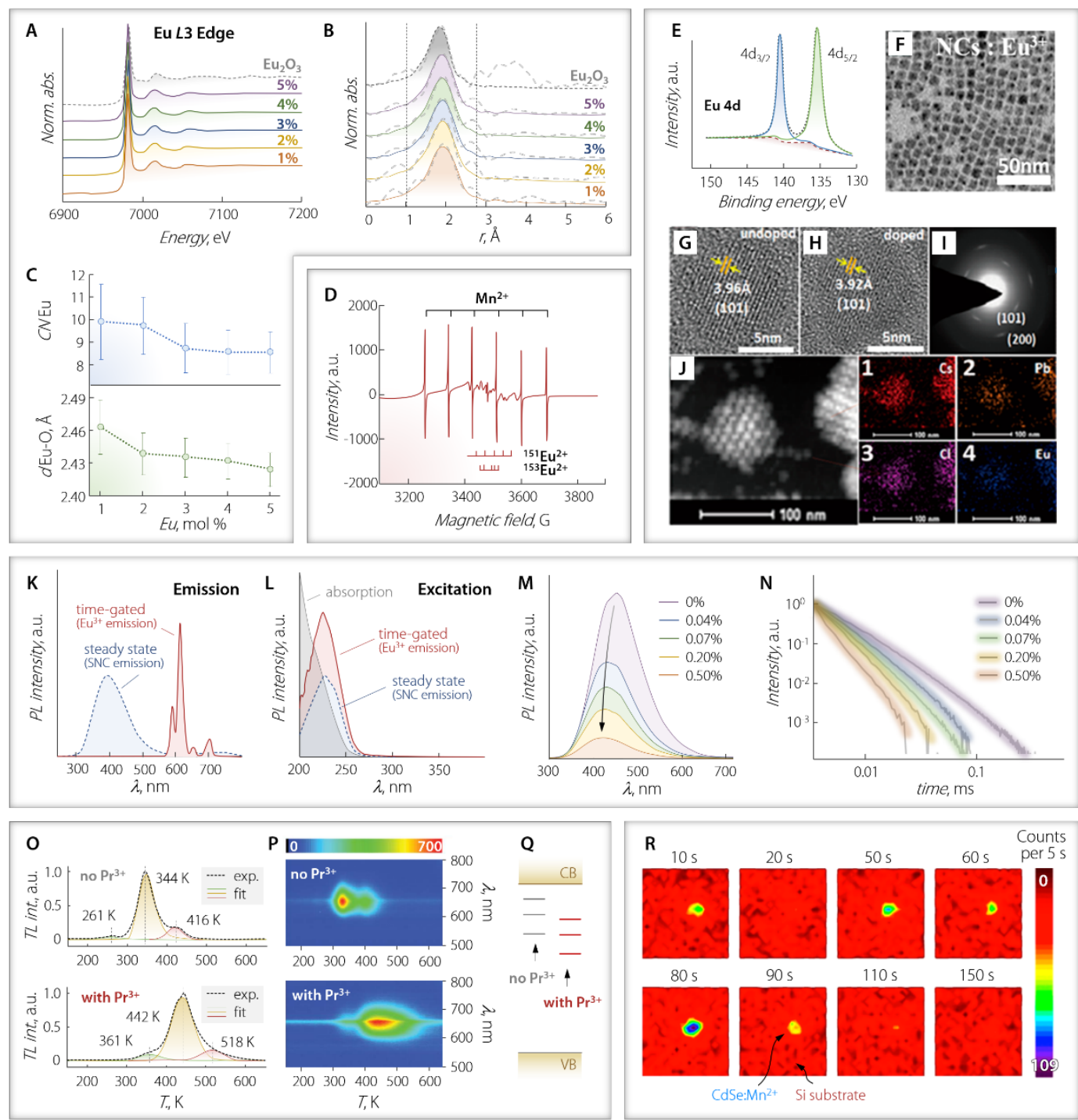

Figure 12. Practical examples of characterization techniques employed for the study of LnSNCs. A-C: XAS characterization of $\mathrm{BaTiO}_{3}: \mathrm{Eu}^{3+}(x \%)$ nanocrystals. XANES spectra (A) along with the Fourier transform of the experimental EXAFS spectra (solid lines) overlaid with first-shell, single-scattering fits to the data (dashed lines) (B). Average Eu-O distance ( $d \mathrm{Eu}-\mathrm{O})$ and $\mathrm{Eu}^{3+}$ coordination number $\left(\mathrm{CN}_{\mathrm{Eu}}\right)$ as a function of nominal $\mathrm{Eu}^{3+}$ concentration (C). Adapted with permission from Ref. ${ }^{247}$. Copyright 2013 Royal Society of Chemistry. D: EPR spectrum of CaS:Eu ${ }^{2+}$ LnSNCs recorded at 300 K. Adapted with permission from Ref. ${ }^{164}$. Copyright 2002 Royal Society of Chemistry. E-J: characterization of $\mathrm{CsPbCl}_{3}: \mathrm{Eu}^{3+} \mathrm{LnSNCS}$. XPS spectrum of (Eu 4d edge - E). TEM image of Eu ${ }^{3+}$-doped LnSNCs (F) and HR-TEM images of undoped and doped $\mathrm{CsPbCl}_{3} \operatorname{SNCs}(\mathbf{G}, \mathbf{H})$. Electron diffraction pattern obtained during TEM

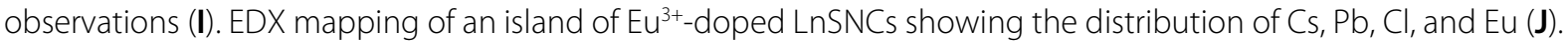
Adapted with permission from Ref. ${ }^{51}$. Copyright 2017 American Chemical Society. K-N: Optical characterization of $\mathrm{Eu}^{3+}$-doped $\mathrm{Ga}_{2} \mathrm{O}_{3}$ LnSNCs. Steady-state and time-gated emission spectra under host excitation $(230 \mathrm{~nm})$ show broad host emission (DAP-type) and $\mathrm{Ln}^{3+}$ sharp luminescence (K). Excitation spectra obtained monitoring the SNC emission ( $400 \mathrm{~nm}$ in steady-state mode) and Eu $\mathbf{u}^{3+}$ emission ( $615 \mathrm{~nm}$ in time-gated mode - $\mathbf{L}$ ). Quenching of DAP emission from the host upon increasing $\mathrm{Eu}^{3+}$ content $(\mathbf{M})$, mirrored by the shortening of the lifetime of DAP emission (N). Adapted with permission from Ref. ${ }^{93}$. Copyright 2014 Royal Society of Chemistry. O-Q: TL analysis of LnSNCs of CaS:Eu' (top) and CaS:Eur ${ }^{2+}, \mathrm{Pr}^{3+}$ (bottom). The TL curves can be deconvoluted using three Gaussian curves (0) whose center shifts towards longer wavelengths upon doping with $\mathrm{Pr}^{3+}$. In both LnSNCs, the emission is centered around $650 \mathrm{~nm}(\mathbf{P})$. The deconvolution in $\mathrm{O}$ is used to determine the energy of interbandgap trap states (Q). Adapted from Ref. ${ }^{126}$. R: single-particle photoluminescence analysis of a CdSe:Mn ${ }^{2+} \mathrm{LnSNC}$ attached to a Si substrate recorded at $5 \mathrm{~K}$, showing the blinking behavior of the particle. Adapted with permission from Ref. ${ }^{78}$. Copyright 2016 American Chemical Society. 
represents almost the total volume of the system. Using this method, qualitative information about the presence of a specific element can be retrieved, along with its oxidation state, type of bond, and relative abundance compared to other elements (Figure 12E). ${ }^{51,52,112,164}$

Seeing is believing, therefore the most reliable and direct method to assess the success in terms of doping is high-resolution transmission electron microscopy (HR-TEM - Figure 12F, G, H). Z-contrast i.e., contrast based on difference in the atomic number of the elements - might be useful in situations where the mass of the elements differs substantially, ${ }_{1}^{48}$ but discrimination of few $\mathrm{Ln}^{3+}$ ions per particle is hardly achieved without additional support. Most transmission electron microscopes are also equipped with energy-dispersive X-ray spectroscopy (EDX) detectors, which can provide elemental maps (Figure 12J). However, the best results are achieved when a high-resolution technique like high-angle annular dark filed scanning transmission electron microscopy (HAADF-STEM) is coupled with electron energyloss spectroscopy (EELS). ${ }^{250}$ This spectroscopy provides further information about the composition of the material and oxidation state of the elements, allowing observations down to a single ion per particle ${ }^{191}$ since EELS signals of different $\mathrm{Ln}^{3+}$ ions are discriminated with relative ease. ${ }^{251}$ Electron diffraction can also be employed and information similar to those obtained from PXRD can be extracted (Figure 12I). The difference there resides in the fact that unlike PXRD, which affords an information averaged over the nanocrystal ensemble, electron diffraction allows to gain knowledge about single nanocrystals. A better visualization of LnSNCs using TEM can be achieved subjecting the particles to a ligand exchange procedure, wherein organic ligands are replaced by iodide ions (using tetrabutylammonium iodide as the exchanging molecule). ${ }^{198}$ In this way, the interference of the carbonaceous organic molecules is minimized and a clearer observation of the nanocrystals becomes possible.

\subsection{Light interaction and generation}

Indirect evidence of the incorporation of dopants can be obtained from optical analysis. This is true for the incorporation of any dopant ion, since changes in the band structure are bound to occur possibly along with the creation of interbandgap states. $188,252,253$ Thus, comparison of absorption, emission, and excitation spectra, both in steady-state and time-resolved modality, along with transient absorption spectroscopy, represents a powerful tool to indirectly assess the presence and the extent of dopant incorporation. Here, we are interested in the specific information that can be derived in the case of $\mathrm{Ln}^{3+}$ doping. Seeking host-sensitization of $\mathrm{Ln}^{3+}$ emission, one should first check the appearance of emission lines typical of the introduced $\mathrm{Ln}^{3+}$ when exciting at wavelengths that promote electron excitation to the CB (Figure 12K). Photoluminescence excitation spectroscopy allows to gather direct evidence of this transfer (Figure 12L) by monitoring the emission of the doped lanthanide and scanning a range of excitation wavelengths..$^{93}$ If the so-obtained excitation spectrum resembles the excitation spectrum obtained for the undoped SNC, then host sensitization is occurring. In some situations, $\mathrm{Ln}^{3+}$ emission can be so dim that it is masked by the stronger emission of the host semiconductor. The long-lived nature of $4 \mathrm{f}-4 \mathrm{f} \mathrm{Ln}^{3+}$ photoluminescence $\left(10^{-6}-10^{-3} \mathrm{~s}\right)$, as opposed to the one of SNCs $\left(10^{-9}-10^{-7} \mathrm{~s}\right)$, allows to exploit time-gated measurement techniques to observe exclusively one or the other emission (Figure 12K, L). Emission of $\mathrm{Tb}^{3+}$ and $\mathrm{Eu}^{3+}$ in binary sulfides and selenides has been investigated in this way. ${ }^{53,54,98,163}$ However, when efficient sensitization of $\mathrm{Ln}^{3+}$ emission takes place, steady-state measurements suffice (see Section 6). ${ }^{50,51,54}$ In a way, granted a favorable situation in term of energy levels/bands position, the need for time-gated approaches is a discriminant for poor $\mathrm{Ln}^{3+}$ incorporation or suggests that the ions might reside on the SNC surface. In fact, if the dopant is incorporated in the lattice, it is better shielded from the external environment, and hence de-activation processes typical of surface states are reduced (see Section 4.3, as well as and further down Section 6.1 and 6.4).

The occurrence of host sensitization can be directly corroborated by photoluminescence excitation spectroscopy upon monitoring $\mathrm{Ln}^{3+}$ emission. The predominance of the contribution owing to host absorption versus the sharp $\mathrm{Ln}^{3+}$ lines is also a good indication of effective transfer taking place (see 
Sections 6.3 and 6.4) as well as the quenching of the emission of the host material (Figure $\mathbf{1 2} \mathbf{M}$ ) ${ }^{93} \mathrm{Ln}^{3+}$ lifetime measurements provide information regarding emission efficiency of the dopant ion upon comparison with the natural lifetime of the $\mathrm{Ln}^{3+}$ transition monitored. ${ }^{57,}{ }^{254}$ Complementary, quantification of lifetime shortening (if any) of the SNC intrinsic luminescence allows assessing the efficiency of the non-radiative energy transfer processes (Figure $\mathbf{1 2 N}$ ) ${ }^{93}$ The profile of the $\mathrm{Ln}^{3+}$ decay curve contains also some information about the location of the $\mathrm{Ln}^{3+}$ in the lattice, since in principle it is possible to correlate the number of components required to fit the decay curve to the number of sites occupied by the emitter. Components with shorter decay times are associated with surface ions (them being more subjected to quenching phenomena), while longer-lived components are generally indicative of an emitting ion well incorporated in the lattice. ${ }^{49,98}$ It is not uncommon however to encounter a non-exponential decay profile. ${ }^{255}$ This behavior stems from the presence of defects and different coordination environments that a $\mathrm{Ln}^{3+}$ dopant can experience in a SNC (Figure 8B) as well as SNC-to-SNC variability, which translate to several populations of emitting centers with different chemical surrounding. By the same token, when $\mathrm{Ln}^{3+}$ ions occupy surface states and/or several sites in the crystal lattice, the photoluminescence spectrum is characterized by rather broad and featureless bands. ${ }^{49}$ However, when sharper lines are observed, and the fine structure of the transitions can be resolved, siteselective spectroscopy can be performed. A thorough Review has recently addressed this subject in LnSNCs. ${ }^{256}$ Yet, even when such refined analysis is not possible because of insufficiently resolved emission lines, qualitative information regarding the symmetry of the occupied site can be obtained for some $\mathrm{Ln}^{3+}$ ions considering the ratio between the intensity of so-called electric (ED) and magnetic dipole (MD) transitions. MD transitions are poorly influenced by the coordination environment, while the intensity of ED transitions (also called hypersensitive) decreases in high-symmetry crystallographic site. The classical case is the one of $\mathrm{Eu}^{3+},{ }^{257}$ with its ${ }^{5} \mathrm{D}_{0} \rightarrow{ }^{7} \mathrm{~F}_{1}(\mathrm{MD})$ and ${ }^{5} \mathrm{D}_{0} \rightarrow{ }^{7} \mathrm{~F}_{2}$ (ED) transitions, but several other $\mathrm{Ln}^{3+}$ ions feature similar bands. ${ }^{147,258}$ This ratio (ED/MD) is often referred to as the $R$ value and the larger it is the more asymmetric is the site occupied by the emitting ion. ${ }^{259}$ Last, but not least, the PLQY of LnSNCs can be determined in relative or absolute terms. ${ }^{260}$ The relative PLQY is obtained by comparison with a species featuring known emission efficiency (e.g., Rhodamine B, Indocyanine Green). Absolute PLQY measurements are more challenging and require the use of an integrating sphere, which allows collecting $100 \%$ of the light emitted under photoexcitation. Reporting these carefully determined values is of utmost importance to ensure comparison of the performance of newly developed materials.

As we have previously discussed (see Section 3.2.1) the introduction of a dopant, such as a $\mathrm{Ln}^{3+}$ ion, might introduce or stabilize localized defect states. These states can trap charge carriers (e.g., electrons) within the bandgap, thus enabling phenomena such as afterglow and NIR-triggered visible luminescence. ${ }^{106,126}$ To investigate the position of these energy states, thermoluminescence (TL) is the technique of choice (Figure 120, P). A TL measurement entails increasing the temperature of the sample to induce thermal de-trapping of charge carriers occupying trap states within the bandgap. One or more emission peaks are generally observed, and the temperature at which each of them occurs is converted to energy (Figure 12Q) ${ }^{261}$ In the case of electron trap states, this value is the energy difference between the trap state and the bottom of the CB. Care must be exercised with this technique, since some materials can undergo phase transformation upon heating at high temperatures.

Lastly, a more sophisticated technique is constituted by single-particle spectroscopy (SPS - Figure 12R), which can proceed, for instance, via immobilization of the nanoparticles on a substrate or trapping using optical tweezers. ${ }^{262}$ This method allows to study the stability and relative brightness of single particle within a batch constituted by an ensemble of particles, thus providing information regarding the homogeneity of the sample. Although we could not find use of this technique for colloidal LnSNCs, it has been used in several instances to study the behavior of single $\left(\mathrm{Mn}^{2+}\right.$-doped) quantum $\operatorname{dots}^{78}$ and lanthanide-doped fluoride nanoparticles. ${ }^{263}$ 
In the previous sections we have provided a general overview of the aspects that one should consider when designing a colloidal LnSNC from the material standpoint and its synthesis, as well as of the means to confirm the success in the production of said nanomaterial. Before moving to address specific cases more in detail in the next section, we provide in Figure $\mathbf{1 3}$ a summary of the most important aspects discussed so far.

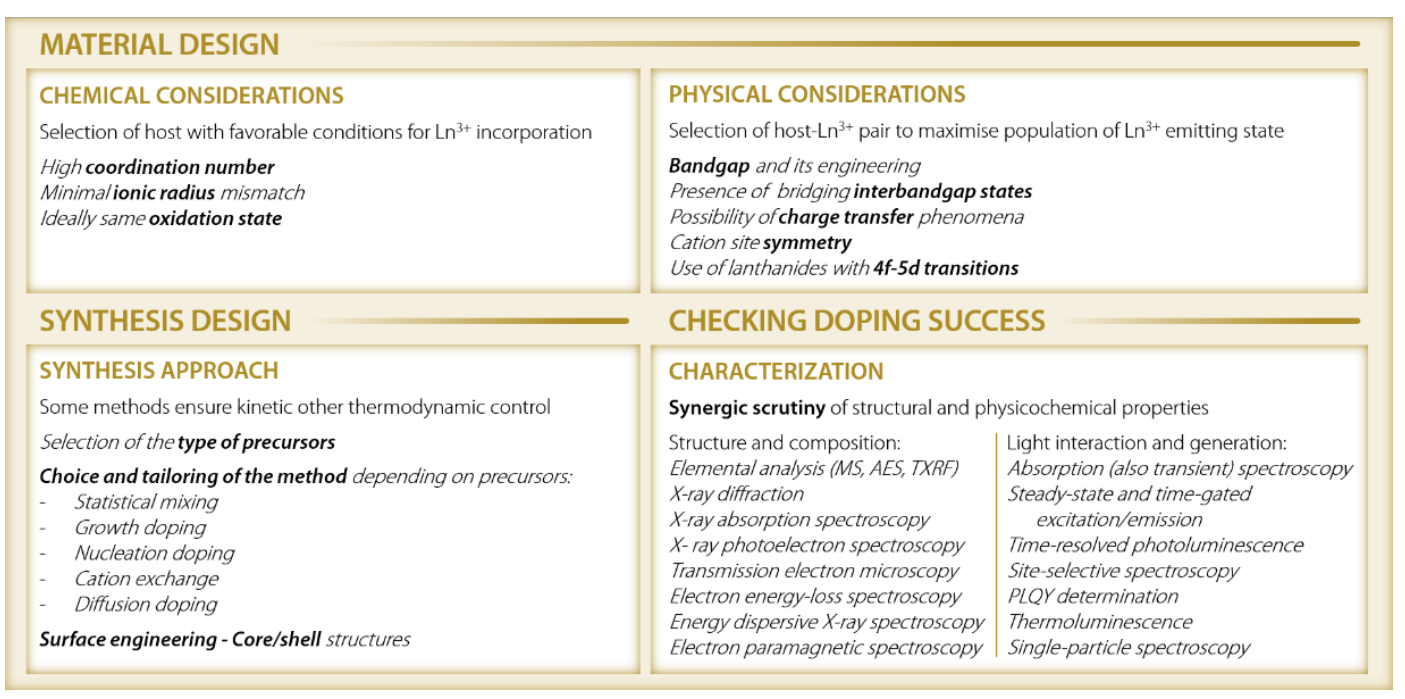

Figure 13. Summary of the parameters to be considered in the development of a colloidal LnSNC in terms of material and synthesis approach design, along with the most relevant characterization techniques for a complete investigation of the material's properties.

\section{Case studies}

In this section, we have selected four classes of host materials studied in the past few years for the preparation of LnSNCs. In particular, the host materials herein discussed are $\mathrm{CdSe}$, $\mathrm{Pbln}_{2} \mathrm{~S}_{4}$, halide perovskites, and AESs. Each subsection focuses on one or a limited number of investigations that we consider to be the most relevant and/or complete. By selecting few studies, we can get into the fine details of each of them, exploring the main aspects we have underlined in previous sections of this Review in terms of material design, synthesis method, and characterization of the LnSNC.

\subsection{Case of study I: CdSe:Yb ${ }^{3+}$}

$\mathrm{CdSe}$, in its wurtzite polymorph (the one stable at room conditions - space group $P 6_{3} m c$ ), features $\mathrm{Cd}^{2+}$ in a tetrahedral coordination environment. This is already a limiting factor for the substitutional incorporation of $\mathrm{Ln}^{3+}$ in the lattice, which is further hindered by mismatch in the oxidation state of the dopant and host cations. To overcome this hurdle, the group of Meijerink adapted a growth doping strategy initially devised for the preparation of $\mathrm{Mn}^{2+}$-doped CdS/ZnS nanocrystals. ${ }^{264}$ They used CdSe seeds, on top of which $\mathrm{Yb}^{3+}$-acetate was allowed to adsorb. Yb ${ }^{3+}$ ions were subsequently "trapped" upon injection of a Se solution that formed a shell on the SNCs (Figure 14A). From a physical standpoint, the sensitization of $\mathrm{Yb}^{3+}$ by $\mathrm{CdSe}$ was expected to take place, since the bulk bandgap is $1.74 \mathrm{eV}$ and the emitting state of $\mathrm{Yb}^{3+}$ is located at approximately $1.25 \mathrm{eV}$. Moreover, CdSe has a Bohr radius of $5.8 \mathrm{~nm}$, and the nanocrystals herein produced have a size of $\sim 4 \mathrm{~nm}$. Thus, the quantum confinement effect pushes the bandgap at even higher energy - approximately $2 \mathrm{eV}$, as evinced from the absorption spectra reported in the study. Indeed, host-sensitized emission from $\mathrm{Yb}^{3+}$ was observed (Figure 14B). The presence of $\mathrm{Yb}^{3+}$ ions in the sample was confirmed via EDX spectroscopy during TEM observation (Figure 14C), but their position in the nanocrystals during the different steps of the synthesis process 
A

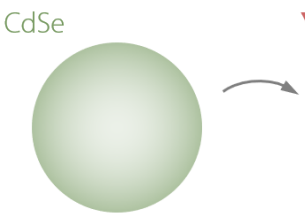

C

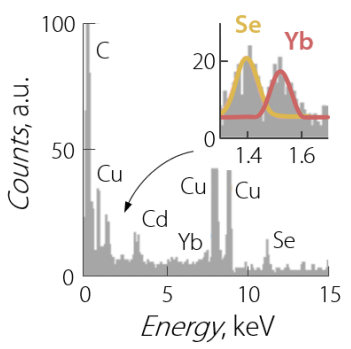

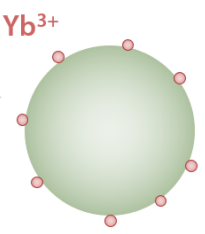

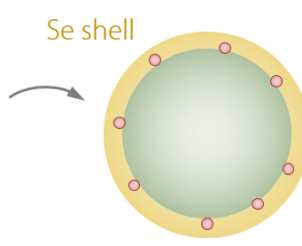

D

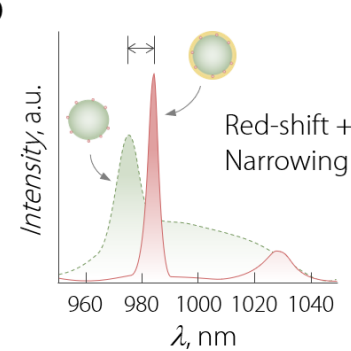

B

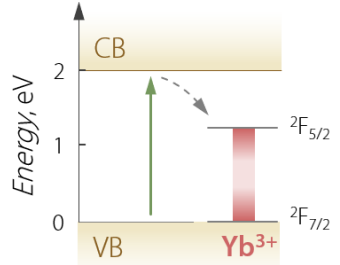

$\mathrm{E}$

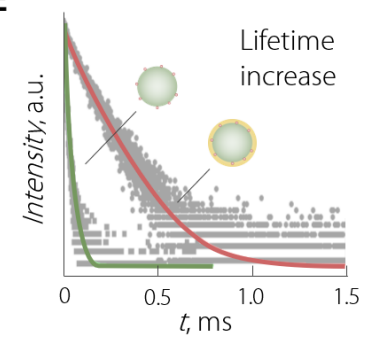

Figure 14. Trapping $\mathrm{Ln}^{3+}$ at the surface. A: graphical representation of the growth doping approach for the synthesis of CdSe:Yb ${ }^{3+}$. B: Energy level scheme as derived from the information in Ref. ${ }^{49}$. C: EDX spectrum acquired during TEM observation. Inset: Zoom-in of the 1.3-1.7 keV range, where the signals from Se and Yb are resolved. $\mathbf{D}$ : Comparison of the emission spectrum of CdSe:Yb ${ }^{3+}$ before (green) and after (red) Se shell growth. The spectra were recorded upon $580 \mathrm{~nm}$ excitation. The spectra are smoothened from the data reported in the manuscript. E: Photoluminescence decay curves recorded before and after Se shell growth upon exciting at $580 \mathrm{~nm}$ and monitoring $\mathrm{Yb}^{3+}$ emission. Adapted with permission from Ref. ${ }^{49}$. Copyright 2013 American Chemical Society.

was monitored indirectly by spectroscopic means (Figure 14D and E). $\mathrm{Yb}^{3+}$ adsorption on the nanocrystal surface (before Se shell growth) was confirmed upon observing a marked decrease of the emission intensity of the lanthanide after the system was subjected to a washing cycle - which detached a good portion of adsorbed ions. Moreover, the relatively broad $\mathrm{Yb}^{3+}$ emission was located at approximately $975 \mathrm{~nm}$. Upon growing the Se shell, $\mathrm{Yb}^{3+}$ emission became sharper and shifted to longer wavelength $\left(985 \mathrm{~nm}\right.$ ). This points towards a different environment experienced by Yb ${ }^{3+}$ (nephelaxeutic effect, particularly strong in the presence of polarizable anions and for early or late lanthanides) and a better shielding form the outer environment. Further confirmation of successful $\mathrm{Yb}^{3+}$ inclusion in the SNC after Se shell growth came from lifetime measurements: the decay time increased from 27 to 160 $\mu \mathrm{s}$, because of the reduced interaction with solvent and capping molecules found at the colloidal SNC surface.

\subsection{Case study II. Pbln $\mathrm{S}_{4}: \mathrm{Yb}^{3+}$}

Recently, the group of Gamelin reported on the preparation of a new type of LnSNCs combining statistical mixing approach and cation exchange. NalnS 2 was selected as the sacrificial material. The authors prepared $\mathrm{Yb}^{3+}$-doped nanocrystals of this ternary sulfide by mixing the metal precursors in the form of In- and Yb-acetylacetonate and Na-oleate along with sulfur powder as the anion source. The incorporation of $\mathrm{Yb}^{3+}$ in the semiconductor lattice was afforded by the presence of octahedral sites occupied by $\mathrm{In}^{3+}$. Moreover, $\mathrm{In}^{3+}$ is chemically similar to $\mathrm{Yb}^{3+}$, with comparable ionic radius, same oxidation state, and a hard Lewis acid behavior - which ensures a similar reactivity of the adducts formed between the two metal ions and the ligands in solution. This is a clear example of how an a priori selection of the host material allows minimizing the challenges during the synthesis stage, yet it does not completely eliminate them. Indeed, the synthesis parameters were finely controlled to maximize the incorporation of the lanthanide in the SNCs, selecting temperatures higher than $280^{\circ} \mathrm{C}$ and S:Yb ratio above 10. Even then, only $10 \%$ of the $\mathrm{Yb}^{3+}$ introduced in the reaction environment was incorporated in the LnSNCs. 
Host-sensitized $\mathrm{Yb}^{3+}$ emission was anticipated, since the bulk bandgap of $\mathrm{NalnS}_{2}$ lies somewhere between 2.3 and $3.1 \mathrm{eV}$, well above the emitting state of $\mathrm{Yb}^{3+}$. Indeed, incorporation of $\mathrm{Yb}^{3+}$ was confirmed by the appearance of a strong emission band at approximately $990 \mathrm{~nm}$ upon excitation in the host bands. To push the absorption of the nanocrystals more into the visible part of the electromagnetic spectrum, the authors performed cation exchange with different elements $\left(\mathrm{Pb}^{2+}, \mathrm{Cd}^{2+}\right.$, $\left.\mathrm{Ag}^{+}, \mathrm{Cu}^{2+}\right)$. The different chemistry featured by the two cations in the ternary sacrificial material $\left(\mathrm{Na}^{+}\right.$and $\left(n^{3+}\right)$ afforded selective exchange of the alkaline metal in the presence of oleyl amine. Unusual $C d I_{2} S_{4}$, $\mathrm{AgInS}_{2}$ and $\mathrm{Pbln}_{2} \mathrm{~S}_{4}$ polymorphs were produced as a consequence of the retained anion sublattice during cation exchange. The obtained $\mathrm{Pbl} \mathrm{n}_{2} \mathrm{~S}_{4}$ polymorph was not a previously reported structure, which was retrieved from the analysis of its $\mathrm{X}$-ray diffraction pattern: to reproduce the pattern, one $\mathrm{Pb}^{2+}$ substituted every two $\mathrm{Na}^{+}$in the parent $\mathrm{NalnS}_{2}$ structure (Figure 15A).

The best results in terms of host sensitization of the lanthanide luminescence were obtained with $\mathrm{Pbln}_{2} \mathrm{~S}_{4}: \mathrm{Yb}^{3+}$. The retention of $\mathrm{Yb}^{3+}$ (along with $\mathrm{In}^{3+}$ ) in the lattice was ensured by the poor affinity towards the cations featured by oleyl amine molecules in the exchange solution. The final LnSNC had a narrower bandgap compared to $\mathrm{NalnS}_{2}$ nanocrystals, which allowed for better harvesting of visible light (Figure 15B). Interestingly, the authors did not provide a definitive interpretation of the sensitization mechanism. Nonetheless, they suggested a charge-transfer-mediated mechanism or the presence of trap states (also present in parent NalnS 2 nanocrystals) capable of effectively bridging the excited states of the host and the lanthanide ion (Figure 15C).

\subsection{Case study III. CsPbX $3: \mathrm{Yb}^{3+}$ (and other halide perovskites)}

6.3.1. Lead-based perovskites. In the past five years, halide perovskites have been an incredibly hot research topic. In particular all-inorganic halide perovskites are being extensively investigated, with the $\mathrm{CsPbX}_{3}$ family (where $\mathrm{X}=\mathrm{Cl}, \mathrm{Br}, \mathrm{I}$ ) displaying the best performances in terms of light absorption and emission efficiency. These materials have the typical stoichiometry of perovskites $\left(A B X_{3}\right)$ and their structure comprises three ion sites: A-site, B-site and X-site.

A

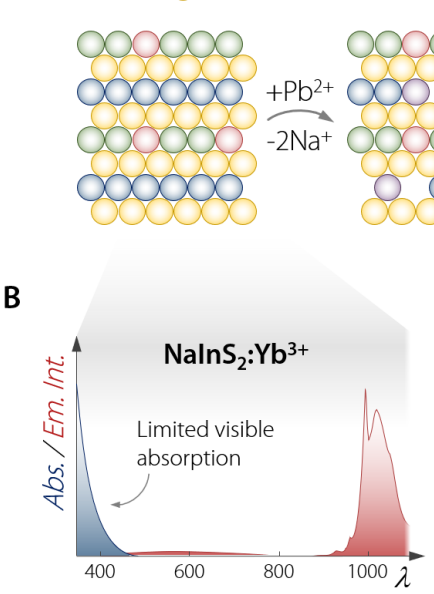

\section{$\mathrm{Yb}^{3+} \mathrm{OPb} \mathrm{Pb}^{2+}$}
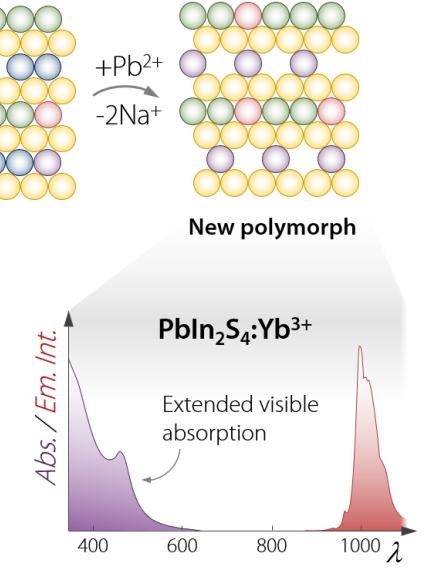

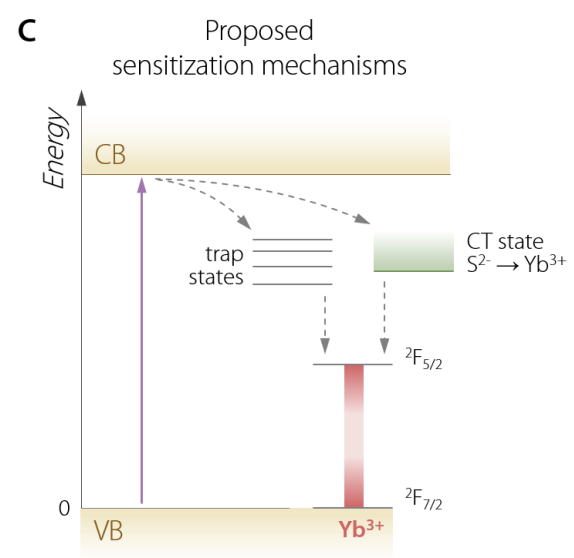

Figure 15. How to leverage cation exchange for the preparation of LnSNCs. A: graphical representation of the cation exchange process, where each $\mathrm{Pb}^{2+}$ ion substitutes two $\mathrm{Na}^{+}$ions, affording the transformation from $\mathrm{NaInS}_{2}: \mathrm{Yb}^{3+}$ to $\mathrm{Pbln}_{2} \mathrm{~S}_{4}: \mathrm{Yb}^{3+}$. B: Absorption and emission spectra before and after cation exchange. The absorption extends to longer wavelength, covering a good part of the visible spectrum. C: Proposed sensitization mechanisms in $\mathrm{PbIn}_{2} \mathrm{~S}_{4}: \mathrm{Yb}^{3+}$. The suggested paths involved either trap states in the nanocrystal or a host-to-lanthanide charge transfer state. Adapted with permission from Ref. ${ }^{50}$. Copyright 2017 American Chemical Society. 
In $\mathrm{CsPbX}_{3}, \mathrm{~Pb}^{2+}$ occupies the octahedral B-site that is favorable for the substitutional doping of $\mathrm{Ln}^{3+}$

(Figure 16A). In particular, $\mathrm{Ln}^{3+}$ doping proceeds via substitution of three $\mathrm{Pb}^{2+}$ by two $\mathrm{Ln}^{3+}$ and the introduction of a cation vacancy $\left(\mathrm{V}_{\mathrm{Pb}}\right) .51,52,55,84,221,265$ The slight mismatch in ionic radius and oxidation state between $\mathrm{Pb}^{2+}$ and $\mathrm{Ln}^{3+}$ results in distortions of the $\mathrm{PbX}_{6}$ octahedra, as well as the appearance of ionic vacancies for each three $\mathrm{Pb}^{2+}$ ions being replaced. $\mathrm{CsPbCl}_{3}$ is the halide perovskite that affords singly doped LnSNCs with the highest emission efficiency.

A

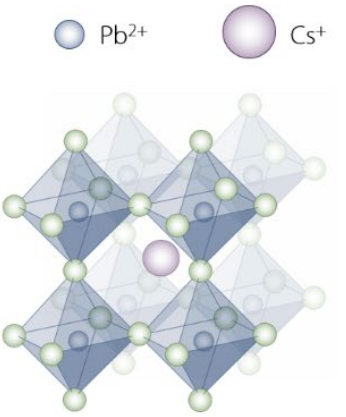

$\operatorname{Ln}^{3+}$

$\mathrm{O} \times \mathrm{vpb}_{\mathrm{Pb}}$
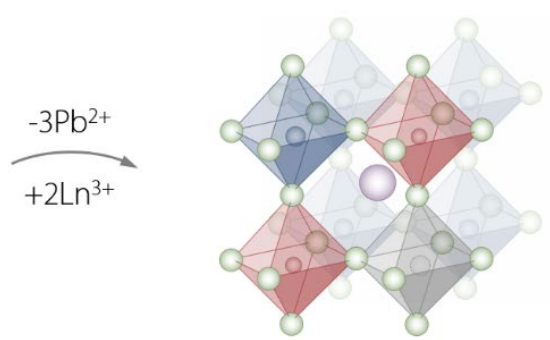

B
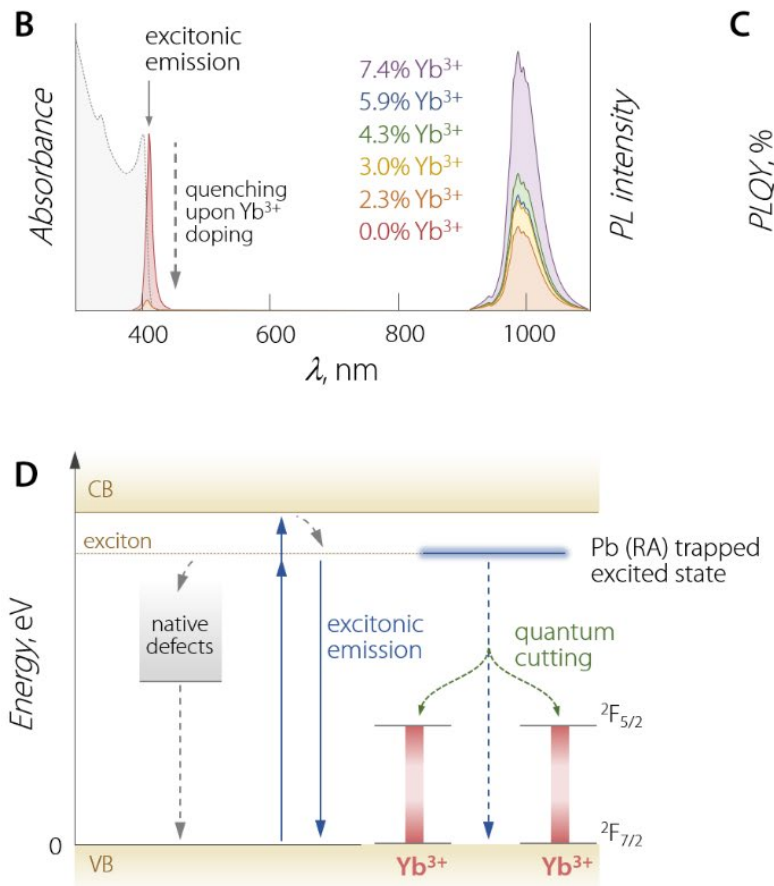

C

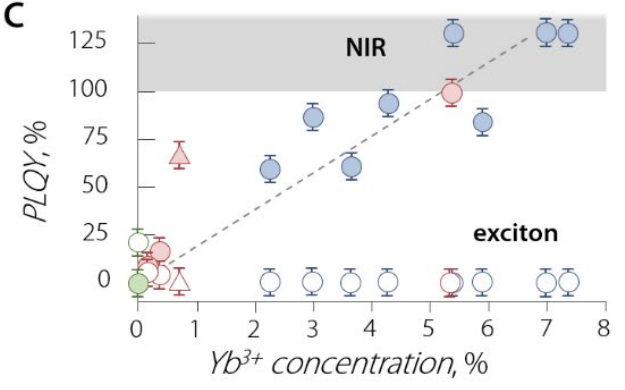

E

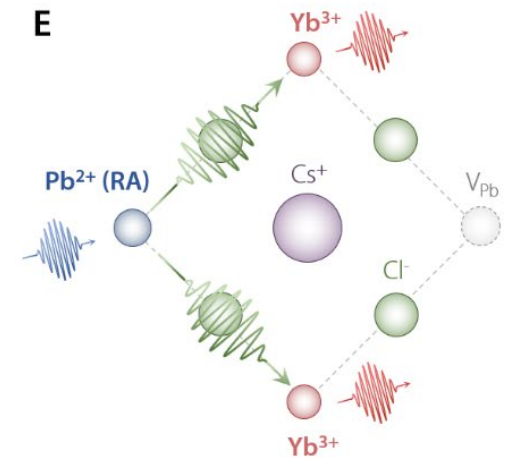

Figure 16. Doping lanthanides in halide perovskites. A: Representation of the typical structure of a $\mathrm{CsPbX}_{3}$ halide perovskite, with the characteristic $\mathrm{PbX}_{6}$ octahedra. Doping with $\mathrm{Ln}^{3+}$ ions proceed through the incorporation of 2 $\mathrm{Ln}^{3+}$ for each $3 \mathrm{~Pb}^{2+}$ and the creation of a $\mathrm{Pb}^{2+}$ vacancy $\left(\mathrm{V}_{\mathrm{Pb}}\right)$. B: Absorption spectrum of undoped $\mathrm{CsPbCl}_{3} \mathrm{SNCS}$ (gray spectrum) and emission spectra of undoped and $\mathrm{Yb}^{3+}$-doped SNCs (colored spectra). The absorption spectrum does not change appreciably upon incorporating $\mathrm{Yb}^{3+}$ ions. C: excitonic emission (empty symbols) is quenched and NIR emission (filled symbols) almost linearly increase with increasing $\mathrm{Yb}^{3+}$ doping percentage. $\mathbf{D}$ : Proposed sensitization mechanisms in $\mathrm{CsPbCl}_{3}: \mathrm{Yb}^{3+}$ as described in Ref. ${ }^{117}$. Non-radiative mechanisms are indicated by dashed lines, excitonic emission is indicated by a solid downwards blue line and $\mathrm{Yb}^{3+}$ emission by gradient red rectangles. E: Simplified representation of a "right angle" (RA) $\mathrm{Yb}^{3+}-\mathrm{VPb}_{\mathrm{Pb}}-\mathrm{Yb}^{3+}$ complex defect, along with the excitation (blue arrow), energy transfer (green arrows) and emission (red arrows) processes. $\mathbf{B}$ and $\mathbf{C}$ are adapted with permission from Ref. ${ }^{84}$. Copyright 2018 American Chemical Society. $\mathbf{E}$ is adapted with permission from Ref. ${ }^{117}$. Copyright 2018 American Chemical Society. 
This is the result of a particularly fortunate series of coincidences. Although from a structural standpoint further investigations are required to thoroughly understand the behavior of halide perovskites, studies point at a beneficial role played by pre-existing defects on the incorporation of $\mathrm{Ln}^{3+} .{ }^{103}$ Indeed, one of the most common type of defect in halide perovskites are halide vacancies, which are also known to reduce the optical performance of the material. ${ }^{266}$ Since the incorporation of $\mathrm{Ln}^{3+}$ proceeds via the creation of such defects, the pre-existence of halide vacancies should lower the energy required to dope $\mathrm{Ln}^{3+}$ ions as impurities in the lattice. From the spectroscopy standpoint, halide perovskites are exceptionally tolerant to defects, with their optical properties being minimally influenced by introduction of impurities. ${ }^{267,268}$ This defect tolerance is guaranteed by the lack of interbandgap states introduced by the impurities and the negligible change of the CB density of states (DOS) they bring about. ${ }^{117,269}$ To that end, as observed by Gamelin and co-workers, ${ }^{84}$ the absorption and excitonic emission of $\mathrm{CsPbCl}_{3} \mathrm{SNCs}$ does not change sizably in terms of spectral shape and position upon incorporation of increasing amounts of $\mathrm{Yb}^{3+}$. However, this host material can effectively funnel the absorbed energy to the doped $\mathrm{Ln}^{3+}$ ions, ultimately resulting in the sensitization of $\mathrm{Ln}^{3+}$ emission and quenching of the excitonic emission (Figure 16B, C). Interestingly, the calculated PLQY for the $\mathrm{Yb}^{3+}$ (NIR) emission almost linearly correlated with the lanthanide doping percentage, surpassing 100\% above 5\% of doped $\mathrm{Yb}^{3+}$. The combination of high absorption efficiency and long lifetime of $\mathrm{Yb}^{3+}$ in the SNC $(>2$ ms) yielded emission saturation already at moderate excitation powers. Indeed, upon reducing the power of the excitation source, a maximum PLQY of 170\% was observed for the sample doped with 5.2\% $\mathrm{Yb}^{3+}$. Those high values could only be explained invoking a sensitization process of quantum cutting, meaning that two low-energy photons are emitted following absorption of a single higher-energy photon. With the help of low-temperature spectroscopic measurements on $\mathrm{La}^{3+}$-doped SNCs, the authors suggested that the introduction of trivalent $\mathrm{RE}$ ions in $\mathrm{CsPbCl}_{3}$ results in a $\mathrm{RE}-\mathrm{V}_{\mathrm{Pb}}-\mathrm{RE}$ defect complex, which generates a shallow e-trap state immediately below the CB involved in the quantum cutting process. Indirect proof of quantum cutting was found by preparing $\mathrm{CsPb}\left(\mathrm{Cl}_{x}, \mathrm{Br}_{1-x}\right)_{3}: \mathrm{Yb}^{3+} \mathrm{SNCs}$ and monitoring $\mathrm{Yb}^{3+}$ emission intensity as a function of the bandgap. ${ }^{270}$ Starting from $\mathrm{CsPbCl}_{3}: \mathrm{Yb}^{3+} \mathrm{SNCs}$, the bandgap was controlled by gradual exchange of $\mathrm{Cl}^{-}$by $\mathrm{Br}$, thus spanning the 3.06-2.4 eV range. The PLQY of $\mathrm{Yb}^{3+}$ plunged upon reaching the critical value of $2.534 \mathrm{eV}$, which is exactly double the energy difference between ${ }^{2} \mathrm{~F}_{5 / 2}{ }^{2} \mathrm{~F}_{7 / 2}$ states of the lanthanide. Later, Ågren and collaborators validated through theoretical calculations the presence of quantum cutting in $\mathrm{CsPbCl}_{3}: \mathrm{Yb}^{3+}$, dismissing the involvement of defect-related interbandgap states. ${ }^{117}$ They provided evidence for the central role played by a trapped excited state centered at the $\mathrm{Pb}^{2+}$ that is part of a "right angle" (RA) $\mathrm{Yb}^{3+}-\mathrm{VPb}^{-} \mathrm{Yb}^{3+}$ complex (Figure 16D, E). Calculations showed that this is the more energetically favorable type of defect in $\mathrm{CsPbCl}_{3}: \mathrm{Yb}^{3+}$ and that it contributes to a larger extent to the DOS near the conduction band, because of the higher degree of distortion of this $\mathrm{PbCl}_{6}$ octahedron. Hence the excited state localized at $\mathrm{Pb}(\mathrm{RA})$ is effectively populated via photoexcitation and the natural proximity of this ion to two $\mathrm{Yb}^{3+}$ facilitates the quantum cutting mechanism.

The abovementioned LnSNCs were obtained via a hot-injection method, relying on statistical mixing. Therein, the chosen precursors were acetates of the respective metal ions and chlorotrimethylsilane (TMS-Cl) was used as the anion source. This method has the advantage of employing acetates instead of the more commonly used halide salts of the metal ions, the former being more soluble in the organic solvents utilized for the synthesis of halide perovskites SNCs. However, cation exchange offers an alternative route to dope $\mathrm{Ln}^{3+}$ in halide perovskite SNCs, as demonstrated by Mir, Nag and co-workers. ${ }^{221}$ Indeed, the authors reported on room temperature exchange of $\mathrm{Mn}^{2+}$ and $\mathrm{Yb}^{3+}$, which was achieved within one minute. It is important to note that, irrespective of the approach, the incorporation efficiency (fraction of incorporated $\mathrm{Ln}^{3+} \mathrm{vs}$. nominal $\mathrm{Ln}^{3+}$ content) remains usually below $10 \%$. 
The group of Song further expanded the scope of $\mathrm{Ln}^{3+}$ doping of halide perovskites SNCs, exploring the incorporation of multiple lanthanides. ${ }^{52,265}$ Combination of $\mathrm{Ce}^{3+}-\mathrm{Yb}^{3+}$ in $\mathrm{CsPbCl}_{1.5} \mathrm{Br}_{1.5}{ }^{265}$ and $\mathrm{Pr}^{3+}-\mathrm{Yb}^{3+}$ or $\mathrm{Ce}^{3+}-\mathrm{Pr}^{3+}-\mathrm{Yb}^{3+}$ in $\mathrm{CsPbClBr}_{2}^{52}$ were found to yield the best quantum cutting efficiency among the hostdopants pairs investigated. The authors explained this performance considering the involvement of $\mathrm{Ce}^{3+}$ $5 \mathrm{~d}$ and/or $\mathrm{Pr}^{3+}{ }^{3} \mathrm{P}_{1-0}$ as bridging states for the quantum cutting mechanism to occur. Particularly in the triply doped SNCs, the role of $\mathrm{Ce}^{3+}$ was explained to be functional in terms of reducing the energy mismatch between the host material bandgap and excited $\mathrm{Pr}^{3+}$ states, while also directly feeding $\mathrm{Yb}^{3+}$ via quantum cutting. Overall, a maximum PLQY of $173 \%$ was achieved with $\mathrm{Ce}^{3+}-\mathrm{Pr}^{3+}-\mathrm{Yb}^{3+}$-doped $\mathrm{CsPbClBr}$. These LnSNCs were used to deposit a light conversion layer on commercial CIGS and Si-solar cells via liquid-phase deposition. The result was a $20 \%$ increase of the photon conversion efficiency (PCE) of both types of cells.

6.3.2. Lead-free perovskites. Aside from Pb-based perovskites, several groups are exploring less toxic, heavy-metal-free alternatives. For instance, $\mathrm{Cs}_{3} \mathrm{Bi}_{2} \mathrm{Br}_{9} \mathrm{SNCs}$ doped with $\mathrm{Eu}^{3+}$ were recently prepared and used for the detection of copper ions in aqueous environments, ${ }^{271}$ whilst the same material doped with $\mathrm{Sm}^{3+}$ was proposed for LED applications. ${ }^{272}$ However, the class of lead-free perovskites more explored as of late for $\mathrm{Ln}^{3+}$ doping is the one of halide double perovskites (HDPs). In a recent study, Xia and coworkers systematically investigated the incorporation of $\mathrm{Tb}^{3+}$ in $\mathrm{SNCS}$ of $\mathrm{Cs}_{2} \mathrm{AglnCl}_{6}$ doped with $\mathrm{Bi}^{3+273}$ The authors observed $\mathrm{Tb}^{3+}$ doping efficiencies between 25 and 75\% (maximum $\mathrm{Tb}^{3+}$ amount doped of 20\%), with overall quantum yields (trapped excitonic emission $+\mathrm{Tb}^{3+}$ emission) between 6.6 and 10.1\%. Doping with $\mathrm{Bi}^{3+}$ was necessary to observe $\mathrm{Tb}^{3+}$ sensitized emission upon UV excitation, since $\mathrm{Bi}^{3+}$ promotes the formation of a localized STEs that efficiently feed the emitting state of the lanthanide ion. Theoretical calculations showed that the incorporation of $\mathrm{Tb}^{3+}$ within the lattice occurs substitutionally rather than interstitially, with the most energetically favorable site being the octahedron (B-site) occupied by $\mathrm{In}^{3+}$. Other examples of SNCs composed of $\mathrm{Ln}^{3+}$-doped HDPs are $\mathrm{CS}_{2} \mathrm{AgBiX}_{6}: \mathrm{Yb}^{3+}(\mathrm{X}=\mathrm{Cl}$, $\mathrm{Br})_{1}^{274} \mathrm{Cs}_{2} \mathrm{AgInCl}_{6}: \mathrm{Yb}^{3+}-\mathrm{Er}^{3+},{ }_{1}^{275} \mathrm{Cs}_{2} \mathrm{AgInCl} 6: \mathrm{Yb}^{3+},{ }^{276}$ and $\mathrm{Cs}_{2} \mathrm{Ag}_{0.4} \mathrm{Na}_{0.6} \mathrm{InCl}_{6}: \mathrm{Bi}^{3+}, \mathrm{Ce}^{3+} .{ }^{277} \mathrm{This}$ blooming of studies on HDPs is expected to continue and lead to exciting results, eventually yielding SNCs with properties comparable to those of lead-based $\mathrm{Ln}^{3+}$-doped halide perovskites.

6.3.3. Lanthanide-based perovskites. Recently, Huang et al. ${ }^{112}$ reported the preparation of $\mathrm{CsEuCl}_{3}$ SNCs. These crystals are a particular example of Pb-free halide perovskites with a sharp emission peak and strong excitonic absorption. The PLQY was a modest 5.6\% after treatment with 1-butyl-1methylpyridinium chloride (see Section 4.3). However, upon incorporation in a polymethylmethacrylate matrix, the SNCs retained their emission intensity upon prolonged (380 min) exposure to UV light. It is interesting to point out that europium takes its divalent oxidation state in this material, but the contribution of $5 \mathrm{~d}-4 \mathrm{f}$ transitions are not apparent, with the authors of the study leaning towards an excitonic nature of the emission of $\mathrm{CsEuCl}_{3} \mathrm{SNCs}$. This study is an example of how $\mathrm{Eu}^{2+}$ can be obtained starting from $\mathrm{Eu}^{3+}$ salts and relying on the reducing capabilities of specific chemical species (in this case, oleylamine). Notably, $\mathrm{CsEuBr}_{3}$ with a PLQY of 39\% were also prepared, but their synthesis entailed the use of divalent europium precursors (i.e., EuBr 2$).{ }^{111} \mathrm{CsYbl}_{3} \mathrm{SNCs}$ have also been reported, whose excitonic emission is centered at $660 \mathrm{~nm}$ and exhibiting a PLQY of approximately $58 \% .{ }^{278}$ In that case, $\mathrm{Ybl}_{2}$ salt was used as source of ytterbium, which retained its divalent oxidation state in the final material.

\subsection{Case study IV. Alkaline earth sulfides.}

To conclude this parade of representative studies, we have chosen to focus our attention on alkaline earth sulfides (AESs). CaS and SrS feature the divalent alkaline earth in an octahedral $(C N=6)$ coordination environment: a situation that supports substitutional doping of lanthanide ions. As mentioned several times throughout this Review, europium takes its divalent oxidation state when doped in these hosts. Another key consideration is that AESs feature large bandgaps ( $>4 \mathrm{eV}$ ), thus limiting the wavelength range exploitable for host-sensitization. Consequently, these hosts have been 
used mainly to prepare LnSNCs doped with lanthanides featuring 4f-5d transitions, i.e., $\mathrm{Ce}^{3+}$ and $\mathrm{Eu}^{2+} .{ }^{107}$ Occasionally, co-doping with other $\mathrm{Ln}^{3+}$ ions is performed, which allows for emission tuning as well as persistent luminescence. ${ }^{108,109,126,279}$

Zhao et al. reported the synthesis of LnSNCs based on CaS and SrS via the thermal decomposition of single-source precursors of the host metals and lanthanides (Figure 17A, C). ${ }^{107}$ The authors demonstrated the possibility to pursue both growth doping and nucleation doping strategies to achieve effective incorporation of the lanthanide ions. Diisopropyldithiocarbamate (DDTC) complexes of $\mathrm{Ca}^{2+}$ and $\mathrm{Sr}^{2+}$ were used as host precursors, while tetrakis lanthanide complexes (with general formula $\mathrm{LnL}_{4}$ ) of different mono- and dialkyl carbamates were tested. SrS:Ce ${ }^{3+}$ SNCs were obtained by simple statistical mixing (co-injection in oleylamine at $250^{\circ} \mathrm{C}$ ) of $\mathrm{Sr}(\mathrm{DDTC})_{2}$ and $\mathrm{Ce}(\mathrm{DDTC})_{4}$. However, overgrowth of a further shell of host material atop of the LnSNC resulted in a better incorporation of $\mathrm{Ce}^{3+}$ ions adsorbed on the surface of the nanocrystals, following the growth doping strategy (Figure 17A). The excitation spectrum of the LnSNCs shows the presence of $4 \mathrm{f}-5 \mathrm{~d}$ transitions of $\mathrm{Ce}^{3+}$ (centered at $430 \mathrm{~nm}$ ), as well as the contribution of the host below $320 \mathrm{~nm}$ (Figure 17B). This is an indication of the possibility of the material to absorb the excitation light and transfer it to the dopants. Moreover, upon increasing the content of $\mathrm{Ce}^{3+}$ from 0.1 to $10 \%$, the emission peak maximum shifts at longer wavelength due to selfreabsorption mechanisms, thus affording color tuning. The authors prepared CaS:Ce ${ }^{3+} \mathrm{LnSNCs}$ using the same approach, but the use of Eu(DDTC) ${ }_{4}$ did not allow for effective incorporation of $\mathrm{Eu}^{2+}$ in the structure. The different behavior of the two lanthanides was ascribed to the different decomposition temperatures featured by $\mathrm{Ce}(\mathrm{DDTC})_{4}$ and $\mathrm{Eu}(\mathrm{DDTC})_{4}: 150$ and $260^{\circ} \mathrm{C}$ respectively, the latter being higher than the reaction temperature of $250^{\circ} \mathrm{C}$. To achieve incorporation of $\mathrm{Eu}^{2+}$ in the SNCs, two different approaches were pursued. Europium complexes with lower decomposition temperatures (based on monoalkyldithiocarbamates) were used instead of Eu(DDTC) 4 to prepare LnSNCs via the same method reported for $\mathrm{Ce}^{3+}$-doped $\mathrm{CaS}$ and $\mathrm{SrS}$. The shell of host material was overgrown at lower temperatures $\left(200^{\circ} \mathrm{C}\right.$ ), since at $250^{\circ} \mathrm{C}$ the incorporated $\mathrm{Eu}^{2+}$ showed the tendency to migrate towards the surface of the SNCs, where it is more exposed to quenching (see Section 4). The other approach proposed by the authors is a nucleation doping protocol and it leverages the abovementioned thermal diffusion of $\mathrm{Eu}^{2+}$ along with the different reactivity exhibited by DDTC precursors of europium and alkaline earths. Small EuS SNCs were initially prepared at high temperature $\left(320^{\circ} \mathrm{C}\right)$ and used as seeds to grow a shell of SrS at $250^{\circ} \mathrm{C}$ (Figure 17C). Upon growing several shells of material, the $\mathrm{Eu}^{2+}$ ions in the core started diffusing through the SNC volume. This migration was followed spectroscopically: EuS cores are not fluorescent, but upon growing shells of SrS an emission band with increasing intensity appeared around $610 \mathrm{~nm}$ (Figure 17D).

Recently, Zheng, Chen and co-worker reported CaS:Ce ${ }^{3+}, \mathrm{Ln}^{3+} \mathrm{LnSNCs}\left(\mathrm{Ln}^{3+}=\mathrm{Er}^{3+} \mathrm{Or} \mathrm{Nd}^{3+}\right)$ which can emit within the NIR under UV-blue excitation..$^{109}$ Upon mixing acetate metal salts and the sulfur source $\left(N, N^{\prime}-\right.$ diphenyl thiourea) in the presence of coordinating molecules (oleic acid, oleylamine and trioctylamine), $\mathrm{Ln}^{3+}$-doped CaS SNCs were obtained after $60 \mathrm{~min}$ at $320^{\circ} \mathrm{C}$ (Figure 17E). Although host-sensitization of the $\mathrm{Ln}^{3+}$ emission was observed, once more the allowed transition of $\mathrm{Ce}^{3+}$ was exploited to harvest the excitation light and funnel the energy to the other lanthanide ion doped in the structure. In $\mathrm{CaS}: 0.07 \% \mathrm{Ce}^{3+}, \mathrm{x} \% \mathrm{Er}^{3+}$, the most intense emission was observed for $0.5 \%$ of $\mathrm{Er}^{3+}$ (Figure 17F), with a calculated cerium-to-erbium ET efficiency of $85.1 \%$ and absolute NIR PLQY of $(9.3 \pm 0.4) \%$. The lifetime of $\mathrm{Er}^{3+}$ and $\mathrm{Ce}^{3+}$ emissions both decreased with increasing $\mathrm{Er}^{3+}$ concentration, owing to increased $\mathrm{Er}^{3+}-$ $\mathrm{Er}^{3+}$ and $\mathrm{Ce}^{3+}-\mathrm{Er}^{3+} \mathrm{ET}$ processes, respectively (Figure 17G). The LnSNCs were finally coated with phospholipids, transferred to water, and used for the detection of $\mathrm{H}_{2} \mathrm{O}_{2}$ and the $\mathrm{H}_{2} \mathrm{O}_{2}$-producing disease marker xanthine. 

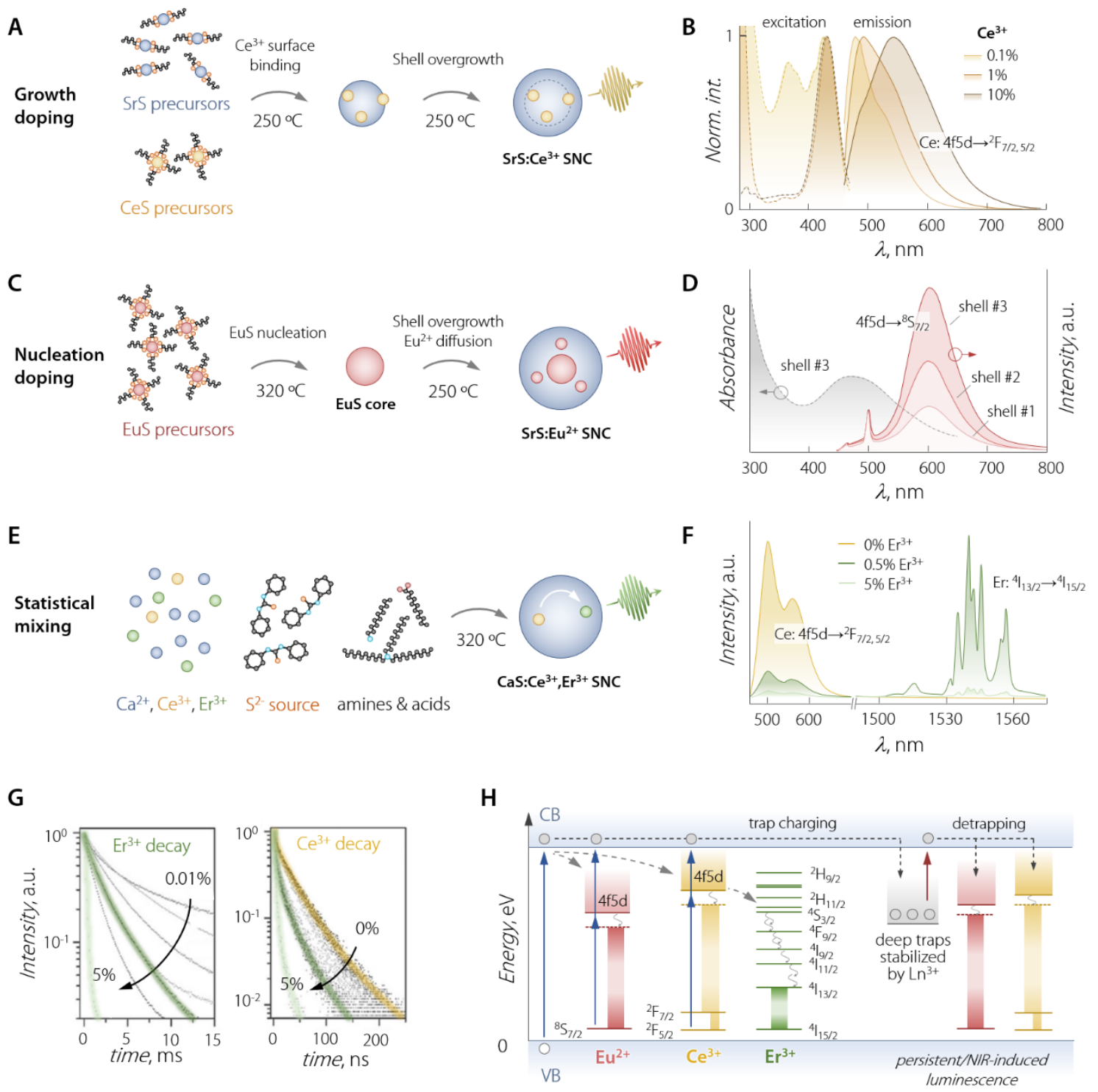

Figure 17. Alkaline earth sulfides as versatile hosts for lanthanide doping. Schemes for the growth doping (A) and nucleation doping $(\mathbf{C})$ strategies developed by Zhao et al. for the preparation of LnSNCs based on AESs. B: excitation (monitored at the emission maxima) and emission $\left(\lambda_{e x}=430 \mathrm{~nm}\right.$ ) spectra of SrS doped with different amounts of $\mathrm{Ce}^{3+}$. D: emission spectra (red solid lines, $\lambda_{e x}=430 \mathrm{~nm}$ ) of SrS:Eu+ LnSNCs after growth of different number of shells of SrS, along with the absorption spectrum of the system after the growth of the third shell (gray dashed line). $\mathbf{E}$ : scheme of the synthesis method for the preparation of NIR-emitting CaS-based LnSNCs. F: emission spectra $\left(\lambda_{e x}=450 \mathrm{~nm}\right)$ of CaS co-doped with $\mathrm{Ce}^{3+}(0.07 \%)$ and different amounts of $\mathrm{Er}^{3+}$. G: photoluminescence decay curves recorded for samples doped with different $\mathrm{Er}^{3+}$ amounts monitoring $\mathrm{E}^{\mathrm{r}+{ }^{4} \mathrm{I}_{13 / 2}} \rightarrow{ }^{4} \mathrm{I}_{15 / 2}$ (right) and $\mathrm{Ce}^{3+}$ $4 \mathrm{f5d} \rightarrow{ }^{2} \mathrm{~F}_{7 / 2,5 / 2}$ (left). $\mathbf{H}$ : Summary of the proposed electronic transitions and emission mechanisms occurring in AES-based LnSNCs. A-D: adapted with permission from Ref. ${ }^{107}$. Copyright 2014 American Chemical Society. E-G: adapted with permission from Ref. ${ }^{109}$. Copyright 2019 Wiley-VCH.

The versatility of AESs as host materials for $\mathrm{Ln}^{3+}$ is further demonstrated by the possibility to prepare long-lasting and/or NIR-triggered nanophosphors using them. ${ }^{126}$ This is the case in particular of $\mathrm{Sm}^{3+}$ $\mathrm{Ce}^{3+}$ and $\mathrm{Sm}^{3+}-\mathrm{Eu}^{2+}$ co-doped AES LnSNCs (Figure 17H). In those nanophosphors, $\mathrm{Sm}^{3+}$ lowers the energy of endogenous trap states of the material, which in turn act as electron reservoirs after being charged with UV light. This "pre-charging" promotes electrons from the valence band of the host and/or from the $4 \mathrm{f}$ ground state of $\mathrm{Ce}^{3+}$ and $\mathrm{Eu}^{2+}$ to the conduction band. Part of the electrons are trapped in 
the interbandgap states, from where they can be re-promoted to the CB both via thermal activation and NIR excitation. Finally, de-trapped electrons can populate the $5 \mathrm{~d}$ states of $\mathrm{Eu}^{2+}$ and $\mathrm{Ce}^{3+}$ and finally relax back to the lanthanides' ground state with emission of light. This process was also observed by substituting $\mathrm{Sm}^{3+}$ by $\mathrm{Dy}^{3+108}$ and it was shown to be enhanced by addition of $\mathrm{Mn}^{2+}$ in the structure. ${ }^{106,279}$

\section{Applications}

The interest around a class of materials is directly proportional to their applicability in technologically relevant fields. This is also the case for LnSNCs with photoluminescence properties, whose light absorption and emission capabilities make them foreseeable candidates in optoelectronics, sensing, and in the biomedical context. Below, we summarize the most recent advancements in the application of LnSNCs so far discussed in this Review. It is important to point out that the use of LnSNCs based on oxides (above all, $\mathrm{TiO}_{2}$ and $\mathrm{ZnO}$ ) has been reviewed in numerous instances. ${ }^{280-283}$ Moreover, arguably the most widespread use of $\mathrm{Ln}^{3+}$-doped $\mathrm{TiO}_{2}$ and $\mathrm{ZnO}$ nanomaterials is in photocatalysis, where the photoluminescence properties of lanthanides (and even less so their host-sensitized emission) play a marginal role. However, LnSNCs based on these hosts have also been proposed as light converters to enhance the performances of solar cells, ${ }^{130,284,285}$ for the preparation of UV/blue excited light emitting devices, ${ }^{283}$ as well as nanoprobes in biological environments, ${ }^{286}$ optical contrast agents, ${ }^{287}$ and anticounterfeiting tags. ${ }^{288}$ Given the fact that these materials have been known for some time now, we decided to focus our attention instead on the proposed applications for photoluminescent LnSNCs developed more recently, such as those based on halide perovskites and AESs.

\subsection{Photovoltaics.}

Creutz et al. in their study (Section 6.2) suggested that one of the foreseeable applications of $\mathrm{Pbln} \mathrm{S}_{2}: \mathrm{Yb}^{3+} \mathrm{LnSNCS}$ could be as light harvester in photovoltaic devices, owing to the good overlap of LnSNCs' absorption with the solar spectrum and the emission centered at $980 \mathrm{~nm}$ utterly falling in correspondence of the absorption edge of silicon solar cells. ${ }^{50}$ Indeed, the field of photovoltaics is the one where LnSNCs are envisaged to have a substantial impact, and $\mathrm{Ln}^{3+}$-doped halide perovskites are a uniquely coveted class of materials in that regard. These materials are heavily investigated in the context of the technology of perovskite solar cells, which makes use of perovskites as the semiconductor material wherein electron-hole pairs are photogenerated. ${ }^{289}$ Doping with lanthanides has proven beneficial both in terms of increasing the efficiency and the stability (their Achille's heel) of perovskite solar cells..$^{290,291}$ Photoluminescence LnSNCs based on halide perovskites can be used instead to improve the performance of commercially available Si-cells ${ }^{265}$ and thin-film cells (e.g., Culn ${ }_{1-x} \mathrm{Ga}_{x} \mathrm{Se}_{2}$ - CIGS). ${ }^{52}$ In those photovoltaic devices, the phenomenon of thermalization (Figure 18A) - occurring after absorption of high-energy photons (UV) - results in loss of power conversion efficiency (PCE) of the device.292 Quantum cutting materials could provide an answer to this issue by converting a single UV photons into two NIR photons, which can in turn be more effectively absorbed by the material where charge separation occurs. As we have discussed in detail in Section 6.3, many perovskite-based LnSNCs doped with Yb ${ }^{3+}$ (and/or additionally co-doped with other $\mathrm{Ln}^{3+}$ ) feature this quantum cutting capability. ${ }^{84} \mathrm{Zhou}$ et al. used CsPbCl ${ }_{1.5} \mathrm{Br}_{1.5}: \mathrm{Yb}^{3+}, \mathrm{Ce}^{3+}$ LnSNCs with a NIR PLQY of $119 \%$ (total PLQY of 146\%) to cover the surface of a commercial Si-cell via liquid-phase deposition. ${ }^{265}$ The authors controlled the thickness of the deposited film between 60 and $770 \mathrm{~nm}$, observing the highest improvement (PCE from 18.1 to 21.5\%) with a film thickness of $230 \mathrm{~nm}$. The same authors went on to test the efficacy of this approach using multi-doped LnSNCs $\left(\mathrm{Yb}^{3+}\right.$ and $\left.\mathrm{Dy}^{3+}, \mathrm{Tb}^{3+}, \mathrm{Nd}^{3+}, \mathrm{Pr}^{3+}, \mathrm{Ce}^{3+}, \mathrm{Ce}^{3+} / \mathrm{Pr}^{3+}\right)$ with generic host composition $\mathrm{CsPbCl} \mathrm{Br}_{x} \mathrm{l}_{3-x-y .} .{ }^{2 .}$ Triply doped $\mathrm{CsPbClBr} 2 \mathrm{Yb}^{3+}, \mathrm{Ce}^{3+}, \mathrm{Pr}^{3+} \mathrm{LnSNCs}$ showed the highest PLQY of $173 \%$ under $365 \mathrm{~nm}$ excitation and were used to coat Si- and CIGS-cells. For both devices, a relative PCE enhancement of roughly $20 \%$ was observed. Interestingly, the authors utilized the CIGS solar cell to recharge a mobile phone, observing a shortening of the recharging time of $30 \mathrm{~min}$ (from 180 to $150 \mathrm{~min}$ ) for the solar cell coated with LnSNCs. 
A
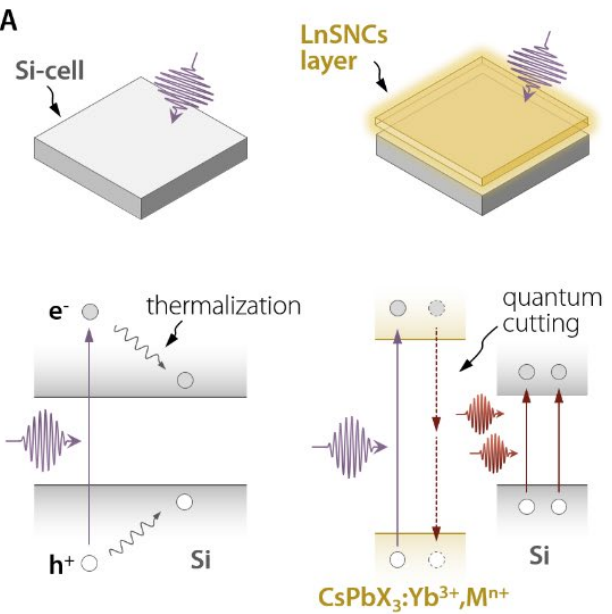

B

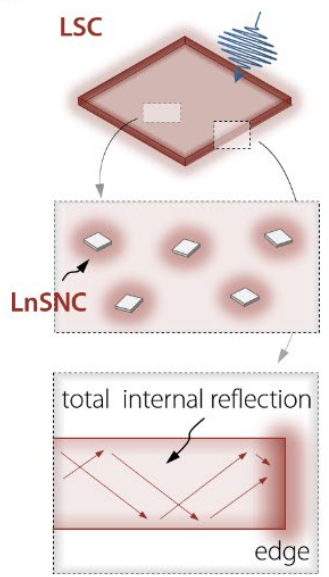

C
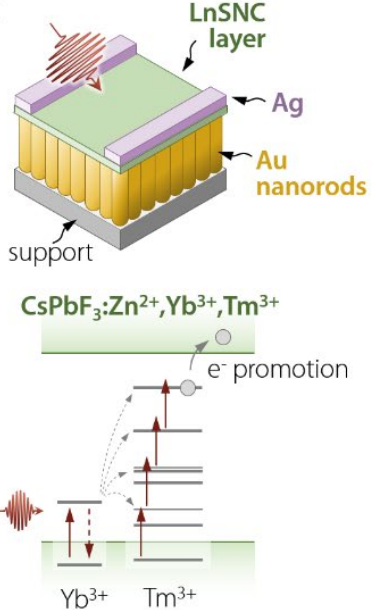

D
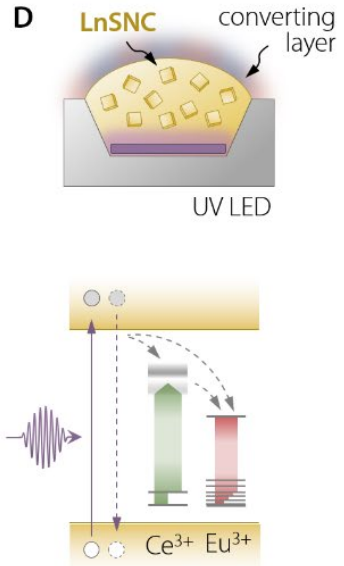

$\mathrm{CsPbCl}_{3}: \mathrm{Ce}^{3+}, \mathrm{Eu}^{3+}$
$\mathrm{E}$
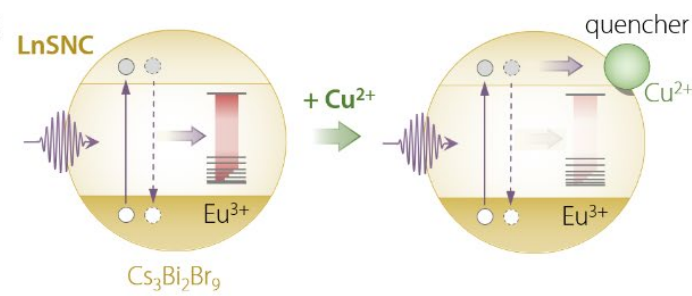

G

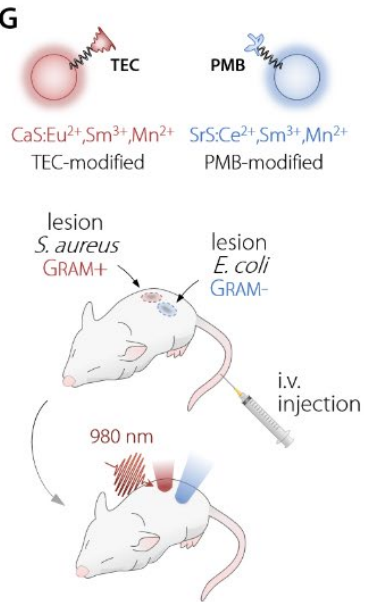

Figure 18. Selection of LnSNCs applications. A: operation principle of a photovoltaic silicon cell covered with a converting layer of $\mathrm{CsPbX}_{3} \mathrm{SNCs}$ doped with $\mathrm{Yb}^{3+}$ and other ionic species $\left(\mathrm{M}^{\mathrm{n}}\right)$. Note how the photons whose energy goes partially lost via thermalization are more efficiently harnessed thanks to quantum cutting. B: working principle of a luminescent solar concentrator based on LnSNCs. C: scheme of a narrow-band NIR photodetector based on upconverting $\mathrm{CsPbF}_{3}: \mathrm{Zn}^{2+}, \mathrm{Yb}^{3+}, \mathrm{Tm}^{3+} \mathrm{LnSNCs}$ (top) and charge generation mechanism proposed by the authors (bottom). Adapted with permission from Ref. ${ }^{293}$. Copyright 2020 Elsevier. D: scheme of a wLED composed of a UV LED coated with a polymeric dispersion of LnSNCs acting as converting layer (top) and the partial energy level scheme of $\mathrm{CsPbCl}_{3}: \mathrm{Ce}^{3+} \mathrm{Eu}^{3+}$ LnSNCs used in Ref. ${ }^{51}$ for the preparation of such device. E: scheme of the functioning mechanism of the $\mathrm{Cu}^{2+}$ sensor based on $\mathrm{Cs}_{3} \mathrm{Bi}_{2} \mathrm{Br}_{9}: \mathrm{Eu}^{3+}$ developed in Ref. ${ }^{271}$. F: scheme of the processes involved in the quenching of the luminescence of CaS:Ce ${ }^{3+}, \mathrm{Er}^{3+}$ LnSNCs used as xanthine sensor in Ref. ${ }^{109}$. G: representation of CaS- and SrS-based LnSNCs modified with TEC (Gram + specific) and PMB (Gram - specific) (top) used for selective imaging of infected lesions upon intravenous injection (Ref. ${ }^{279}$ ).

Another use of perovskites LnSNCs that has been showcased in the field of photovoltaics is the preparation of luminescent solar concentrators (LSCS). ${ }^{294-296}$ This technology relies on the use of a polymeric dispersion of fluorophores in the form of slabs, on whose edges photovoltaic devices (cells) are attached. ${ }^{297}$ The fluorophores absorb the light of the sun, convert it to light usable by the coupled cells, and concentrate this light at the edges in a waveguide-like fashion by total internal reflection (Figure 18B). A sizeable energy difference between the absorption and the emission of the luminophore is vital to avoid internal losses due to reabsorption within the LSC: this is a condition well satisfied in perovskites LnSNCs doped with $\mathrm{Yb}^{3+}$ (see Figure 16B). Luo et al. first introduced the concept of quantum 
cutting LSC preparing optically transparent LSCs via the deposition of poly(methyl methacrylate) (PMMA)-CsPbCl $3: \mathrm{Yb}^{3+}$ thin films onto borosilicate glass substrates. ${ }^{295}$ The obtained internal efficiency (photons emitted at the edges vs. absorbed) reached a remarkable $118.1 \%$ in a $5 \times 5 \mathrm{~cm}$ slab. This record value was balanced out by the absorption of a limited part of the solar spectrum (owing to the large bandgap of the LnSNCS). Partial substitution of $\mathrm{Cl}^{-}$with $\mathrm{Br}^{-}$allowed reducing the bandgap of the host, thus increasing the overall sun light that can be absorbed by the $\mathrm{Yb}^{3+}$-doped LnSNCs. This extended absorption capability was estimated to lead to an overall (external) efficiency of $9.0 \%$ for a $5 \mathrm{~cm}^{2}$ slab. The use of $\mathrm{CsPb}\left(\mathrm{Cl}_{0.25} \mathrm{Br}_{0.75}\right)_{3}: \mathrm{Yb}^{3+} \mathrm{LnSNCs}$ was shortly after suggested by Cohen et al. for the preparation of two-layer monolithic LSCs, wherein the top and bottom layers contain these LnSNCs and CulnS $2 / Z n S$ SNCs, respectively. ${ }^{296}$ Modelling of this innovative architecture indicated a 19\% improvement of the performance of a single-layer LSC based on CuInS $2 / Z n S$ alone. Very recently, Cai et al. proposed the use of $\mathrm{CsPbCl}_{3}: \mathrm{Yb}^{3+}, \mathrm{Mn}^{2+}$ for the preparation of $\mathrm{LSCs}^{294}$ Upon adjustment of the dopants concentration, emission from the host (approx. $400 \mathrm{~nm}$ ) and the two doped ions (approx. 600 and $1000 \mathrm{~nm}$ ) was achieved under UV excitation, with an overall PLQY of 125.3\%. The authors prepared polydimethylsiloxane slabs with LnSNC concentration of $0.3 \mathrm{wt} \%$ and observed a maximum external efficiency of $9.6 \%$.

Although the use of quantum cutting perovskite-based LnSNCs holds great potential in photovoltaics, as a critical article by Erickson et al. pointed out, intrinsic limitations are imposed by the photoluminescence saturation that is achieved under high solar irradiance. ${ }^{298}$ In the same articles, strategies to overcome this limitation via a rational design of the material and engineering of the photovoltaic devices are also laid out.

\subsection{Photodetectors.}

Aside from photovoltaic applications, halide perovskites were used by Moon et al. to prepare a hybrid SNCs-graphene photodetector $\left(\mathrm{SNC}=\mathrm{CSYbl}_{3}\right)$ in the visible range. ${ }^{278}$ Therein, the strong absorption properties of the perovskite SNCs allowed for efficient photon harvesting up to approximately $700 \mathrm{~nm}$. The authors observed that the photo-generated charge carriers were effectively injected into the graphene before they could recombine within the SNC. The result was a high photoresponsivity of $2.4 \cdot 10^{3} \mathrm{~A} \mathrm{~W}^{-1}$ and an external quantum efficiency (EQE) of $5.8 \cdot 10^{5} \%$ with an incident light power of the order of microwatts $\left(\lambda=515 \mathrm{~nm}\right.$ ). Narrowband NIR photodetectors based on $\mathrm{CsPbF}_{3}$ : $\mathrm{Zn}^{2+}-\mathrm{Yb}^{3+}-\mathrm{Tm}^{3+} / \mathrm{Er}^{3+}$ were also recently proposed (Figure $\mathbf{1 8 C}$ ) ${ }^{293}$ Therein, the process of upconversion initiated by the absorption from $\mathrm{Yb}^{3+}$ eventually leads to the thermal-assisted promotion of electrons in the perovskite's CB. From there, the charge carriers can be extracted to generate an electric signal, yielding a high responsivity of $106 \mathrm{~A} \mathrm{~W}^{-1}$, detectivity of $1.52 \cdot 10^{12}$ Jones, and external quantum efficiency of $135 \%$. The mechanism of free charge generation suggested by the authors entails an electron transfer from the $\mathrm{Ln}^{3+}$ excited $4 \mathrm{f}$ states to the CB of the host, occurring simultaneously with the transfer of an electron in the $\mathrm{VB}$ to the ground state of the $\mathrm{Ln}^{3+}$. We propose that thermally assisted cross-relaxation between $\mathrm{Tm}^{3+}$ and the host material might also explain the generation of free electrons in this system.

\subsection{Light-emitting diodes (LEDs).}

Halide perovskites have also been proposed for white-light emitting device (wLED) preparation, leveraging their UV excitability and good PLQY (Figure 18D). ${ }^{51,277,299-301}$ To that end, Pan et al. coated a 365-nm emitting GaN LED with a layer of PMMA dispersion of $\mathrm{CsPbCl}_{3}: \mathrm{Ce}^{3+}, \mathrm{Eu}^{3+} \mathrm{LnSNCs}$. The combined host-sensitized emission of $\mathrm{Ce}^{3+}$ (broadband, blue-greenish) and $\mathrm{Eu}^{3+}$ (line-like, red) resulted in a cold white light emission with color coordinates $(0.32,0.26)$ and luminous efficiency of $24 \mathrm{~lm} / \mathrm{W} .^{51}$ Interestingly, it was observed that doping with $\mathrm{Ln}^{3+}$ improved the photostability of the LED. The same authors exploited a similar approach, substituting polystyrene to PMMA and used $\mathrm{CsPbCl}_{1.8 \mathrm{Br}} \mathrm{Pl}_{2 .} \mathrm{Ce}^{3+}, \mathrm{Mn}^{2+}$ as LnSNCs with a PLQY of 55\%. ${ }^{299}$ The broadband nature of the emission of both $\mathrm{Ln}^{3+}$ and transition metal 
allowed to achieve color coordinates of $(0.33,0.29)$ and a higher luminance of $51 \mathrm{~lm} / \mathrm{W}$. Although not nanosized, recently crystals of $\mathrm{Cs}_{2} \mathrm{Ag}_{0.4} \mathrm{Na}_{0.6} \mathrm{InCl}_{6}: \mathrm{Ce}^{3+}, \mathrm{Bi}^{3+}$ were also used to prepare wLEDs. ${ }^{277} \mathrm{~A}$ blend of commercially available $\mathrm{BaMgAl}_{10} \mathrm{O}_{17}: \mathrm{Eu}^{2+}$ and the developed perovskite phosphor was used to coat a UVemitting LED, yielding color coordinates of $(0.36,0.33)$ and color rendering index of 95.7 (100 being an ideal light source capable of rendering with complete fidelity the colors of the illuminated object). The use of $\mathrm{Cs}_{2} \mathrm{Agg}_{0.4} \mathrm{Na}_{0.6} \mathrm{lnCl}_{6}: \mathrm{Ce}^{3+}, \mathrm{Bi}^{3+}$ solely resulted instead in a slightly lower color rendering index of 84.1. This latter investigation showcases the continuous efforts towards the development and use of Pb-free perovskites, which is further corroborated by the recent study of Zhu et al, who used $\mathrm{Cs}_{3} \mathrm{Bi}_{2} \mathrm{Br}$ : $\mathrm{Sm}^{3+}$ LnSNCs for the preparation of UV converting layers to be used in wLEDs. ${ }^{272}$ White light was also obtained using a NIR LED as primary emitter, which was covered with a mixture of $\mathrm{CaS} \mathrm{Eu}^{2+}, \mathrm{Sm}^{3+}, \mathrm{Mn}^{2+}$ $\mathrm{CaS}: \mathrm{Ce}^{3+}, \mathrm{Mnn}^{2+}$, and $\mathrm{SrS}: \mathrm{Ce}^{3+}, \mathrm{Sm}^{3+}, \mathrm{Mn}^{2+} \mathrm{LnSNCs}^{106}$ As explained in Section 6.4, the emission of these materials relies on charges trapped after exposure to UV light. Hence, once the reservoir of trapped charges is depleted, no emission under NIR excitation can be observed, overall limiting the applicability of LEDs based on these LnSNCs.

\subsection{Sensing.}

$\mathrm{Cs}_{3} \mathrm{Bi}_{2} \mathrm{Br}_{9}$-based LnSNCs were also doped with $\mathrm{Eu}^{3+}$ and used for the detection of copper ions in aqueous environments (Figure 18E). ${ }^{271}$ These LnSNCs are particularly appealing, since they lack lead in their composition and are dispersible in water. The authors observed selective and pronounced quenching of $\mathrm{Eu}^{3+}$ emission upon addition of different amounts of $\mathrm{Cu}^{2+}$ in solution, which they attributed to the appearance of non-radiative relaxation pathways related to the adsorption of copper ions on the surface of the LnSNCs. This quenching was harnessed to probe $\mathrm{Cu}^{2+}$ concentration in the range from 5 $\mathrm{nM}$ to $3 \mu \mathrm{M}$. Moving from perovskites, $\mathrm{CaS}: \mathrm{Ce}^{3+}, \mathrm{Er}^{3+} \mathrm{LnSNCs}$ transferred to water with the aid of phospholipids were recently used for detection of $\mathrm{H}_{2} \mathrm{O}_{2}$ and $\mathrm{H}_{2} \mathrm{O}_{2}$-producing disease marker, xanthine (XA) (Figure 18F). ${ }^{109}$ The authors suggested that $\mathrm{H}_{2} \mathrm{O}_{2}$ could reduce $\mathrm{Ce}^{3+}$ to $\mathrm{Ce}^{4+}$ and/or disrupt the phospholipid layer, overall resulting in a decrease of the NIR emission signal under blue LED excitation. The limit of detection for $\mathrm{H}_{2} \mathrm{O}_{2}$ was $21.1 \mathrm{nM}$. Moreover, $\mathrm{XA}$ can produce $\mathrm{H}_{2} \mathrm{O}_{2}$ through the XA/XA oxidase (XOD) enzymatic reaction. Hence a suspension of LnSNCs was prepared in a solution of XOD. Upon addition of $X A, \mathrm{H}_{2} \mathrm{O}_{2}$ was produced (along with uric acid, $U A$ ), hence leading to quenching of the emission of $\mathrm{CaS}: \mathrm{Ce}^{3+}, \mathrm{Er}^{3+}$. A specific linear response range of 0.19-1000 $\mu \mathrm{M}$ and a limit of detection of $32.0 \mathrm{nM}$ were found.

\subsection{Biomedical.}

In the biomedical context, $\mathrm{Ln}^{3+}$ emission has been harnessed for fluorescence imaging, ${ }^{302-304}$ as well as for in situ triggering of photoinitiated reactions $s^{14,305}$ or stimulation of neuronal activity. ${ }^{306,307}$ However, the use of LnSNCs featuring host-sensitized $\mathrm{Ln}^{3+}$ emission in this context is very limited. NIR-emitting LnSNCs are highly sought after for in vivo applications in the biomedical context, since their emission falls within so-called biological windows (BWs) where biological tissues are more transparent to light. ${ }^{308}$ Plentiful are the examples of $\mathrm{Ln}^{3+}$-doped nanoparticles excitable in the first biological window (BW-I, 750-950 nm) and whose emission matches the second biological window (BW-II, 1000-1350 nm). ${ }^{43,309-311}$ This combination ensures good tissue penetration of the electromagnetic radiation both emitted and used for excitation. However, we could not find any report on a LnSNC which exhibits these optical characteristics. Strictly speaking, there are studies on colloidal ZnO: $\mathrm{Nd}^{3+312}$ and $\ln _{2} \mathrm{O}_{3}: \mathrm{Er}^{3+}, 115$ for instance, where those conditions are respected. However, therein NIR emission is achieved via direct excitation of the $\mathrm{Ln}^{3+}$ ion and, even when host-to- $\mathrm{Ln}^{3+} \mathrm{ET}$ is reported, the absorption of the host falls well above BWI. Yet, for in vitro fluorescence imaging there are fewer optical restrictions. To that end, Gao et al. employed $\mathrm{CaS}_{\mathrm{Eu}}{ }^{2+}, \mathrm{Sm}^{3+}$ modified with biotinylated phospholipids to target cancer cells (Colo-26, M109, HeLa) and image them under $980-\mathrm{nm}$ or $1200-\mathrm{nm}$ excitation. ${ }^{126}$ Visible emission was prompted by NIR excitation owing to the presence of electron trapped in interbandgap states (see Section 6.4). Similarly, 
Wang et al. reported on the use of a library of LnSNCs based on AESs for HepG2 cells imaging under NIR excitation. ${ }^{106}$ The authors also tested the applicability of these particles in vivo by recording fluorescence images of subcutaneously injected particles in mice. The LnSNCs featured on the surface mercaptoundecanoic acid (MUA), which is negatively charged. Modification of the surface with diamino polyethylene glycol and then with D-Mannosamine made blue-emitting SrS: $\mathrm{Ce}^{3+}, \mathrm{Sm}^{3+}, \mathrm{Mn}^{2+} \mathrm{LnSNCs}$ capable of targeting lungs. Red-emitting, MUA-bearing $\mathrm{CaS}: \mathrm{Eu}^{2+}, \mathrm{Sm}^{3+}, \mathrm{Mn}^{2+} \mathrm{LnSNCs}$ accumulated selectively at the liver. After intravenous injection of a mixture of the two particles, liver and organs were distinguishable due to the different luminescence color under NIR-excitation. The same group later showcased the use of these AES-based LnSNCs for selective in vitro and in vivo imaging of bacteria (Figure 18G). ${ }^{279}$ Teicoplanin (TEC) and polymyxin B (PMB) were used to modify the surface of CaS- and SrS-based LnSNCs, so to endow these particles with targeting capability towards strains of Gram-positive or Gram-negative bacteria, respectively. This selectivity was tested incubating the LnSNCs with bacteria cells. Even more interestingly, mice were infected via subcutaneous injection of Gram-positive S. aureus and Gram-negative E. coli. After intravenous injection of LnSNCs suspensions, the authors observed accumulation of the contrast agents at the infected lesion sites.

\section{Perspective and outlook}

Colloidal lanthanide-doped semiconductor nanocrystals are a class of materials with tremendous untapped potential. Although a deeper understanding of the subject has been achieved in terms of material design and synthesis strategies, there is still plenty of room for discoveries. We believe that the impact that these systems are poised to have in addressing societally relevant challenges in the fields of nanomedicine and renewable energy will spur more studies on LnSNCs in the next few years. However, the success is contingent on a well-planned effort.

The study of these materials is spurred on by the possibility of obtaining colloidal nanocrystals with an unparalleled bright lanthanide emission, guaranteed by strong light absorption of the host material and channeling of the optical energy to the emitting dopant ions. The resulting lanthanide emission retains its long-lived and spectrally narrow nature. This combination makes LnSNCs superior materials both in application fields where either quantum dots or state-of-the-art lanthanide-doped nanoparticles are currently the staple. More than two decades worth of investigation around methods for preparing doped colloidal SNCs have supplied strategies for the synthesis of these systems. Some successes were obtained in the past few years, but challenges still lay ahead in terms of improving the performance of the LnSNCs on several levels.

Since the cornerstones of material design are now understood, the portfolio of available LnSNCs should be enlarged. This can be accomplished via a systematic scrutiny of possible combinations of lanthanide ions and semiconductor materials, evaluating on paper if the conditions for effective doping and sensitization of $\mathrm{Ln}^{3+}$ luminescence are met. The wealth of information available on bulk $\mathrm{Ln}^{3+}$-doped semiconductors can provide solid foundation to that end. Regarding host semiconductor materials, we foresee that multinary and rare-earth-based semiconductors (along with rare-earth-based multinary systems, for that matter) will represent ideal candidates. The use of multinary semiconductors containing metals with different chemistry is particularly appealing, since it would enable leveraging selective cation exchange strategies, thus opening the door to the preparation of a plethora of otherwise inaccessible nanomaterials.

Another important step is to gain additional knowledge around the energy transfer phenomena occurring between the host material and the lanthanide ion, with a keen eye on the role played by different interbandgap states. In this vein, low-temperature spectroscopic characterization methods are pivotal. Moreover, single-particle studies have proven exceptionally useful in the investigation of property fluctuations in nanocrystals ensembles. ${ }^{262,313-315}$ The employment of this characterization 
modality is expected to afford unprecedented insight into the photophysical mechanisms. The experimental effort should also be supported by theoretical models, which will help better interpreting the behavior of the LnSNCs as well as suggest further strategies to enhance the brightness of the system.

Although in terms of optical properties the main shortcoming of $\mathrm{Ln}^{3+}$ ions is their poor absorption, boosting this parameter is not the only challenge to overcome in order to obtain brightly emitting LnSNCs. Towards that end, it is pivotal to avoid quenching phenomena and promote a host-tolanthanide transfer process with efficiency close to unity. Particularly appealing is the use of core/shell architectures in terms of reducing the interaction with solvent molecules (which possess high-energy vibrations) as well limiting non-radiative recombination occurring at surface trap states. The capability of fine tuning the relative position of the donor state (by means of size and composition control, or intentional introduction of defect-related interbandgap states) is of paramount importance to promote effective host sensitization. It is not far-fetched to think that artificial intelligence might come into play in the quest for this type of materials, ${ }^{316}$ providing the researchers with a powerful tool to predict structures with the highest likelihood to display strong host-sensitized $\mathrm{Ln}^{3+}$ luminescence.

Ultimately, the advancements suggested above are functional to the development of LnSNCs with properties tailored to the needs of different fields. As amply discussed in the previous section, the use of all-inorganic halide perovskites doped with $\mathrm{Yb}^{3+}$ is envisaged in the next generation of photovoltaic devices. Yet, halide perovskites suffer from poor stability particularly against moisture, and the most efficient systems are based on lead - an aspect which poses major concerns in the handling and, even more so, disposal of the materials at the end of their life cycle. The efforts put in the search for $\mathrm{Ln}^{3+}$ doped lead-free halide perovskites that are equally performing and more stable are thus well placed. However, the emission intensity of these materials is far from the values of CsPbX ${ }_{3}$ LnSNCs and they often feature wider bandgaps. The difficulty in pushing the absorption of perovskite nanocrystals towards longer wavelength approaching NIR is another limit of currently available materials. In that vein, a recent work from the group of Manna showcased extended absorption of lead chalcohalides, opening new opportunity also for $\mathrm{LnSNCs}^{317}$ But, again, they contain $\mathrm{Pb}^{2+}$. Thus, the development of efficiently emitting heavy metal-free LnSNCs remains one of the most important challenges.

On a different note, brightly emitting LnSNCs could find applications as efficient phosphors in lighting, while fully NIR-operating systems will be incredibly useful in the biomedical field. The development of LnSNCs that are capable to absorb and emit photons with unprecedented efficacy in one or more of the biological windows ${ }^{308}$ would represent a major breakthrough for fluorescence imaging and in vivo luminescence thermometry. Hence, LnSNCs based on lanthanide ions such as $\mathrm{Nd}^{3+}$, $\mathrm{Er}^{3+}, \mathrm{Ho}^{3+}$ and $\mathrm{Tm}^{3+}$ could constitute the next generation of optical probes to perform all-optical (hence minimally invasive) diagnosis of several health conditions with unmatched readout speed and penetration depth. Importantly, the intrinsic magnetic properties of $\mathrm{Ln}^{3+}$ offer a further imaging opportunity via magnetic resonance. Needless to say, the lack of heavy metal in the composition of LnSNCs used in this context is an even more urgent matter.

The amalgamation of optical and magnetic features in the same nanostructure is alluring also considering the development of future spintronics and opto-magnetic devices., ${ }^{7,206}$ In those devices, the magnetic properties of the LnSNC would represent the "core" feature, whereas light (absorbed and emitted) can act as an accurate means to solve addressability issues that are inherent at the nanoscale.

Finally, we also foresee exciting scenarios in the development of LnSNCs with bright emission in the MIR region ${ }^{190}$ - an electromagnetic radiation domain that has received little attention to date as far as nanocrystals are concerned, mainly because of the lack of photon detectors efficiently working in that range. However, research in this direction could pave the way for MIR nano-lasers as well as biomedical applications beyond the three NIR transparency windows. 
The possibilities are innumerable and the path to them is disseminated with several rewarding challenges. We are optimistic that the strategies outlined in this Review will provide the tools for a more rational design of lanthanide-doped colloidal semiconductor nanocrystals, their synthesis and the tailoring of their properties to the needs of technological fields such as biomedicine and photovoltaics among others.

\section{Acknowledgments}

R.M. is grateful to the European Commission for the European Union's Horizon 2020 research and innovation program, Marie Skłodowska-Curie Grant agreement No. 797945 "LANTERNS". This work was partially supported by the Ministerio de Economía y Competitividad de España (MAT2016-75362-C3-1R), by the Ministerio de Innovación y Ciencia (PID2019-106211RB-100) by the Instituto de Salud Carlos III (PI16/00812), by the Comunidad Autónoma de Madrid (B2017/BMD-3867RENIMCM), and co-financed by the European Structural and investment fund. Additional funding was provided by the European Commission Horizon 2020 project NanoTBTech. The authors would like to thank Mr. A. Skripka for his critical reading of the manuscript and suggestion to improve it. R.M. would like to thank Prof. M. Murugesu for setting an example and pushing him to pursue higher goals, as well as Prof. F. Vetrone for the encouragements and help throughout his career. Lastly, R.M. would like to thank D.J. for the constant support inside and outside the lab: your work ethic is inspirational.

\section{References}

(1) Bünzli, J.-C. G., Rising Stars in Science and Technology: Luminescent Lanthanide Materials. Eur. J. Inorg. Chem. 2017, 2017, 5058-5063.

(2) Pimentel, G. C.; Spratley, R. D., Understanding Chemistry. Holde-Day Inc., San Francisco: 1971.

(3) Bünzli, J.-C. G., On the Design of Highly Luminescent Lanthanide Complexes. Coord. Chem. Rev. 2015, 293-294, 19-47.

(4) Woodruff, D. N.; Winpenny, R. E.; Layfield, R. A., Lanthanide Single-Molecule Magnets. Chem. Rev. 2013, 113, 5110-5148.

(5) Wen, S.; Zhou, J.; Zheng, K.; Bednarkiewicz, A.; Liu, X.; Jin, D., Advances in Highly Doped Upconversion Nanoparticles. Nat. Commun. 2018, 9, 2415.

(6) Chen, G.; Qiu, H.; Prasad, P. N.; Chen, X., Upconversion Nanoparticles: Design, Nanochemistry, and Applications in Theranostics. Chem. Rev. 2014, 114, 5161-5214.

(7) Marin, R.; Brunet, G.; Murugesu, M., Shining New Light on Multifunctional Lanthanide SingleMolecule Magnets. Angew. Chem. Int. Ed. Engl. 2019, DOl: 10.1002/anie.201910299.

(8) Feynman, R. P., There's Plenty of Room at the Bottom. Engineering and Science 1960, 23, 22-36.

(9) Vetrone, F.; Naccache, R.; Zamarron, A.; Juarranz de la Fuente, A.; Sanz-Rodriguez, F.; Martinez Maestro, L.; Martin Rodriguez, E.; Jaque, D.; Garcia Sole, J.; Capobianco, J. A., Temperature Sensing Using Fluorescent Nanothermometers. ACS Nano 2010, 4, 3254-3258.

(10) Chen, C.; Wang, F.; Wen, S.; Su, Q. P.; Wu, M. C. L.; Liu, Y.; Wang, B.; Li, D.; Shan, X.; Kianinia, M., et al., Multi-Photon Near-Infrared Emission Saturation Nanoscopy Using Upconversion Nanoparticles. Nat. Commun. 2018, 9, 3290.

(11) Brites, C. D.; Xie, X.; Debasu, M. L.; Qin, X.; Chen, R.; Huang, W.; Rocha, J.; Liu, X.; Carlos, L. D., Instantaneous Ballistic Velocity of Suspended Brownian Nanocrystals Measured by Upconversion Nanothermometry. Nat. Nanotechnol. 2016, 11, 851-856.

(12) Ma, Q.; Wang, J.; Li, Z.; Lv, X.; Liang, L.; Yuan, Q., Recent Progress in Time-Resolved Biosensing and Bioimaging Based on Lanthanide-Doped Nanoparticles. Small 2019, 15, e1804969. 
(13) Dong, H.; Du, S. R.; Zheng, X. Y.; Lyu, G. M.; Sun, L. D.; Li, L. D.; Zhang, P. Z.; Zhang, C.; Yan, C. H., Lanthanide Nanoparticles: From Design toward Bioimaging and Therapy. Chem. Rev. 2015, 115, 10725-10815.

(14) Skripka, A.; Karabanovas, V.; Jarockyte, G.; Marin, R.; Tam, V.; Cerruti, M.; Rotomskis, R.; Vetrone, F., Decoupling Theranostics with Rare Earth Doped Nanoparticles. Adv. Funct. Mater. 2019, 29, 1807105.

(15) van der Ende, B. M.; Aarts, L.; Meijerink, A., Lanthanide lons as Spectral Converters for Solar Cells. Phys. Chem. Chem. Phys. 2009, 11, 11081-11095.

(16) Fischer, S.; Ivaturi, A.; Jakob, P.; Krämer, K. W.; Martin-Rodriguez, R.; Meijerink, A.; Richards, B.; Goldschmidt, J. C., Upconversion Solar Cell Measurements under Real Sunlight. Opt. Mater. 2018, 84, 389-395.

(17) Park, W.; Lu, D.; Ahn, S., Plasmon Enhancement of Luminescence Upconversion. Chem. Soc. Rev. 2015, 44, 2940-2962.

(18) Dong, J.; Gao, W.; Han, Q.; Wang, Y.; Qi, J.; Yan, X.; Sun, M., Plasmon-Enhanced Upconversion Photoluminescence: Mechanism and Application. Rev. Phys. 2019, 4, 100026.

(19) Wang, X.; Valiev, R. R.; Ohulchanskyy, T. Y.; Agren, H.; Yang, C.; Chen, G., Dye-Sensitized Lanthanide-Doped Upconversion Nanoparticles. Chem. Soc. Rev. 2017, 46, 4150-4167.

(20) Wang, J.; Deng, R., Energy Transfer in Dye-Coupled Lanthanide-Doped Nanoparticles: From Design to Application. Chem. Asian J. 2018, 13, 614-625.

(21) Yu, T.; Wei, D. M.; Li, Z.; Pan, L. J.; Zhang, Z. L.; Tian, Z. Q.; Liu, Z., Target-Modulated Sensitization of Upconversion Luminescence by Nir-Emissive Quantum Dots: A New Strategy to Construct Upconversion Biosensors. Chem. Commun. (Camb.) 2020, 56, 1976-1979.

(22) Zhang, W.; Chen, T.; Su, L.; Ge, X.; Chen, X.; Song, J.; Yang, H., Quantum Dot-Based Sensitization System for Boosted Photon Absorption and Enhanced Second near-Infrared Luminescence of Lanthanide-Doped Nanoparticle. Anal. Chem. 2020, 92, 6094-6102.

(23) Vetrone, F.; Naccache, R.; Mahalingam, V.; Morgan, C. G.; Capobianco, J. A., The ActiveCore/Active-Shell Approach: A Strategy to Enhance the Upconversion Luminescence in Lanthanide-Doped Nanoparticles. Adv. Funct. Mater. 2009, 19, 2924-2929.

(24) Zhou, B.; Tao, L.; Tsang, Y. H.; Jin, W., Core-Shell Nanoarchitecture: A Strategy to Significantly Enhance White-Light Upconversion of Lanthanide-Doped Nanoparticles. J. Mater. Chem. C 2013, 1, 4313-4318.

(25) Kang, F.; He, J.; Sun, T.; Bao, Z. Y.; Wang, F.; Lei, D. Y., Plasmonic Dual-Enhancement and Precise Color Tuning of Gold Nanorod@SiO 2 Coupled Core-Shell-Shell Upconversion Nanocrystals. Adv. Funct. Mater. 2017, 27, 1701842.

(26) Das, A.; Mao, C.; Cho, S.; Kim, K.; Park, W., Over 1000-Fold Enhancement of Upconversion Luminescence Using Water-Dispersible Metal-Insulator-Metal Nanostructures. Nat. Commun. 2018, 9, 4828.

(27) Garfield, D. J.; Borys, N. J.; Hamed, S. M.; Torquato, N. A.; Tajon, C. A.; Tian, B.; Shevitski, B.; Barnard, E. S.; Suh, Y. D.; Aloni, S., et al., Enrichment of Molecular Antenna Triplets Amplifies Upconverting Nanoparticle Emission. Nat. Photonics 2018, 12, $402-407$.

(28) Bhuckory, S.; Hemmer, E.; Wu, Y.-T.; Yahia-Ammar, A.; Vetrone, F.; Hildebrandt, N., Core or Shell? $\mathrm{Er}^{3+}$ FRET Donors in Upconversion Nanoparticles. Eur. J. Inorg. Chem. 2017, 2017, 5186-5195.

(29) Back, M.; Trave, E.; Marin, R.; Mazzucco, N.; Cristofori, D.; Riello, P., Energy Transfer in Bi- and ErCodoped $\mathrm{Y}_{2} \mathrm{O}_{3}$ nanocrystals: An Effective System for Rare Earth Fluorescence Enhancement. J. Phys. Chem. C 2014, 118, 30071-30078.

(30) Dhananjaya, N.; Nagabhushana, H.; Nagabhushana, B. M.; Sharma, S. C.; Rudraswamy, B.; Suriyamurthy, N.; Shivakumara, C.; Chakradhar, R. P. S., Synthesis, Characterization, Thermo- and Photoluminescence Properties of $\mathrm{Bi}^{3+} \mathrm{Co}-D o p e d \mathrm{Gd}_{2} \mathrm{O}_{3}: \mathrm{Eu}^{3+}$ Nanophosphors. Appl. Phys. B 2012, 107, 503-511.

(31) Ding, Y.; Guo, N.; Zhu, M.; Lv, W.; Ouyang, R.; Miao, Y.; Shao, B., Luminescence and Temperature Sensing Abilities of Zincate Phosphors Co-Doped Bismuth $\mathrm{Bi}^{3+}$ and Lanthanide $\mathrm{Eu}^{3+} / \mathrm{Sm}^{3+}$. Mater. Res. Bull. 2020, 129, 110869. 
(32) R Bajgiran, K.; Darapaneni, P.; Melvin, A. T.; Dorman, J. A., Effects of Weak Electric Field on the Photoluminescence Behavior of $\mathrm{Bi}^{3+}$-Doped $\mathrm{YVO}_{4}: \mathrm{Eu}^{3+}$ Core-Shell Nanoparticles. J. Phys. Chem. C 2019, 123, 13027-13035.

(33) Marciniak, L.; Bednarkiewicz, A.; Drabik, J.; Trejgis, K.; Strek, W., Optimization of Highly Sensitive Yag: $\mathrm{Cr}^{3+}, \mathrm{Nd}^{3+}$ Nanocrystal-Based Luminescent Thermometer Operating in an Optical Window of Biological Tissues. Phys. Chem. Chem. Phys. 2017, 19, 7343-7351.

(34) Tawalare, P. K.; Bhatkar, V. B.; Omanwar, S. K.; Moharil, S. V., $\mathrm{Cr}^{3+}$ Sensitized near Infrared Emission in $\mathrm{Al}_{2} \mathrm{O}_{3}: \mathrm{Cr}, \mathrm{Nd} / \mathrm{Yb}$ Phosphors. J. Alloys Compd. 2019, 790, 1192-1200.

(35) Marciniak, L.; Bednarkiewicz, A., Nanocrystalline NIR-to-NIR Luminescent Thermometer Based on $\mathrm{Cr}^{3+}, \mathrm{Yb}^{3+}$ Emission. Sens. Actuators B Chem. 2017, 243, 388-393.

(36) Trejgis, K.; Marciniak, L., The Influence of Manganese Concentration on the Sensitivity of Bandshape and Lifetime Luminescent Thermometers Based on $\mathrm{Y}_{3} \mathrm{Al}_{5} \mathrm{O}_{12}: \mathrm{Mn}^{3+}, \mathrm{Mn}^{4+}, \mathrm{Nd}^{3+}$ Nanocrystals. Phys. Chem. Chem. Phys. 2018, 20, 9574-9581.

(37) Peng, D.; Ju, Q.; Chen, X.; Ma, R.; Chen, B.; Bai, G.; Hao, J.; Qiao, X.; Fan, X.; Wang, F., LanthanideDoped Energy Cascade Nanoparticles: Full Spectrum Emission by Single Wavelength Excitation. Chem. Mater. 2015, 27, 3115-3120.

(38) Marin, R.; Sponchia, G.; Riello, P.; Sulcis, R.; Enrichi, F., Photoluminescence Properties of YAG:Ce ${ }^{3+}, \mathrm{Pr}^{3+}$ Phosphors Synthesized Via the Pechini Method for White Leds. J. Nanopart. Res. 2012, 14, 886.

(39) Marin, R.; Back, M.; Mazzucco, N.; Enrichi, F.; Frattini, R.; Benedetti, A.; Riello, P., Unexpected Optical Activity of Cerium in $\mathrm{Y}_{2} \mathrm{O}_{3}: \mathrm{Ce}^{3+}, \mathrm{Yb}^{3+}, \mathrm{Er}^{3+} \mathrm{Up}$ and Down-Conversion System. Dalton Trans. 2013, 42, 16837-16845.

(40) Liu, X.; Ji, Q.; Hu, Q.; Li, C.; Chen, M.; Sun, J.; Wang, Y.; Sun, Q.; Geng, B., Dual-Mode Long-Lived Luminescence of $\mathrm{Mn}^{2+}$-Doped Nanoparticles for Multilevel Anticounterfeiting. ACS Appl. Mater. Interfaces 2019, 11, 30146-30153.

(41) Adusumalli, V. N. K. B.; Koppisetti, H. V. S. R. M.; Mahalingam, V., Ce ${ }^{3+}$ Sensitized Bright White Light Emission from Colloidal $\mathrm{Ln}^{3+}$ Doped $\mathrm{CaF}_{2}$ Nanocrystals for the Development of Transparent Nanocomposites. J. Mater. Chem. C 2016, 4, 2289-2294.

(42) Fan, Y.; Liu, L.; Zhang, F., Exploiting Lanthanide-Doped Upconversion Nanoparticles with Core/Shell Structures. Nano Today 2019, 25, 68-84.

(43) Li, H.; Wang, X.; Li, X.; Zeng, S.; Chen, G., Clearable Shortwave-Infrared-Emitting NaErF 4 Nanoparticles for Noninvasive Dynamic Vascular Imaging. Chem. Mater. 2020, 32, 3365-3375.

(44) Fischer, S.; Bronstein, N. D.; Swabeck, J. K.; Chan, E. M.; Alivisatos, A. P., Precise Tuning of Surface Quenching for Luminescence Enhancement in Core-Shell Lanthanide-Doped Nanocrystals. Nano Lett. 2016, 16, 7241-7247.

(45) Zheng, W.; Zhou, S.; Chen, Z.; Hu, P.; Liu, Y.; Tu, D.; Zhu, H.; Li, R.; Huang, M.; Chen, X., Sub-10 $\mathrm{nm}$ Lanthanide-Doped $\mathrm{CaF}_{2}$ nanoprobes for Time-Resolved Luminescent Biodetection. Angew. Chem. Intl. Ed. Engl. 2013, 125, 6803-6808.

(46) Buissette, V.; Moreau, M.; Gacoin, T.; Boilot, J.-P.; Chane-Ching, J.-Y.; Le Mercier, T., Colloidal Synthesis of Luminescent Rhabdophane $\mathrm{LaPO}_{4}: \mathrm{Ln}^{3+} \cdot \mathrm{xH}_{2} \mathrm{O}(\mathrm{Ln}=\mathrm{Ce}, \mathrm{Tb}, \mathrm{Eu} ; \mathrm{x} \approx 0.7)$ Nanocrystals. Chem. Mater. 2004, 16, 3767-3773.

(47) Yu, M.; Zhao, Z., Plasmon-Enhanced Up-conversion Luminescence and Oxygen Vacancy DefectInduced Yellow Light in Annealed $\mathrm{Cu}_{8} \mathrm{~S}_{5} @ \mathrm{SiO}_{2} @ \mathrm{Er}_{2} \mathrm{O}_{3}$ Nanocomposites. J. Lumin. 2020, 225, 117361.

(48) Swabeck, J. K.; Fischer, S.; Bronstein, N. D.; Alivisatos, A. P., Broadband Sensitization of Lanthanide Emission with Indium Phosphide Quantum Dots for Visible to near-Infrared Downshifting. J. Am. Chem. Soc. 2018, 140, 9120-9126.

(49) Martin-Rodriguez, R.; Geitenbeek, R.; Meijerink, A., Incorporation and Luminescence of $\mathrm{Yb}^{3+}$ in CdSe Nanocrystals. J. Am. Chem. Soc. 2013, 135, 13668-13671.

(50) Creutz, S. E.; Fainblat, R.; Kim, Y.; De Siena, M. C.; Gamelin, D. R., A Selective Cation Exchange Strategy for the Synthesis of Colloidal $\mathrm{Yb}^{3+}$-Doped Chalcogenide Nanocrystals with Strong 
Broadband Visible Absorption and Long-Lived near-Infrared Emission. J. Am. Chem. Soc. 2017, 139, 11814-11824.

(51) Pan, G.; Bai, X.; Yang, D.; Chen, X.; Jing, P.; Qu, S.; Zhang, L.; Zhou, D.; Zhu, J.; Xu, W., et al., Doping Lanthanide into Perovskite Nanocrystals: Highly Improved and Expanded Optical Properties. Nano Lett. 2017, 17, 8005-8011.

(52) Zhou, D.; Sun, R.; Xu, W.; Ding, N.; Li, D.; Chen, X.; Pan, G.; Bai, X.; Song, H., Impact of Host Composition, Codoping, or Tridoping on Quantum-Cutting Emission of Ytterbium in Halide Perovskite Quantum Dots and Solar Cell Applications. Nano Lett. 2019, 19, 6904-6913.

(53) Chengelis, D. A.; Yingling, A. M.; Badger, P. D.; Shade, C. M.; Petoud, S., Incorporating Lanthanide Cations with Cadmium Selenide Nanocrystals: A Strategy to Sensitize and Protect Tb(III). J. Am. Chem. Soc. 2005, 127, 16752-16753.

(54) Mukherjee, P.; Shade, C. M.; Yingling, A. M.; Lamont, D. N.; Waldeck, D. H.; Petoud, S., Lanthanide Sensitization in li-Vi Semiconductor Materials: A Case Study with Terbium(III) and Europium(III) in Zinc Sulfide Nanoparticles. J. Phys. Chem. A 2011, 115, 4031-4041.

(55) Mir, W. J.; Sheikh, T.; Arfin, H.; Xia, Z.; Nag, A., Lanthanide Doping in Metal Halide Perovskite Nanocrystals: Spectral Shifting, Quantum Cutting and Optoelectronic Applications. NPG Asia Mater. 2020, 12, 9.

(56) Liu, Y.; Rong, X.; Li, M.; Molokeev, M. S.; Zhao, J.; Xia, Z., Incorporating Rare-Earth Terbium(III) lons into $\mathrm{Cs}_{2} \mathrm{Ag}_{\mathrm{InCl}}$ :Bi Nanocrystals toward Tunable Photoluminescence. Angew. Chem. Int. Ed. Engl. 2020, 59, 11634-11640.

(57) Bünzli, J. C.; Piguet, C., Taking Advantage of Luminescent Lanthanide Ions. Chem. Soc. Rev. 2005, 34, 1048-1077.

(58) Armelao, L.; Quici, S.; Barigelletti, F.; Accorsi, G.; Bottaro, G.; Cavazzini, M.; Tondello, E., Design of Luminescent Lanthanide Complexes: From Molecules to Highly Efficient Photo-Emitting Materials. Coord. Chem. Rev. 2010, 254, 487-505.

(59) Mickey, C. D., Some Aspects of Coordination Chemistry. J. Chem. Educ. 1981, $58,257$.

(60) Raubach, C. W.; Gouveia, A. F.; de Santana, Y. V. B.; Varela, J. A.; Ferrer, M. M.; Li, M. S.; Longo, E., Towards Controlled Synthesis and Better Understanding of Blue Shift of the CaS Crystals. J. Mater. Chem. C 2014, 2, 2743-2750.

(61) Pandey, R.; Sivaraman, S., Spectroscopic Properties of Defects in Alkaline-Earth Sulfides. J. Phys. Chem. Solids 1991, 52, 211-225.

(62) Meulenberg, R. W.; Lee, J. R.; Wolcott, A.; Zhang, J. Z.; Terminello, L. J.; van Buuren, T., Determination of the Exciton Binding Energy in CdSe Quantum Dots. ACS Nano 2009, 3, 325-330.

(63) Smith, A. M.; Nie, S., Semiconductor Nanocrystals: Structure, Properties, and Band Gap Engineering. Acc. Chem. Res. 2010, 43, 190-200.

(64) Pelant, I.; Valenta, J., Luminescence of Excitons. In Luminescence Spectroscopy of Semiconductors. Oxford Scholarship Online, 2012; pp 161-204.

(65) de Mello Donegá, C., Nanoparticles: Workhorses or Nanoscience. Springer-Verlag Berlin Heidelberg, 2014.

(66) Brus, L., Electronic Wave Functions in Semiconductor Clusters: Experiment and Theory. J. Phys. Chem. 1986, 90, 2555-2560.

(67) Shen, Y.; Lifante, J.; Ximendes, E.; Santos, H. D. A.; Ruiz, D.; Juarez, B. H.; Zabala Gutierrez, I.; Torres Vera, V.; Rubio Retama, J.; Martin Rodriguez, E., et al., Perspectives for $\mathrm{Ag}_{2} \mathrm{~S}$ NIR-II Nanoparticles in Biomedicine: From Imaging to Multifunctionality. Nanoscale 2019, 11, 19251-19264.

(68) Giansante, C.; Infante, I., Surface Traps in Colloidal Quantum Dots: A Combined Experimental and Theoretical Perspective. J. Phys. Chem. Lett. 2017, 8, 5209-5215.

(69) Bailey, R. E.; Nie, S., Alloyed Semiconductor Quantum Dots: Tuning the Optical Properties without Changing the Particle Size. J. Am. Chem. Soc. 2003, 125, 7100-7106.

(70) Akkerman, Q. A.; Genovese, A.; George, C.; Prato, M.; Moreels, I.; Casu, A.; Marras, S.; Curcio, A.; Scarpellini, A.; Pellegrino, T., et al., From Binary $\mathrm{Cu}_{2} \mathrm{~S}$ to Ternary Cu-In-S and Quaternary Cu-In-Zn-S Nanocrystals with Tunable Composition Via Partial Cation Exchange. ACS Nano 2015, 9, 521-531. 
(71) Zhang, W.; Zhong, X., Facile Synthesis of ZnS-CulnS 2 -Alloyed Nanocrystals for a Color-Tunable Fluorchrome and Photocatalyst. Inorg. Chem. 2011, 50, 4065-4072.

(72) Nedelcu, G.; Protesescu, L.; Yakunin, S.; Bodnarchuk, M. I.; Grotevent, M. J.; Kovalenko, M. V., Fast Anion-Exchange in Highly Luminescent Nanocrystals of Cesium Lead Halide Perovskites (CsPbX ${ }_{3}$ $\mathrm{X}=\mathrm{Cl}, \mathrm{Br}, \mathrm{I})$. Nano Lett. 2015, 15, 5635-5640.

(73) Vegard, L., Die Konstitution Der Mischkristalle Und Die Raumfllung Der Atome. Z. Phys. 1921, 5, 1726.

(74) Luo, Y.; Hao, S.; Cai, S.; Slade, T. J.; Luo, Z. Z.; Dravid, V. P.; Wolverton, C.; Yan, Q.; Kanatzidis, M. G., High Thermoelectric Performance in the New Cubic Semiconductor AgSnSbSe 3 by High-Entropy Engineering. J. Am. Chem. Soc. 2020, 142, 15187-15198.

(75) Zeman, O. E. O.; von Rohr, F. O.; Neudert, L.; Schnick, W., Facile One-Step Synthesis of $Z n_{1-x} M n_{x} S_{i N}$ Nitride Semiconductor Solid Solutions Via Solid-State Metathesis Reaction. Z. Anorg. Allg. Chem. 2020, 646, 228-233.

(76) Liou, B. T.; Yen, S. H.; Kuo, Y. K., Vegard's Law Deviation in Band Gap and Bowing Parameter of Al $\left.\right|_{x} \operatorname{nn}_{1-x} N$. Appl. Phys. A 2005, 81, 651-655.

(77) Mocatta, D.; Cohen, G.; Schattner, J.; Millo, O.; Rabani, E.; Banin, U., Heavily Doped Semiconductor Nanocrystal Quantum Dots. Science 2011, 332, 77-81.

(78) Fainblat, R.; Barrows, C. J.; Hopmann, E.; Siebeneicher, S.; Vlaskin, V. A.; Gamelin, D. R.; Bacher, G., Giant Excitonic Exchange Splittings at Zero Field in Single Colloidal CdSe Quantum Dots Doped with Individual $\mathrm{Mn}^{2+}$ Impurities. Nano Lett. 2016, 16, 6371-6377.

(79) Cotton, S. A.; Harrowfield, J. M., Lanthanides: Coordination Chemistry. In Encyclopedia of Inorganic and Bioinorganic Chemistry, Wiley-VCH, Hoboken, New Jersey, 2012.

(80) Jain, A.; Ong, S. P.; Hautier, G.; Chen, W.; Richards, W. D.; Dacek, S.; Cholia, S.; Gunter, D.; Skinner, D.; Ceder, G., et al., Commentary: The Materials Project: A Materials Genome Approach to Accelerating Materials Innovation. APL Mater. 2013, 1, 011002.

(81) http://abulafia.mt.ic.ac.uk/shannon/.

(82) Shannon, R. D., Revised Effective Ionic Radii and Systematic Studies of Interatomic Distances in Halides and Chalcogenides. Acta Crystallogr. A 1976, 32, 751-767.

(83) Mukherjee, S.; Sudarsan, V.; Vatsa, R. K.; Tyagi, A. K., Luminescence Studies on Lanthanide Ions $\left(\mathrm{Eu}^{3+}, \mathrm{Dy}^{3+}\right.$ and $\mathrm{Tb}^{3+}$ Doped YAG:Ce Nano-Phosphors. J. Lumin. 2009, 129, 69-72.

(84) Milstein, T. J.; Kroupa, D. M.; Gamelin, D. R., Picosecond Quantum Cutting Generates Photoluminescence Quantum Yields over 100\% in Ytterbium-Doped CsPbCl ${ }_{3}$ Nanocrystals. Nano Lett. 2018, 18, 3792-3799.

(85) Groot-Berning, K.; Kornher, T.; Jacob, G.; Stopp, F.; Dawkins, S. T.; Kolesov, R.; Wrachtrup, J.; Singer, K.; Schmidt-Kaler, F., Deterministic Single-Ion Implantation of Rare-Earth lons for NanometerResolution Color-Center Generation. Phys. Rev. Lett. 2019, 123, 106802.

(86) Ruterana, P.; Chauvat, M.-P.; Lorenz, K., Mechanisms of Damage Formation During Rare Earth lon Implantation in Nitride Semiconductors. Jap. J. Appl. Phys. 2013, 52, $11 \mathrm{NHO2.}$

(87) Zhang, H.; Shen, Y., Luminescence of ZnS:Ho under Excitation around the Band Edge (EBE). J. Lumin. 1988, 40-41, 401-402.

(88) Bol, A. A.; van Beek, R.; Meijerink, A., On the Incorporation of Trivalent Rare Earth lons in II-VI Semiconductor Nanocrystals. Chem. Mater. 2002, 14, 1121-1126.

(89) Anderson, W. W., Luminescence of Rare-Earth-Activated Cadmium Sulfide. J. Chem. Phys. 1966, 44, 3283-3288.

(90) Radevici, l.; Sushkevich, K.; Sirkeli, V.; Huhtinen, H.; Nedeoglo, D.; Paturi, P., Luminescent Properties of the ZnSe:Yb Crystals in the Visible Spectral Range. J. Lumin. 2013, 143, 275-279.

(91) Sinha, G.; Patra, A., Generation of Green, Red and White Light from Rare-Earth Doped $\mathrm{Ga}_{2} \mathrm{O}_{3}$ Nanoparticles. Chem. Phys. Lett. 2009, 473, 151-154.

(92) Layek, A.; Yildirim, B.; Ghodsi, V.; Hutfluss, L. N.; Hegde, M.; Wang, T.; Radovanovic, P. V., Dual Europium Luminescence Centers in Colloidal $\mathrm{Ga}_{2} \mathrm{O}_{3}$ Nanocrystals: Controlled in Situ Reduction of Eu(III) and Stabilization of Eu(II). Chem. Mater. 2015, 27, 6030-6037. 
(93) Wang, T.; Layek, A.; Hosein, I. D.; Chirmanov, V.; Radovanovic, P. V., Correlation between Native Defects and Dopants in Colloidal Lanthanide-Doped $\mathrm{Ga}_{2} \mathrm{O}_{3}$ nanocrystals: A Path to Enhance Functionality and Control Optical Properties. J. Mater. Chem. C 2014, 2, 3212-3222.

(94) Zhu, H.; Li, R.; Luo, W.; Chen, X., Eu ${ }^{3+}$-Doped Beta- $\mathrm{Ga}_{2} \mathrm{O}_{3}$ Nanophosphors: Annealing Effect, Electronic Structure and Optical Spectroscopy. Phys. Chem. Chem. Phys. 2011, 13, 4411-4419.

(95) Ghosh, S.; Das, K.; Sinha, G.; Lahtinen, J.; De, S. K., Bright White Light Emitting Eu and Tb Co-Doped Monodisperse $\operatorname{In}_{2} \mathrm{O}_{3}$ Nanocrystals. J. Mater. Chem. C 2013, 1, 5557-5566.

(96) Vela, J.; Prall, B. S.; Rastogi, P.; Werder, D. J.; Casson, J. L.; Williams, D. J.; Klimov, V. I.; Hollingsworth, J. A., Sensitization and Protection of Lanthanide Ion Emission in $\ln _{2} \mathrm{O}_{3}$ :Eu Nanocrystal Quantum Dots. J. Phys. Chem. C 2008, 112, 20246-20250.

(97) Xiao, Q.; Liu, Y.; Liu, L.; Li, R.; Luo, W.; Chen, X., Eu ${ }^{3+}$-Doped $\ln _{2} \mathrm{O}_{3}$ nanophosphors: Electronic Structure and Optical Characterization. J. Phys. Chem. C 2010, 114, 9314-9321.

(98) Planelles-Aragó, J.; Cordoncillo, E.; Ferreira, R. A. S.; Carlos, L. D.; Escribano, P., Synthesis, Characterization and Optical Studies on Lanthanide-Doped CdS Quantum Dots: New Insights on $\mathrm{CdS} \rightarrow$ Lanthanide Energy Transfer Mechanisms. J. Mater. Chem. 2011, 21, 1162-1170.

(99) Saif, M.; Abdel-Mottaleb, M. S. A., Titanium Dioxide Nanomaterial Doped with Trivalent Lanthanide Ions of Tb, Eu and Sm: Preparation, Characterization and Potential Applications. Inorg. Chim. Acta 2007, 360, 2863-2874.

(100) Luo, W.; Li, R.; Chen, X., Host-Sensitized Luminescence of $\mathrm{Nd}^{3+}$ and $\mathrm{Sm}^{3+}$ Ions Incorporated in Anatase Titania Nanocrystals. J. Phys. Chem. C 2009, 113, 8772-8777.

(101) Zhang, Y.; Zhang, H.; Xu, Y.; Wang, Y., Europium Doped Nanocrystalline Titanium Dioxide: Preparation, Phase Transformation and Photocatalytic Properties. J. Mater. Chem. 2003, 13, 22612265.

(102) Shah, S. I.; Li, W.; Huang, C. P.; Jung, O.; Ni, C., Study of Nd ${ }^{3+}, \mathrm{Pd}^{2+}, \mathrm{Pt}^{4+}$, and Fe ${ }^{3+}$ Dopant Effect on Photoreactivity of $\mathrm{TiO}_{2}$ Nanoparticles. Proc. Natl. Acad. Sci. USA 2002, 99 Suppl 2, 6482-6486.

(103) Ma, J.-P.; Chen, Y.-M.; Zhang, L.-M.; Guo, S.-Q.; Liu, J.-D.; Li, H.; Ye, B.-J.; Li, Z.-Y.; Zhou, Y.; Zhang, B.-B., et al., Insights into the Local Structure of Dopants, Doping Efficiency, and Luminescence Properties of Lanthanide-Doped CsPbCl 3 Perovskite Nanocrystals. J. Mater. Chem. C 2019, 7, 30373048.

(104) Zheng, X.; Hou, Y.; Sun, H. T.; Mohammed, O. F.; Sargent, E. H.; Bakr, O. M., Reducing Defects in Halide Perovskite Nanocrystals for Light-Emitting Applications. J. Phys. Chem. Lett. 2019, 10, 26292640.

(105) Lu, C. H.; Biesold-McGee, G. V.; Liu, Y.; Kang, Z.; Lin, Z., Doping and Ion Substitution in Colloidal Metal Halide Perovskite Nanocrystals. Chem. Soc. Rev. 2020, 49, 4953-5007.

(106) Wang, J.; Zhu, Y.; Grimes, C. A.; Cai, Q., Multicolor Lanthanide-Doped CaS and SrS Near-Infrared Stimulated Luminescent Nanoparticles with Bright Emission: Application in Broad-Spectrum Lighting, Information Coding, and Bio-Imaging. Nanoscale 2019, 11, 12497-12501.

(107) Zhao, Y.; Rabouw, F. T.; van Puffelen, T.; van Walree, C. A.; Gamelin, D. R.; de Mello Donegá, C.; Meijerink, A., Lanthanide-Doped CaS and SrS Luminescent Nanocrystals: A Single-Source Precursor Approach for Doping. J. Am. Chem. Soc. 2014, 136, 16533-16543.

(108) Rodríguez Burbano, D. C.; Rodríguez, E. M.; Dorenbos, P.; Bettinelli, M.; Capobianco, J. A., The NearIR Photo-Stimulated Luminescence of $\mathrm{CaS}_{\mathrm{Eu}}{ }^{2+} / \mathrm{Dy}^{3+}$ Nanophosphors. J. Mater. Chem. C 2014, 2, 228-231.

(109) Zhang, M.; Zheng, W.; Liu, Y.; Huang, P.; Gong, Z; Wei, J.; Gao, Y.; Zhou, S.; Li, X.; Chen, X., A New Class of Blue-Led-Excitable NIR-II Luminescent Nanoprobes Based on Lanthanide-Doped CaS Nanoparticles. Angew. Chem. Int. Ed. Engl. 2019, 58, 9556-9560.

(110) Garcia, J.; Allen, M. J., Developments in the Coordination Chemistry of Europium(II). Eur. J. Inorg. Chem. 2012, 2012, 4550-4563.

(111) Alam, F.; Wegner, K. D.; Pouget, S.; Amidani, L.; Kvashnina, K.; Aldakov, D.; Reiss, P., Eu²+: A Suitable Substituent for $\mathrm{Pb}^{2+}$ in CsPbX 3 Perovskite Nanocrystals? J. Chem. Phys. 2019, 151, 231101. 
(112) Huang, J.; Lei, T.; Siron, M.; Zhang, Y.; Yu, S.; Seeler, F.; Dehestani, A.; Quan, L. N.; Schierle-Arndt, K.; Yang, P., Lead-Free Cesium Europium Halide Perovskite Nanocrystals. Nano Lett. 2020, 20, 37343739.

(113) Suta, M.; Wickleder, C., Synthesis, Spectroscopic Properties and Applications of Divalent Lanthanides Apart from Eu². J. Lumin. 2019, 210, 210-238.

(114) Wang, C.; Zhang, D.; Xu, L.; Jiang, Y.; Dong, F.; Yang, B.; Yu, K.; Lin, Q., A Simple Reducing Approach Using Amine to Give Dual Functional EuSe Nanocrystals and Morphological Tuning. Angew. Chem. Int. Ed. Engl. 2011, 50, 7587-7591.

(115) Xiao, Q.; Zhu, H.; Tu, D.; Ma, E.; Chen, X., Near-Infrared-to-near-Infrared Downshifting and NearInfrared-to-Visible Upconverting Luminescence of $\mathrm{Er}^{3+}$-Doped $\mathrm{In}_{2} \mathrm{O}_{3}$ Nanocrystals. J. Phys. Chem. C 2013, 117, 10834-10841.

(116) Elward, J. M.; Chakraborty, A., Effect of Dot Size on Exciton Binding Energy and Electron-Hole Recombination Probability in CdSe Quantum Dots. J. Chem. Theory. Comput. 2013, 9, 4351-4359.

(117) Li, X.; Duan, S.; Liu, H.; Chen, G.; Luo, Y.; Agren, H., Mechanism for the Extremely Efficient Sensitization of $\mathrm{Yb}^{3+}$ Luminescence in $\mathrm{CsPbCl}_{3}$ Nanocrystals. J. Phys. Chem. Lett. 2019, 10, 487-492.

(118) Gupta, S. K.; Abdou, M.; Zuniga, J. P.; Puretzky, A. A.; Mao, Y., Samarium-Activated $\mathrm{La}_{2} \mathrm{Hf}_{2} \mathrm{O}_{7}$ Nanoparticles as Multifunctional Phosphors. ACS Omega 2019, 4, 17956-17966.

(119) Li, S.; Luo, J.; Liu, J.; Tang, J., Self-Trapped Excitons in All-Inorganic Halide Perovskites: Fundamentals, Status, and Potential Applications. J. Phys. Chem. Lett. 2019, 10, $1999-2007$.

(120) Bogardus, E. H.; Bebb, H. B., Bound-Exciton, Free-Exciton, Band-Acceptor, Donor-Acceptor, and Auger Recombination in Gaas. Phys. Rev. 1968, 176, 993-1002.

(121) Marin, R.; Oussta, F.; Katea, S. N.; Prabhudev, S.; Botton, G. A.; Westin, G.; Hemmer, E., EuropiumDoped ZnO Nanosponges - Controlling Optical Properties and Photocatalytic Activity. J. Mater. Chem. C 2019, 7, 3909-3919.

(122) Ahmed, S. M.; Szymanski, P.; El-Nadi, L. M.; El-Sayed, M. A., Energy-Transfer Efficiency in Eu-Doped ZnO Thin Films: The Effects of Oxidative Annealing on the Dynamics and the Intermediate Defect States. ACS Appl. Mater. Interfaces 2014, 6, 1765-1772.

(123) Krustok, J.; Raudoja, J.; Collan, H., Photoluminescence and the Tetragonal Distortion in CulnS2. Thin Solid Films 2001, 387, 195-197.

(124) Fuhr, A. S.; Yun, H. J.; Makarov, N. S.; Li, H.; McDaniel, H.; Klimov, V. I., Light Emission Mechanisms in CulnS 2 Quantum Dots Evaluated by Spectral Electrochemistry. ACS Photonics 2017, 4, 24252435.

(125) Berends, A. C.; Mangnus, M. J. J.; Xia, C.; Rabouw, F. T.; de Mello Donegá, C., Optoelectronic Properties of Ternary I-III-V| $\left.\right|_{2}$ Semiconductor Nanocrystals: Bright Prospects with Elusive Origins. J. Phys. Chem. Lett. 2019, 10, 1600-1616.

(126) Gao, Y.; Li, R.; Zheng, W.; Shang, X.; Wei, J.; Zhang, M.; Xu, J.; You, W.; Chen, Z.; Chen, X., Broadband NIR Photostimulated Luminescence Nanoprobes Based on CaS:Eu ${ }^{2+}, \mathrm{Sm}^{3+}$ Nanocrystals. Chem. Sci. 2019, 10, 5452-5460.

(127) Yuan, L.-D.; Deng, H.-X.; Li, S.-S.; Wei, S.-H.; Luo, J.-W., Unified Theory of Direct or Indirect BandGap Nature of Conventional Semiconductors. Phys. Rev. B 2018, 98, 245203.

(128) Robel, I.; Gresback, R.; Kortshagen, U.; Schaller, R. D.; Klimov, V. I., Universal Size-Dependent Trend in Auger Recombination in Direct-Gap and Indirect-Gap Semiconductor Nanocrystals. Phys. Rev. Lett. 2009, 102, 177404.

(129) Delerue, C.; Allan, G.; Reynaud, C.; Guillois, O.; Ledoux, G.; Huisken, F., Multiexponential Photoluminescence Decay in Indirect-Gap Semiconductor Nanocrystals. Phys. Rev. B 2006, 73, 235318.

(130) Hafez, H.; Saif, M.; Abdel-Mottaleb, M. S. A., Down-Converting Lanthanide Doped $\mathrm{TiO}_{2}$ Photoelectrodes for Efficiency Enhancement of Dye-Sensitized Solar Cells. J. Power Sources 2011, $196,5792-5796$.

(131) Zhou, L.; Tanner, P. A.; Zhou, W.; Ai, Y.; Ning, L.; Wu, M. M.; Liang, H., Unique Spectral Overlap and Resonant Energy Transfer between Europium(II) and Ytterbium(III) Cations: No Quantum Cutting. Angew. Chem. Int. Ed. Engl. 2017, 56, 10357-10361. 
(132) Katayama, Y.; Kayumi, T.; Ueda, J.; Dorenbos, P.; Viana, B.; Tanabe, S., The Role of $L n^{3+}(L n=E u, ~ Y b)$ in Persistent Red Luminescence in $\mathrm{MgGeO}_{3}: \mathrm{Mn}^{2+}$. J. Mater. Chem. C 2017, 5, 8893-8900.

(133) Dorenbos, P., Systematic Behaviour in Trivalent Lanthanide Charge Transfer Energies. J. Phys. Cond. Matter 2003, 15, 8417-8434.

(134) Dorenbos, P., Lanthanide Charge Transfer Energies and Related Luminescence, Charge Carrier Trapping, and Redox Phenomena. J. Alloys Compd. 2009, 488, 568-573.

(135) Dorenbos, P., Locating Lanthanide Impurity Levels in the Forbidden Band of Host Crystals. J. Lumin. 2004, 108, 301-305.

(136) Jørgensen, C. K., Modern Aspects of Ligand Field Theory. North-Holland Publishing Company: Amsterdam, 1971.

(137) Allred, A. L., Electronegativity Values from Thermochemical Data. J. Inorg. Nucl. Chem. 1961, 17, 215-221.

(138) Dorenbos, P., The Charge Transfer Energy and the Relation with the Band Gap of Compounds. J. Lumin. 2005, 111, 89-104.

(139) Dorenbos, P., Ce ${ }^{3+} 5 \mathrm{~d}$-Centroid Shift and Vacuum Referred 4f-Electron Binding Energies of All Lanthanide Impurities in 150 Different Compounds. J. Lumin. 2013, 135, 93-104.

(140) Van Der Voort, D.; Blasse, G., The Luminescence of Europium(III) and Cerium(III) in Calcium Sulfate: Activators with an Effective Charge. J. Solid State Chem. 1990, 87, 350-359.

(141) Klik, M. A.; Gregorkiewicz, T.; Bradley, I. V.; Wells, J. P., Optically Induced Deexcitation of Rare-Earth Ions in a Semiconductor Matrix. Phys. Rev. Lett. 2002, 89, 227401.

(142) Dorenbos, P., Charge Transfer Bands in Optical Materials and Related Defect Level Location. Opt. Mater. 2017, 69, 8-22.

(143) Dorenbos, P., A Review on How Lanthanide Impurity Levels Change with Chemistry and Structure of Inorganic Compounds. ECS J. Solid State Sci. Technol. 2013, 2, R3001-R3011.

(144) Havlák, L.; Jarý, V.; Nikl, M.; Boháček, P.; Bárta, J., Preparation, Luminescence and Structural Properties of Re-Doped RbLaS 2 Compounds. Acta Mater. 2011, 59, 6219-6227.

(145) Gerner, P.; Güdel, H. U., Absorption and Upconversion Light Emission Properties of $\mathrm{Er}^{3+}$ and $\mathrm{Yb}^{3+} / \mathrm{Er}^{3+}$ Codoped NaYS . Chem. Phys. Lett. 2005, 413, 105-109.

(146) Bohnert, G.; Weber, J.; Scholz, F.; Hangleiter, A., Experimental Evidence for Charge-Transfer Excitation of Yb in InP. Appl. Phys. Lett. 1993, 63, 382-384.

(147) Manna, P.; Debnath, G. H.; Waldeck, D. H.; Mukherjee, P., What Is Beyond Charge Trapping in Semiconductor Nanoparticle Sensitized Dopant Photoluminescence? J. Phys. Chem. Lett. 2018, 9, 6191-6197.

(148) Qin, X.; Liu, X.; Huang, W.; Bettinelli, M.; Liu, X., Lanthanide-Activated Phosphors Based on 4f-5d Optical Transitions: Theoretical and Experimental Aspects. Chem. Rev. 2017, 117, 4488-4527.

(149) Duan, C. K.; Tanner, P. A.; Meijerink, A.; Makhov, V., 4f-5d Transitions of Tb ${ }^{3+}$ in $\mathrm{Cs}_{2} \mathrm{NaYF}_{6}$ : The Effect of Distortion of the Excited-State Configuration. J. Phys. Chem. A 2011, 115, 9188-9191.

(150) Dorenbos, P., Valence Stability of Lanthanide Ions in Inorganic Compounds. Chem. Mater. 2005, 17, 6452-6456.

(151) Qiao, J.; Zhou, G.; Zhou, Y.; Zhang, Q.; Xia, Z., Divalent Europium-Doped Near-Infrared-Emitting Phosphor for Light-Emitting Diodes. Nat. Commun. 2019, 10, 5267.

(152) Dong, L.; Zhang, L.; Jia, Y.; Shao, B.; Lü, W.; Zhao, S.; You, H., Synthesis, Luminescence and Application of Novel Europium, Cerium and Terbium-Doped Apatite Phosphors. CrystEngComm 2019, 21, 6226-6237.

(153) Zhong, J.; Zhuo, Y.; Hariyani, S.; Zhao, W.; Zhuang, W.; Brgoch, J., Thermally Robust and ColorTunable Blue-Green-Emitting BaMgSi $\mathrm{O}_{10}: \mathrm{Eu}^{2+}, \mathrm{Mn}^{2+}$ Phosphor for Warm-White Leds. Inorg. Chem. 2020, 59, 13427-13434.

(154) Terraschke, H.; Wickleder, C., UV, Blue, Green, Yellow, Red, and Small: Newest Developments on $\mathrm{Eu}^{2+}$-Doped Nanophosphors. Chem. Rev. 2015, 115, 11352-11378.

(155) Ueda, J.; Tanabe, S., Review of Luminescent Properties of $\mathrm{Ce}^{3+}$-Doped Garnet Phosphors: New Insight into the Effect of Crystal and Electronic Structure. Opt. Mater. X 2019, 1, 100018. 
(156) Carnall, W. T.; Fields, P. R.; Rajnak, K., Electronic Energy Levels in the Trivalent Lanthanide Aquo Ions. I. $\mathrm{Pr}^{3+}, \mathrm{Nd}^{3+}, \mathrm{Pm}^{3+}, \mathrm{Sm}^{3+}, \mathrm{Dy}^{3+}, \mathrm{Ho}^{3+}, \mathrm{Er}^{3+}$, and $\mathrm{Tm}^{3+}$. J. Chem. Phys. 1968, 49, 4424-4442.

(157) Cheng, H.; Huang, B.; Lu, J.; Wang, Z.; Xu, B.; Qin, X.; Zhang, X.; Dai, Y., Synergistic Effect of Crystal and Electronic Structures on the Visible-Light-Driven Photocatalytic Performances of $\mathrm{Bi}_{2} \mathrm{O}_{3}$ Polymorphs. Phys. Chem. Chem. Phys. 2010, 12, 15468-15475.

(158) Playford, H. Y.; Hannon, A. C.; Barney, E. R.; Walton, R. I., Structures of Uncharacterised Polymorphs of Gallium Oxide from Total Neutron Diffraction. Chem. Eur. J. 2013, 19, 2803-2813.

(159) Abiko, Y.; Nakayama, N.; Akimoto, K.; Yao, T., Difference in Luminescence Properties between Sm Doped Zns and Eu Doped Zns. Phys. Status Solidi B 2002, 229, 339-342.

(160) Sun, P.; Li, Y.; Meng, X.; Yu, S.; Liu, Y.; Liu, F.; Wang, Z., The Magnetic Field Effect on Optical Properties of Sm-Doped GaN Thin Films. J. Mater. Sci. Mater. Electron. 2014, 25, 2974-2978.

(161) Savchuk, A. I.; Paranchych, S. Y.; Frasunyak, V. M.; Fediv, V. I.; Tanasyuk, Y. V.; Kandyba, Y. O.; Nikitin, P. I., Optical and Magnetooptical Study of CdTe Crystals Doped with Rare Earth Ions. Mater. Sci. Eng. B 2003, 105, 161-164.

(162) Chen, L.; Zhang, J.; Lu, S.; Ren, X.; Wang, X., On the Energy Transfer from Nanocrystalline ZnS to $\mathrm{Tb}^{3+}$ Ions Confined in Reverse Micelles. Chem. Phys. Lett. 2005, 409, 144-148.

(163) Dethlefsen, J. R.; Mikhailovsky, A. A.; Burks, P. T.; Døssing, A.; Ford, P. C., Lanthanide Modification of CdSe/ZnS Core/Shell Quantum Dots. J. Phys. Chem. C 2012, 116, 23713-23720.

(164) Sun, B.; Yi, G.; Chen, D.; Zhou, Y.; Cheng, J., Synthesis and Characterization of Strongly Fluorescent Europium-Doped Calcium Sulfide Nanoparticles. J. Mater. Chem. 2002, 12, 1194-1198.

(165) Bessière, A.; Dorenbos, P.; van Eijk, C. W. E.; Yamagishi, E.; Hidaka, C.; Takizawa, T., Spectroscopy and Lanthanide Impurity Level Locations in $\mathrm{CaGa}_{2} \mathrm{~S} 4: \mathrm{Ln}^{3+}(\mathrm{Ln}=\mathrm{Ce}, \mathrm{Pr}, \mathrm{Tb}, \mathrm{Er}, \mathrm{Tm})$. J. Electrochem. Soc. 2004, 151, H254-H260.

(166) Allen, P. M.; Bawendi, M. G., Ternary I-III-VI Quantum Dots Luminescent in the Red to Near-Infrared. J. Am. Chem. Soc. 2008, 130, 9240-9241.

(167) Girma, W. M.; Fahmi, M. Z.; Permadi, A.; Abate, M. A.; Chang, J. Y., Synthetic Strategies and Biomedical Applications of I-III-VI Ternary Quantum Dots. J. Mater. Chem. B 2017, 5, 6193-6216.

(168) Kolny-Olesiak, J.; Weller, H., Synthesis and Application of Colloidal CulnS 2 Semiconductor Nanocrystals. ACS Appl. Mater. Interfaces 2013, 5, 12221-12237.

(169) Menyuk, N.; Dwight, K.; Pierce, J. W., NaYF 4 :Yb,Er—an Efficient Upconversion Phosphor. Appl. Phys. Lett. 1972, 21, 159-161.

(170) Boyer, J. C.; Vetrone, F.; Cuccia, L. A.; Capobianco, J. A., Synthesis of Colloidal Upconverting NaYF Nanocrystals Doped with $\mathrm{Er}^{3+}, \mathrm{Yb}^{3+}$ and $\mathrm{Tm}^{3+}, \mathrm{Yb}^{3+}$ Via Thermal Decomposition of Lanthanide Trifluoroacetate Precursors. J. Am. Chem. Soc. 2006, 128, 7444-7445.

(171) Zhang, M.; Fan, H.; Xi, B.; Wang, X.; Dong, C.; Qian, Y., Synthesis, Characterization, and Luminescence Properties of Uniform $\mathrm{Ln}^{3+}$-Doped $\mathrm{YF}_{3}$ nanospindles. J. Phys. Chem. C 2007, 111, 6652-6657.

(172) Benayas, A.; del Rosal, B.; Pérez-Delgado, A.; Santacruz-Gómez, K.; Jaque, D.; Hirata, G. A.; Vetrone, F., Nd:YAG Near-Infrared Luminescent Nanothermometers. Adv. Opt. Mater. 2015, 3, 687-694.

(173) Leiss, M., Nd ${ }^{3+}$ Photoluminescence in Semiconducting Binary Sesquisulphide Crystals of La, Gd and Y. J. Phys. C Solid State Phys. 1980, 13, 151-157.

(174) Lowe-Ma, C. K.; Vanderah, T. A.; Smith, T. E., The Ternary Yttrium Sulfides, $\mathrm{CaY}_{2} \mathrm{~S}_{4}$, $\mathrm{SrY}_{2} \mathrm{~S}_{4}$, and $\mathrm{BaY}_{2} \mathrm{~S}_{4}$ : Structures and Properties. J. Solid State Chem. 1995, 117, 363-372.

(175) Kroupa, D. M.; Roh, J. Y.; Milstein, T. J.; Creutz, S. E.; Gamelin, D. R., Quantum-Cutting YtterbiumDoped $\mathrm{CsPb}\left(\mathrm{Cl}_{1-x} \mathrm{Br}_{x}\right)_{3}$ Perovskite Thin Films with Photoluminescence Quantum Yields over 190\%. ACS Energy Lett. 2018, 3, 2390-2395.

(176) Zhao, Y.; Meek, G. A.; Levine, B. G.; Lunt, R. R., Near-Infrared Harvesting Transparent Luminescent Solar Concentrators. Adv. Opt. Mater. 2014, 2, 606-611.

(177) Ding, F.; Zhan, Y.; Lu, X.; Sun, Y., Recent Advances in Near-Infrared II Fluorophores for Multifunctional Biomedical Imaging. Chem. Sci. 2018, 9, 4370-4380.

(178) Skripka, A.; Morinvil, A.; Matulionyte, M.; Cheng, T.; Vetrone, F., Advancing Neodymium SingleBand Nanothermometry. Nanoscale 2019, 11, 11322-11330. 
(179) Hemmer, E.; Acosta-Mora, P.; Mendez-Ramos, J.; Fischer, S., Optical Nanoprobes for Biomedical Applications: Shining a Light on Upconverting and Near-Infrared Emitting Nanoparticles for Imaging, Thermal Sensing, and Photodynamic Therapy. J. Mater. Chem. B 2017, 5, 4365-4392.

(180) Brennan, J. G., Lanthanides: Sulfur, Selenium, and Tellurium Compounds. In Encyclopedia of Inorganic and Bioinorganic Chemistry, Wiley-VCH, Hoboken, New Jersey, 2012.

(181) Norris, D. J.; Yao, N.; Charnock, F. T.; Kennedy, T. A., High-Quality Manganese-Doped ZnSe Nanocrystals. Nano Lett. 2001, 1, 3-7.

(182) Archer, P. I.; Santangelo, S. A.; Gamelin, D. R., Direct Observation of sp-d Exchange Interactions in Colloidal $\mathrm{Mn}^{2+}$ - and $\mathrm{Co}^{2+}$-Doped CdSe Quantum Dots. Nano Lett. 2007, 7, 1037-1043.

(183) Panda, S. K.; Hickey, S. G.; Demir, H. V.; Eychmuller, A., Bright White-Light Emitting Manganese and Copper Co-Doped ZnSe Quantum Dots. Angew. Chem. Int. Ed. Engl. 2011, 50, 4432-4436.

(184) Bi, C.; Wang, S.; Li, Q.; Kershaw, S. V.; Tian, J.; Rogach, A. L., Thermally Stable Copper(II)-Doped Cesium Lead Halide Perovskite Quantum Dots with Strong Blue Emission. J. Phys. Chem. Lett. 2019, 10, 943-952.

(185) Kroupa, D. M.; Hughes, B. K.; Miller, E. M.; Moore, D. T.; Anderson, N. C.; Chernomordik, B. D.; Nozik, A. J.; Beard, M. C., Synthesis and Spectroscopy of Silver-Doped PbSe Quantum Dots. J. Am. Chem. Soc. 2017, 139, 10382-10394.

(186) Roy, S.; Tuinenga, C.; Fungura, F.; Dagtepe, P.; Chikan, V.; Jasinski, J., Progress toward Producing N-Type CdSe Quantum Dots: Tin and Indium Doped CdSe Quantum Dots. J. Phys. Chem. C 2009, 113, 13008-13015.

(187) Tuinenga, C.; Jasinski, J.; Iwamoto, T.; Chikan, V., In Situ Observation of Heterogeneous Growth of CdSe Quantum Dots: Effect of Indium Doping on the Growth Kinetics. ACS Nano 2008, 2, 1411 1421.

(188) Buonsanti, R.; Llordes, A.; Aloni, S.; Helms, B. A.; Milliron, D. J., Tunable Infrared Absorption and Visible Transparency of Colloidal Aluminum-Doped Zinc Oxide Nanocrystals. Nano Lett. 2011, 11, 4706-4710.

(189) Kim, J.-H.; Jang, E.-P.; Kwon, Y.; Jang, H. S.; Do, Y. R.; Yang, H., Enhanced Fluorescent Stability of Copper Indium Sulfide Quantum Dots through Incorporating Aluminum into ZnS Shell. J. Alloys Compd. 2016, 662, 173-178.

(190) Stavrinadis, A.; Konstantatos, G., Strategies for the Controlled Electronic Doping of Colloidal Quantum Dot Solids. ChemPhysChem 2016, 17, 632-644.

(191) Buonsanti, R.; Milliron, D. J., Chemistry of Doped Colloidal Nanocrystals. Chem. Mater. 2013, 25, 1305-1317.

(192) Pradhan, N.; Das Adhikari, S.; Nag, A.; Sarma, D. D., Luminescence, Plasmonic, and Magnetic Properties of Doped Semiconductor Nanocrystals. Angew. Chem. Int. Ed. Engl. 2017, 56, 7038-7054.

(193) Vlaskin, V. A.; Barrows, C. J.; Erickson, C. S.; Gamelin, D. R., Nanocrystal Diffusion Doping. J. Am. Chem. Soc. 2013, 135, 14380-14389.

(194) Tsujii, N.; Imanaka, Y.; Takamasu, T.; Kitazawa, H.; Kido, G., Photoluminescence Study of CulnS2. Phys. B Cond. Matter 2001, 298, 437-440.

(195) ten Kate, O. M.; Hintzen, H. T.; Dorenbos, P.; van der Kolk, E., Yb ${ }^{3+}$ Doped LaSi ${ }_{3} \mathrm{~N}_{5}$ and $\mathrm{YSi}_{3} \mathrm{~N}_{5}$ with Low Energy Charge Transfer for Near-Infrared Light-Emitting Diode and Solar Cell Application. J. Mater. Chem. 2011, 21, 18289-18294.

(196) Maeda, K.; Kawaida, N.; Tsudome, R.; Sakai, K.; Ikari, T., X-Ray and Photoluminescence Properties of $\mathrm{Sm}^{3+}$-Doped Barium Sulfide. Phys. Status Solidi C 2012, 9, 2271-2274.

(197) Erwin, S. C.; Zu, L.; Haftel, M. I.; Efros, A. L.; Kennedy, T. A.; Norris, D. J., Doping Semiconductor Nanocrystals. Nature 2005, 436, 91-94.

(198) Stavrinadis, A.; Pelli Cresi, J. S.; d'Acapito, F.; Magén, C.; Boscherini, F.; Konstantatos, G., Aliovalent Doping in Colloidal Quantum Dots and Its Manifestation on Their Optical Properties: Surface Attachment Versus Structural Incorporation. Chem. Mater. 2016, 28, 5384-5393.

(199) Justo, Y.; Sagar, L. K.; Flamee, S.; Zhao, Q.; Vantomme, A.; Hens, Z., Less Is More. Cation Exchange and the Chemistry of the Nanocrystal Surface. ACS Nano 2014, 8, 7948-7957. 
(200) Du, M. H.; Erwin, S. C.; Efros, A. L., Trapped-Dopant Model of Doping in Semiconductor Nanocrystals. Nano Lett. 2008, 8, 2878-2882.

(201) Chikan, V., Challenges and Prospects of Electronic Doping of Colloidal Quantum Dots: Case Study of CdSe. J. Phys. Chem. Lett. 2011, 2, 2783-2789.

(202) Wang, Z.; Meijerink, A., Concentration Quenching in Upconversion Nanocrystals. J. Phys. Chem. C Nanomater. Interfaces 2018, 122, 26298-26306.

(203) Johnson, N. J.; He, S.; Diao, S.; Chan, E. M.; Dai, H.; Almutairi, A., Direct Evidence for Coupled Surface and Concentration Quenching Dynamics in Lanthanide-Doped Nanocrystals. J. Am. Chem. Soc. 2017, 139, 3275-3282.

(204) Sun, Q.; Zheng, C.; Huston, L. Q.; Frankcombe, T. J.; Chen, H.; Zhou, C.; Fu, Z.; Withers, R. L.; Noren, L.; Bradby, J. E., et al., Bimetallic lons Codoped Nanocrystals: Doping Mechanism, Defect Formation, and Associated Structural Transition. J. Phys. Chem. Lett. 2017, 8, 3249-3255.

(205) Archer, P. I.; Santangelo, S. A.; Gamelin, D. R., Inorganic Cluster Syntheses of TM²+_Doped Quantum Dots (CdSe, CdS, CdSe/CdS): Physical Property Dependence on Dopant Locale. J. Am. Chem. Soc. 2007, 129, 9808-9818.

(206) Fainblat, R.; Barrows, C. J.; Gamelin, D. R., Single Magnetic Impurities in Colloidal Quantum Dots and Magic-Size Clusters. Chem. Mater. 2017, 29, 8023-8036.

(207) Lieberman, C. M.; Navulla, A.; Zhang, H.; Filatov, A. S.; Dikarev, E. V., Mixed-Ligand Approach to Design of Heterometallic Single-Source Precursors with Discrete Molecular Structure. Inorg. Chem. 2014, 53, 4733-4738.

(208) Zhang, Y. C.; Chen, W. W.; Hu, X. Y., Controllable Synthesis and Optical Properties of Zn-Doped CdS Nanorods from Single-Source Molecular Precursors. Cryst. Growth Des. 2007, 7, 580-586.

(209) Wang, C.; Gao, X.; Ma, Q.; Su, X., Aqueous Synthesis of Mercaptopropionic Acid Capped Mn_Doped ZnSe Quantum Dots. J. Mater. Chem. 2009, 19, 7016-7022.

(210) Wang, X.; Yan, X.; Li, W.; Sun, K., Doped Quantum Dots for White-Light-Emitting Diodes without Reabsorption of Multiphase Phosphors. Adv. Mater. 2012, 24, 2742-2747.

(211) Hildebrand, J. H.; Scott, R. L., The Solubility of Nonelectrolytes. $3^{\text {rd }}$ ed.; Reinhold Publishing Corporation: New York, 1950.

(212) Pradhan, N.; Goorskey, D.; Thessing, J.; Peng, X., An Alternative of CdSe Nanocrystal Emitters: Pure and Tunable Impurity Emissions in ZnSe Nanocrystals. J. Am. Chem. Soc. 2005, 127, 17586-17587.

(213) Chen, D.; Viswanatha, R.; Ong, G. L.; Xie, R.; Balasubramaninan, M.; Peng, X., Temperature Dependence of "Elementary Processes" in Doping Semiconductor Nanocrystals. J. Am. Chem. Soc. 2009, 131, 9333-9339.

(214) Capitani, C.; Pinchetti, V.; Gariano, G.; Santiago-Gonzalez, B.; Santambrogio, C.; Campione, M.; Prato, M.; Brescia, R.; Camellini, A.; Bellato, F., et al., Quantized Electronic Doping Towards Atomically Controlled "Charge-Engineered" Semiconductor Nanocrystals. Nano Lett. 2019, 19, 1307-1317.

(215) Makkar, M.; Saha, A.; Khalid, S.; Viswanatha, R., Thermodynamics of Dual Doping in Quantum Dots. J Phys Chem. Lett. 2019, 10, 1992-1998.

(216) Saha, A.; Chattopadhyay, S.; Shibata, T.; Viswanatha, R., Core-Shell to Doped Quantum Dots: Evolution of the Local Environment Using XAFS. J. Phys. Chem. C 2016, 120, 18945-18951.

(217) Rivest, J. B.; Jain, P. K., Cation Exchange on the Nanoscale: An Emerging Technique for New Material Synthesis, Device Fabrication, and Chemical Sensing. Chem. Soc. Rev. 2013, 42, 89-96.

(218) De Trizio, L.; Manna, L., Forging Colloidal Nanostructures Via Cation Exchange Reactions. Chem. Rev. 2016, 116, 10852-10887.

(219) Yang, Z.; Wei, M.; Voznyy, O.; Todorovic, P.; Liu, M.; Quintero-Bermudez, R.; Chen, P.; Fan, J. Z.; Proppe, A. H.; Quan, L. N., et al., Anchored Ligands Facilitate Efficient B-Site Doping in Metal Halide Perovskites. J. Am. Chem. Soc. 2019, 141, 8296-8305.

(220) Lesnyak, V.; Brescia, R.; Messina, G. C.; Manna, L., Cu Vacancies Boost Cation Exchange Reactions in Copper Selenide Nanocrystals. J. Am. Chem. Soc. 2015, 137, 9315-9323. 
(221) Mir, W. J.; Mahor, Y.; Lohar, A.; Jagadeeswararao, M.; Das, S.; Mahamuni, S.; Nag, A., Postsynthesis Doping of $\mathrm{Mn}$ and $\mathrm{Yb}$ into $\mathrm{CsPbX}_{3}(\mathrm{X}=\mathrm{Cl}, \mathrm{Br}$, or I) Perovskite Nanocrystals for Downconversion Emission. Chem. Mater. 2018, 30, 8170-8178.

(222) Sadtler, B.; Demchenko, D. O.; Zheng, H.; Hughes, S. M.; Merkle, M. G.; Dahmen, U.; Wang, L. W.; Alivisatos, A. P., Selective Facet Reactivity During Cation Exchange in Cadmium Sulfide Nanorods. J. Am. Chem. Soc. 2009, 131, 5285-5293.

(223) Hewavitharana, I. K.; Brock, S. L., When Ligand Exchange Leads to lon Exchange: Nanocrystal Facets Dictate the Outcome. ACS Nano 2017, 11, 11217-11224.

(224) Bullen, C.; Mulvaney, P., The Effects of Chemisorption on the Luminescence of CdSe Quantum Dots. Langmuir 2006, 22, 3007-3013.

(225) Zhang, K.; Mei, Q.; Guan, G.; Liu, B.; Wang, S.; Zhang, Z., Ligand Replacement-Induced Fluorescence Switch of Quantum Dots for Ultrasensitive Detection of Organophosphorothioate Pesticides. Anal. Chem. 2010, 82, 9579-9586.

(226) Kurzmann, A.; Ludwig, A.; Wieck, A. D.; Lorke, A.; Geller, M., Auger Recombination in SelfAssembled Quantum Dots: Quenching and Broadening of the Charged Exciton Transition. Nano Lett. 2016, 16, 3367-3372.

(227) Almeida, G.; Infante, l.; Manna, L., Resurfacing Halide Perovskite Nanocrystals. Science 2019, 364, 833-834.

(228) Smock, S. R.; Williams, T. J.; Brutchey, R. L., Quantifying the Thermodynamics of Ligand Binding to $\mathrm{CsPbBr}_{3}$ Quantum Dots. Angew. Chem. Int. Ed. Engl. 2018, 57, 11711-11715.

(229) Di Stasio, F.; Christodoulou, S.; Huo, N.; Konstantatos, G., Near-Unity Photoluminescence Quantum Yield in $\mathrm{CsPbBr}_{3}$ Nanocrystal Solid-State Films Via Postsynthesis Treatment with Lead Bromide. Chem. Mater. 2017, 29, 7663-7667.

(230) Bohn, B. J.; Tong, Y.; Gramlich, M.; Lai, M. L.; Doblinger, M.; Wang, K.; Hoye, R. L. Z.; MullerBuschbaum, P.; Stranks, S. D.; Urban, A. S., et al., Boosting Tunable Blue Luminescence of Halide Perovskite Nanoplatelets through Postsynthetic Surface Trap Repair. Nano Lett. 2018, 18, 5231 5238.

(231) Behera, R. K.; Das Adhikari, S.; Dutta, S. K.; Dutta, A.; Pradhan, N., Blue-Emitting CsPbCl Nanocrystals: Impact of Surface Passivation for Unprecedented Enhancement and Loss of Optical Emission. J. Phys. Chem. Lett. 2018, 9, 6884-6891.

(232) Xiao, Z.; Kerner, R. A.; Tran, N.; Zhao, L.; Scholes, G. D.; Rand, B. P., Engineering Perovskite Nanocrystal Surface Termination for Light-Emitting Diodes with External Quantum Efficiency Exceeding 15\%. Adv. Funct. Mater. 2019, 29, 1807284.

(233) Hassinen, A.; Moreels, I.; De Nolf, K.; Smet, P. F.; Martins, J. C.; Hens, Z., Short-Chain Alcohols Strip X-Type Ligands and Quench the Luminescence of PbSe and CdSe Quantum Dots, Acetonitrile Does Not. J. Am. Chem. Soc. 2012, 134, 20705-20712.

(234) Reiss, P.; Protiere, M.; Li, L., Core/Shell Semiconductor Nanocrystals. Small 2009, 5, 154-168.

(235) Jang, Y.; Shapiro, A.; Isarov, M.; Rubin-Brusilovski, A.; Safran, A.; Budniak, A. K.; Horani, F.; Dehnel, J.; Sashchiuk, A.; Lifshitz, E., Interface Control of Electronic and Optical Properties in IV-VI and II-VI Core/Shell Colloidal Quantum Dots: A Review. Chem. Commun. (Camb.) 2017, 53, 1002-1024.

(236) Chen, X.; Lou, Y.; Samia, A. C.; Burda, C., Coherency Strain Effects on the Optical Response of Core/Shell Heteronanostructures. Nano Lett. 2003, 3, 799-803.

(237) Rubin-Brusilovski, A.; Jang, Y.; Shapiro, A.; Safran, A.; Sashchiuk, A.; Lifshitz, E., Influence of Interfacial Strain on Optical Properties of PbSe/PbS Colloidal Quantum Dots. Chem. Mater. 2016, 28, 9056-9063.

(238) Berends, A. C.; van der Stam, W.; Hofmann, J. P.; Bladt, E.; Meeldijk, J. D.; Bals, S.; de Mello Donegá, C., Interplay between Surface Chemistry, Precursor Reactivity, and Temperature Determines Outcome of ZnS Shelling Reactions on CulnS 2 Nanocrystals. Chem. Mater. 2018, 30, 2400-2413.

(239) Wu, Y.; Wei, H.; Xu, L.; Cao, B.; Zeng, H., Progress and Perspective on CsPbX 3 Nanocrystals for Light Emitting Diodes and Solar Cells. J. Appl. Phys. 2020, 128, 050903. 
(240) Zhang, C.; Wang, S.; Li, X.; Yuan, M.; Turyanska, L.; Yang, X., Core/Shell Perovskite Nanocrystals: Synthesis of Highly Efficient and Environmentally Stable $\mathrm{FAPbBr}_{3} / \mathrm{CsPbBr}_{3}$ for LED Applications. Adv. Funct. Mater. 2020, 30, 1910582.

(241) Tang, X.; Yang, J.; Li, S.; Liu, Z.; Hu, Z.; Hao, J.; Du, J.; Leng, Y.; Qin, H.; Lin, X., et al., Single Halide Perovskite/Semiconductor Core/Shell Quantum Dots with Ultrastability and Nonblinking Properties. Adv. Sci. (Weinh) 2019, 6, 1900412.

(242) Ravi, V. K.; Saikia, S.; Yadav, S.; Nawale, V. V.; Nag, A., CsPbBr $3 / Z n S$ Core/Shell Type Nanocrystals for Enhancing Luminescence Lifetime and Water Stability. ACS Energy Lett. 2020, 5, 1794-1796.

(243) Zhong, Q.; Cao, M.; Hu, H.; Yang, D.; Chen, M.; Li, P.; Wu, L.; Zhang, Q., One-Pot Synthesis of Highly Stable CsPbBr ${ }_{3} @ \mathrm{SiO}_{2}$ Core-Shell Nanoparticles. ACS Nano 2018, 12, 8579-8587.

(244) Li, Z.-J.; Hofman, E.; Li, J.; Davis, A. H.; Tung, C.-H.; Wu, L.-Z.; Zheng, W., Photoelectrochemically Active and Environmentally Stable $\mathrm{CsPbBr} / 3 / \mathrm{TiO}_{2}$ core/Shell Nanocrystals. Adv. Funct. Mater. 2018, 28, 1704288.

(245) Modena, M. M.; Ruhle, B.; Burg, T. P.; Wuttke, S., Nanoparticle Characterization: What to Measure? Adv. Mater. 2019, 31, e1901556.

(246) Misra, N. L.; Singh Mudher, K. D., Total Reflection X-Ray Fluorescence: A Technique for Trace Element Analysis in Materials. Prog. Cryst. Growth Charact. Mater. 2002, 45, 65-74.

(247) Rabuffetti, F. A.; Culver, S. P.; Lee, J. S.; Brutchey, R. L., Local Structural Investigation of Eu ${ }^{3+}$-Doped $\mathrm{BaTiO}_{3}$ Nanocrystals. Nanoscale 2014, 6, 2909-2914.

(248) Sambasivam, S.; Kim, S. B.; Jeong, J. H.; Choi, B. C.; Lim, K. T.; Kim, S. S.; Song, T. K., Effect of Er ${ }^{3+}$ Doping in $\mathrm{SnO}_{2}$ Semiconductor Nanoparticles Synthesized by Solgel Technique. Curr. Appl. Phys. 2010, 10, 1383-1386.

(249) Larquet, C.; Nguyen, A. M.; Avila-Gutierrez, M.; Tinat, L.; Lassalle-Kaiser, B.; Gallet, J. J.; Bournel, F.; Gauzzi, A.; Sanchez, C.; Carenco, S., Synthesis of $\mathrm{Ce}_{2} \mathrm{O}_{2} \mathrm{~S}$ and $\mathrm{Gd}_{2(1-y)} \mathrm{Ce}_{2 y} \mathrm{O}_{2} \mathrm{~S}$ Nanoparticles and Reactivity from in Situ X-Ray Absorption Spectroscopy and X-Ray Photoelectron Spectroscopy. Inorg. Chem. 2017, 56, 14227-14236.

(250) Holmberg, V. C.; Helps, J. R.; Mkhoyan, K. A.; Norris, D. J., Imaging Impurities in Semiconductor Nanostructures. Chem. Mater. 2013, 25, 1332-1350.

(251) Terauchi, M., Transmission Electron Microscopy Characterization of Nanomaterials: Valence Electron Spectroscopy for Transmission Electron Microscopy. Springer-Verlag Berlin Heidelberg: Berlin, 2014.

(252) Nelson, H. D.; Gamelin, D. R., Valence-Band Electronic Structures of Cu+-Doped ZnS, Alloyed CuIn-Zn-S, and Ternary CulnS 2 Nanocrystals: A Unified Description of Photoluminescence across Compositions. J. of Phys. Chem. C 2018, 122, 18124-18133.

(253) Ferrer, M. M.; de Santana, Y. V.; Raubach, C. W.; La Porta, F. A.; Gouveia, A. F.; Longo, E.; Sambrano, J. R., Europium Doped Zinc Sulfide: A Correlation between Experimental and Theoretical Calculations. J. Mol. Model. 2014, 20, 2375.

(254) Brunet, G.; Marin, R.; Monk, M. J.; Resch-Genger, U.; Galico, D. A.; Sigoli, F. A.; Suturina, E. A.; Hemmer, E.; Murugesu, M., Exploring the Dual Functionality of an Ytterbium Complex for Luminescence Thermometry and Slow Magnetic Relaxation. Chem. Sci. 2019, 10, 6799-6808.

(255) Debnath, G. H.; Rudra, S.; Bhattacharyya, A.; Guchhait, N.; Mukherjee, P., Host Sensitized Lanthanide Photoluminescence from Post-Synthetically Modified Semiconductor Nanoparticles Depends on Reactant Identity. J. Colloid Interface Sci. 2019, 540, 448-465.

(256) Luo, W.; Liu, Y.; Chen, X., Lanthanide-Doped Semiconductor Nanocrystals: Electronic Structures and Optical Properties. Sci. China Mater. 2015, 58, 819-850.

(257) Binnemans, K., Interpretation of Europium(III) Spectra. Coord. Chem. Rev. 2015, 295, 1-45.

(258) Galico, D. A.; Marin, R.; Brunet, G.; Errulat, D.; Hemmer, E.; Sigoli, F. A.; Moilanen, J. O.; Murugesu, M., Triplet-State Position and Crystal-Field Tuning in Opto-Magnetic Lanthanide Complexes: Two Sides of the Same Coin. Chem. Eur. J. 2019, 25, 14625-14637.

(259) Ofoegbuna, T.; Bajgiran, K. R.; Kizilkaya, O.; Thomson, S. A. J.; Melvin, A. T.; Dorman, J. A., Photoluminescence Detection of Symmetry Transformations in Low-Dimensional Ferroelectric $\mathrm{ABO}_{3}$ Perovskites. J. Mater. Chem. C 2020, 8, 10767-10773. 
(260) Wurth, C.; Grabolle, M.; Pauli, J.; Spieles, M.; Resch-Genger, U., Relative and absolute determination of fluorescence quantum yields of transparent samples. Nat. Protoc. 2013, 8, 1535-1550.

(261) Chen, R., Glow Curves with General Order Kinetics. J. Electrochem. Soc. 1969, 116, 1254.

(262) Zhou, J.; Chizhik, A. I.; Chu, S.; Jin, D., Single-Particle Spectroscopy for Functional Nanomaterials. Nature 2020, 579, 41-50.

(263) Ostrowski, A. D.; Chan, E. M.; Gargas, D. J.; Katz, E. M.; Han, G.; Schuck, P. J.; Milliron, D. J.; Cohen, B. E., Controlled Synthesis and Single-Particle Imaging of Bright, Sub-10 nm Lanthanide-Doped Upconverting Nanocrystals. ACS Nano 2012, 6, 2686-2692.

(264) Yang, Y.; Chen, O.; Angerhofer, A.; Cao, Y. C., On Doping CdS/ZnS Core/Shell Nanocrystals with Mn. J. Am. Chem. Soc. 2008, 130, 15649-15661.

(265) Zhou, D.; Liu, D.; Pan, G.; Chen, X.; Li, D.; Xu, W.; Bai, X.; Song, H., Cerium and Ytterbium Codoped Halide Perovskite Quantum Dots: A Novel and Efficient Downconverter for Improving the Performance of Silicon Solar Cells. Adv. Mater. 2017, 29, 1704149.

(266) Yong, Z. J.; Guo, S. Q.; Ma, J. P.; Zhang, J. Y.; Li, Z. Y.; Chen, Y. M.; Zhang, B. B.; Zhou, Y.; Shu, J.; Gu, J. L., et al., Doping-Enhanced Short-Range Order of Perovskite Nanocrystals for near-Unity Violet Luminescence Quantum Yield. J. Am. Chem. Soc. 2018, 140, 9942-9951.

(267) Correa-Baena, J. P.; Saliba, M.; Buonassisi, T.; Gratzel, M.; Abate, A.; Tress, W.; Hagfeldt, A., Promises and Challenges of Perovskite Solar Cells. Science 2017, 358, 739-744.

(268) Kang, J.; Wang, L. W., High Defect Tolerance in Lead Halide Perovskite CsPbBr 3 . J. Phys. Chem. Lett. 2017, 8, 489-493.

(269) Zakutayev, A.; Caskey, C. M.; Fioretti, A. N.; Ginley, D. S.; Vidal, J.; Stevanovic, V.; Tea, E.; Lany, S., Defect Tolerant Semiconductors for Solar Energy Conversion. J. Phys. Chem. Lett. 2014, 5, $1117-$ 1125.

(270) Milstein, T. J.; Kluherz, K. T.; Kroupa, D. M.; Erickson, C. S.; De Yoreo, J. J.; Gamelin, D. R., Anion Exchange and the Quantum-Cutting Energy Threshold in Ytterbium-Doped $\mathrm{CsPb}_{2}\left(\mathrm{Cl}_{1-}{ }_{x} \mathrm{Br}_{x}\right)_{3}$ Perovskite Nanocrystals. Nano Lett. 2019, 19, 1931-1937.

(271) Ding, N.; Zhou, D.; Pan, G.; Xu, W.; Chen, X.; Li, D.; Zhang, X.; Zhu, J.; Ji, Y.; Song, H., EuropiumDoped Lead-Free $\mathrm{Cs}_{3} \mathrm{Bi}_{2} \mathrm{Br}_{9}$ Perovskite Quantum Dots and Ultrasensitive $\mathrm{Cu}^{2+}$ Detection. ACS Sust. Chem. Eng. 2019, 7, 8397-8404.

(272) Zhu, Y.; Zhu, J.; Song, H.; Huang, J.; Lu, Z.; Pan, G., Samarium Doping Improves Luminescence Efficiency of $\mathrm{Cs}_{3} \mathrm{Bi}_{2} \mathrm{Br}_{9}$ Perovskite Quantum Dots Enabling Efficient White Light-Emitting Diodes. J. Rare Earths 2020, DOI: 10.1016/j.jre.2020.06.007.

(273) Liu, Y.; Rong, X.; Li, M.; Molokeev, M. S.; Zhao, J.; Xia, Z., Incorporating Rare-Earth Terbium(III) Ions into $\mathrm{CS}_{2} \mathrm{Ag} \mathrm{InCl}_{6}: \mathrm{Bi}$ Nanocrystals toward Tunable Photoluminescence. Angew. Chem. Int. Ed. Engl. 2020, 132, 11731-11737.

(274) Chen, N.; Cai, T.; Li, W.; Hills-Kimball, K.; Yang, H.; Que, M.; Nagaoka, Y.; Liu, Z.; Yang, D.; Dong, A., et al., Yb- and Mn-Doped Lead-Free Double Perovskite $\mathrm{CS}_{2} \mathrm{AgBi}_{6}(\mathrm{X}=\mathrm{Cl}$;, Br) Nanocrystals. ACS Appl. Mater. Interfaces 2019, 11, 16855-16863.

(275) Lee, W.; Hong, S.; Kim, S., Colloidal Synthesis of Lead-Free Silver-Indium Double-Perovskite $\mathrm{Cs}_{2} \mathrm{AgInCl}_{6}$ Nanocrystals and Their Doping with Lanthanide lons. J. Phys. Chem. C 2019, 123, 26652672.

(276) Mahor, Y.; Mir, W. J.; Nag, A., Synthesis and near-Infrared Emission of Yb-Doped Cs 2 AgInCl 6 Double Perovskite Microcrystals and Nanocrystals. J. Phys. Chem. C 2019, 123, 15787-15793.

(277) Wang, C.-Y.; Liang, P.; Xie, R.-J.; Yao, Y.; Liu, P.; Yang, Y.; Hu, J.; Shao, L.; Sun, X. W.; Kang, F., et al., Highly Efficient Lead-Free (Bi,Ce)-Codoped $\mathrm{Cs}_{2} \mathrm{Ag}_{0.4} \mathrm{Na}_{0.6} \mathrm{InCl} \mathrm{I}_{6}$ Double Perovskites for White LightEmitting Diodes. Chem. Mater. 2020, 32, 7814-7821.

(278) Moon, B. J.; Kim, S. J.; Lee, S.; Lee, A.; Lee, H.; Lee, D. S.; Kim, T. W.; Lee, S. K.; Bae, S.; Lee, S. H., Rare-Earth-Element-Ytterbium-Substituted Lead-Free Inorganic Perovskite Nanocrystals for Optoelectronic Applications. Adv. Mater. 2019, 31, e1901716.

(279) Wang, J.; Zhu, Y.; He, N.; Sun, Y.; Grimes, C. A.; Cai, Q., Tri-Doped Alkaline Earth Sulfide Nanoparticles as a New Class of Highly Efficient Probe with Near-IR Stimulated Fluorescence for in Vivo and Ultrasensitive Bacteria Targeted Imaging. Sens. Actuators B Chem. 2020, 305, 127427. 
(280) Yu, Y.; Chen, G.; Zhou, Y.; Han, Z., Recent Advances in Rare-Earth Elements Modification of Inorganic Semiconductor-Based Photocatalysts for Efficient Solar Energy Conversion: A Review. J. Rare Earths 2015, 33, 453-462.

(281) Saqib, N. U.; Adnan, R.; Shah, I., A Mini-Review on Rare Earth Metal-Doped $\mathrm{TiO}_{2}$ for Photocatalytic Remediation of Wastewater. Environ. Sci. Pollut. Res. Int. 2016, 23, 15941-15951.

(282) Ong, C. B.; Ng, L. Y.; Mohammad, A. W., A Review of ZnO Nanoparticles as Solar Photocatalysts: Synthesis, Mechanisms and Applications. Renew. Sust. Energ. Rev. 2018, 81, 536-551.

(283) Kumar, V.; Ntwaeaborwa, O. M.; Soga, T.; Dutta, V.; Swart, H. C., Rare Earth Doped Zinc Oxide Nanophosphor Powder: A Future Material for Solid State Lighting and Solar Cells. ACS Photonics 2017, 4, 2613-2637.

(284) Wu, N.; Luo, Q.; Qiao, X.; Ma, C.-Q., The Preparation of a Eu+3+-Doped ZnO Bi-Functional Layer and Its Application in Organic Photovoltaics. Mater. Res. Express 2015, 2, 125901.

(285) Yao, N.; Huang, J.; Fu, K.; Deng, X.; Ding, M.; Shao, M.; Xu, X., Enhanced Light Harvesting of DyeSensitized Solar Cells with Up/Down Conversion Materials. Electrochim. Acta 2015, 154, 273-277.

(286) Wolska, E.; Kaszewski, J.; Kiełbik, P.; Grzyb, J.; Godlewski, M. M.; Godlewski, M., Rare Earth Activated ZnO Nanoparticles as Biomarkers. Opti. Mater. 2014, 36, 1655-1659.

(287) Carofiglio, M.; Barui, S.; Cauda, V.; Laurenti, M., Doped Zinc Oxide Nanoparticles: Synthesis, Characterization and Potential Use in Nanomedicine. Appl. Sci. 2020, 10, 5194.

(288) Sun, L.-W.; Shi, H.-Q.; Li, W.-N.; Xiao, H.-M.; Fu, S.-Y.; Cao, X.-Z.; Li, Z.-X., Lanthanum-Doped ZnO Quantum Dots with Greatly Enhanced Fluorescent Quantum Yield. J. Mater. Chem. 2012, 22, 82218227.

(289) Green, M. A.; Ho-Baillie, A.; Snaith, H. J., The Emergence of Perovskite Solar Cells. Nat. Photonics 2014, 8, 506-514.

(290) Wang, L.; Zhou, H.; Hu, J.; Huang, B.; Sun, M.; Dong, B.; Zheng, G.; Huang, Y.; Chen, Y.; Li, L., et al., $\mathrm{A} \mathrm{Eu}^{3+}-\mathrm{Eu}^{2+}$ Ion Redox Shuttle Imparts Operational Durability to Pb-I Perovskite Solar Cells. Science 2019, 363, 265-270.

(291) Duan, J.; Zhao, Y.; Yang, X.; Wang, Y.; He, B.; Tang, Q., Lanthanide lons Doped CsPbBr 3 Halides for $\mathrm{Htm}$-Free 10.14\%-Efficiency Inorganic Perovskite Solar Cell with an Ultrahigh Open-Circuit Voltage of 1.594 V. Adv. Energy Mater. 2018, 8, 1802346.

(292) Nelson, C. A.; Monahan, N. R.; Zhu, X. Y., Exceeding the Shockley-Queisser Limit in Solar Energy Conversion. Energy Environ. Sci. 2013, 6, 3508-3519.

(293) Ding, N.; Xu, W.; Zhou, D.; Pan, G.; Li, D.; Ji, Y.; Chen, X.; Yang, D.; Bai, X.; Ma, C.-G., et al., Upconversion Ladder Enabled Super-Sensitive Narrowband Near-Infrared Photodetectors Based on Rare Earth Doped Florine Perovskite Nanocrystals. Nano Energy 2020, 76, 105103.

(294) Cai, T.; Wang, J.; Li, W.; Hills-Kimball, K.; Yang, H.; Nagaoka, Y.; Yuan, Y.; Zia, R.; Chen, O., $\mathrm{Mn}^{2+} / \mathrm{Yb}^{3+}$ Codoped $\mathrm{CsPbCl}_{3}$ Perovskite Nanocrystals with Triple-Wavelength Emission for Luminescent Solar Concentrators. Adv. Sci. 2020, 23, 2001317.

(295) Luo, X.; Ding, T.; Liu, X.; Liu, Y.; Wu, K., Quantum-Cutting Luminescent Solar Concentrators Using Ytterbium-Doped Perovskite Nanocrystals. Nano Lett. 2019, 19, 338-341.

(296) Cohen, T. A.; Milstein, T. J.; Kroupa, D. M.; MacKenzie, J. D.; Luscombe, C. K.; Gamelin, D. R., Quantum-cutting $\mathrm{Yb}^{3+}$-doped perovskite nanocrystals for monolithic bilayer luminescent solar concentrators. J. Mater. Chem. A 2019, 7, 9279-9288.

(297) Zhou, Y.; Benetti, D.; Fan, Z.; Zhao, H.; Ma, D.; Govorov, A. O.; Vomiero, A.; Rosei, F., Near Infrared, Highly Efficient Luminescent Solar Concentrators. Adv. Energy Mater. 2016, 6, 1501913.

(298) Erickson, C. S.; Crane, M. J.; Milstein, T. J.; Gamelin, D. R., Photoluminescence Saturation in Quantum-Cutting $\mathrm{Yb}^{3+}$-Doped $\mathrm{CsPb}\left(\mathrm{Cl}_{1-x} \mathrm{Br}_{x}\right)_{3}$ Perovskite Nanocrystals: Implications for Solar Downconversion. J. Phys. Chem. C 2019, 123, 12474-12484.

(299) Pan, G.; Bai, X.; Xu, W.; Chen, X.; Zhou, D.; Zhu, J.; Shao, H.; Zhai, Y.; Dong, B.; Xu, L., et al., Impurity Ions Codoped Cesium Lead Halide Perovskite Nanocrystals with Bright White Light Emission toward Ultraviolet-White Light-Emitting Diode. ACS Appl. Mater. Interfaces 2018, 10, 39040-39048. 
(300) Yao, J. S.; Ge, J.; Han, B. N.; Wang, K. H.; Yao, H. B.; Yu, H. L.; Li, J. H.; Zhu, B. S.; Song, J. Z.; Chen, C., et al., Ce ${ }^{3+}$-Doping to Modulate Photoluminescence Kinetics for Efficient $\mathrm{CsPbBr}_{3}$ Nanocrystals Based Light-Emitting Diodes. J. Am. Chem. Soc. 2018, 140, 3626-3634.

(301) Lin, P.; Chen, H.; Wei, Z.; Lin, Y.; Lin, J.; Chen, Y.; Cheng, Z., Continuous-Flow Synthesis of Doped All-Inorganic Perovskite Nanocrystals Enabled by a Microfluidic Reactor for Light-Emitting Diode Application. Sci. China Mater. 2020, 63, 1526-1536.

(302) Ren, F.; Liu, H.; Zhang, H.; Jiang, Z.; Xia, B.; Genevois, C.; He, T.; Allix, M.; Sun, Q.; Li, Z., et al., Engineering NIR-Ilb Fluorescence of Er-Based Lanthanide Nanoparticles for through-Skull Targeted Imaging and Imaging-Guided Surgery of Orthotopic Glioma. Nano Today 2020, 34, 100905.

(303) Li, Y.; Zeng, S.; Hao, J., Non-Invasive Optical Guided Tumor Metastasis/Nessel Imaging by Using Lanthanide Nanoprobe with Enhanced Down-Shifting Emission Beyond 1500 nm. ACS Nano 2019, 13, 248-259.

(304) Dong, N. N.; Pedroni, M.; Piccinelli, F.; Conti, G.; Sbarbati, A.; Ramirez-Hernandez, J. E.; Maestro, L. M.; Iglesias-de la Cruz, M. C.; Sanz-Rodriguez, F.; Juarranz, A., et al., NIR-to-NIR Two-Photon Excited $\mathrm{CaF}_{2}: \mathrm{Tm}^{3+}, \mathrm{Yb}^{3+}$ Nanoparticles: Multifunctional Nanoprobes for Highly Penetrating Fluorescence Bio-Imaging. ACS Nano 2011, 5, 8665-8671.

(305) Hou, Z.; Deng, K.; Li, C.; Deng, X.; Lian, H.; Cheng, Z.; Jin, D.; Lin, J., 808 Nm Light-Triggered and Hyaluronic Acid-Targeted Dual-Photosensitizers Nanoplatform by Fully Utilizing $\mathrm{Nd}^{3+}$-Sensitized Upconversion Emission with Enhanced Anti-Tumor Efficacy. Biomaterials 2016, 101, $32-46$.

(306) Chen, S.; Weitemier, A. Z.; Zeng, X.; He, L.; Wang, X.; Tao, Y.; Huang, A. J. Y.; Hashimotodani, Y.; Kano, M.; Iwasaki, H., et al., Near-Infrared Deep Brain Stimulation Via Upconversion NanoparticleMediated Optogenetics. Science 2018, 359, 679-684.

(307) All, A. H.; Zeng, X.; Teh, D. B. L.; Yi, Z.; Prasad, A.; Ishizuka, T.; Thakor, N.; Hiromu, Y.; Liu, X., Expanding the Toolbox of Upconversion Nanoparticles for in Vivo Optogenetics and Neuromodulation. Adv. Mater. 2019, 31, e1803474.

(308) Hemmer, E.; Benayas, A.; Legare, F.; Vetrone, F., Exploiting the Biological Windows: Current Perspectives on Fluorescent Bioprobes Emitting above 1000 nm. Nanoscale Horiz. 2016, 1, 168184.

(309) Li, Z.; Ding, X.; Cong, H.; Wang, S.; Yu, B.; Shen, Y., Recent Advances on Inorganic LanthanideDoped NIR-II Fluorescence Nanoprobes for Bioapplication. J. Lumin. 2020, 228, 117627.

(310) Fan, Y.; Zhang, F., A New Generation of NIR-II Probes: Lanthanide-Based Nanocrystals for Bioimaging and Biosensing. Adv. Opt. Mater. 2019, 7, 1801417.

(311) Skripka, A.; Marin, R.; Benayas, A.; Canton, P.; Hemmer, E.; Vetrone, F., Covering the Optical Spectrum through Collective Rare-Earth Doping of $\mathrm{NaGdF}_{4}$ Nanoparticles: 806 and $980 \mathrm{~nm}$ Excitation Routes. Phys. Chem. Chem. Phys. 2017, 19, 11825-11834.

(312) Liu, Y.; Luo, W.; Li, R.; Zhu, H.; Chen, X., Near-Infrared Luminescence of $\mathrm{Nd}^{3+}$ and $\mathrm{Tm}^{3+}$ Ions Doped ZnO Nanocrystals. Opt. Express 2009, 17, 9748-9753.

(313) Hinsch, A.; Lohmann, S.-H.; Strelow, C.; Kipp, T.; Würth, C.; Geißler, D.; Kornowski, A.; Wolter, C.; Weller, H.; Resch-Genger, U., et al., Fluorescence Quantum Yield and Single-Particle Emission of CdSe Dot/CdS Rod Nanocrystals. J. Phys. Chem. C 2019, 123, 24338-24346.

(314) Stroyuk, O.; Weigert, F.; Raevskaya, A.; Spranger, F.; Würth, C.; Resch-Genger, U.; Gaponik, N.; Zahn, D. R. T., Inherently Broadband Photoluminescence in Ag-In-S/ZnS Quantum Dots Observed in Ensemble and Single-Particle Studies. J. Phys. Chem. C 2019, 123, 2632-2641.

(315) Cichy, B.; Rich, R.; Olejniczak, A.; Gryczynski, Z.; Strek, W., Two Blinking Mechanisms in Highly Confined $\mathrm{AgInS}_{2}$ and $\mathrm{AgInS}_{2} / \mathrm{ZnS}$ Quantum Dots Evaluated by Single Particle Spectroscopy. Nanoscale 2016, 8, 4151-4159.

(316) Ryan, K.; Lengyel, J.; Shatruk, M., Crystal Structure Prediction Via Deep Learning. J. Am. Chem. Soc. 2018, 140, 10158-10168.

(317) Toso, S.; Akkerman, Q. A.; Martin-Garcia, B.; Prato, M.; Zito, J.; Infante, I.; Dang, Z.; Moliterni, A.; Giannini, C.; Bladt, E., et al., Nanocrystals of Lead Chalcohalides: A Series of Kinetically Trapped Metastable Nanostructures. J. Am. Chem. Soc. 2020, 142, 10198-10211. 


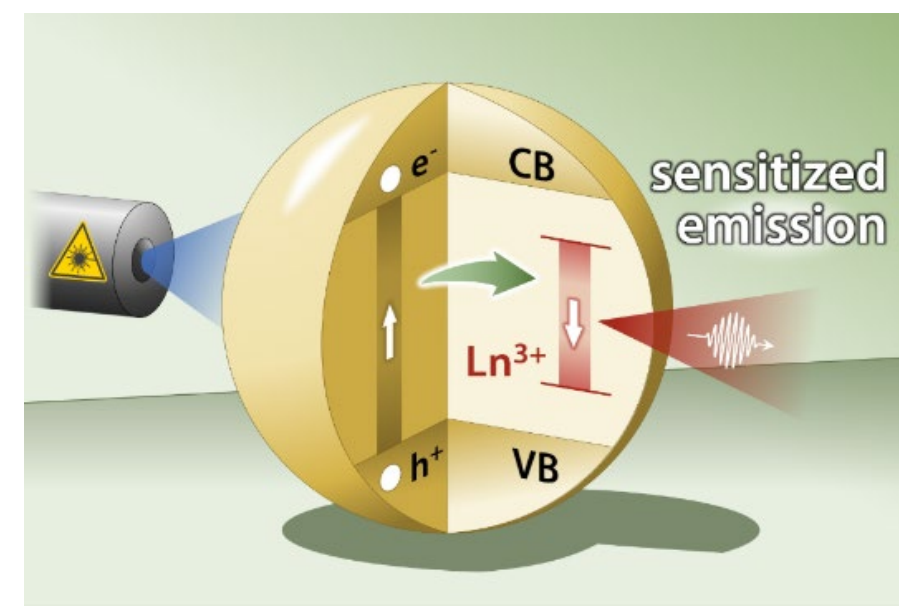

Authors'BIO:

Riccardo Marin obtained his PhD in Chemistry jointly from the University Ca' Foscari (Venice, Italy) and the Institut National de la Recherche Scientifique (INRS — Varénnes, Canada) under the supervision of Prof. P. Canton and Prof. F. Vetrone. He then undertook a postdoctoral fellowship at the University of Ottawa from 2017 to 2019 with Prof. E. Hemmer and Prof. M. Murugesu. He is currently a Marie Skłodowska-Curie fellow at the Universidad Autónoma de Madrid in the group of Prof. D. Jaque. His research interests encompass the development and study of optically active (nano)materials based on lanthanide ions and semiconductors.

Daniel Jaque (PhD in Physics, '99) is currently Associate (tenured) Professor in the Department of Material Physics at Universidad Autónoma de Madrid, co-director of the Fluorescence Imaging Group (FIG), and Director of the Nanobiology Group at "Ramón y Cajal" Health Research Institute. His current research focus lies in multimodal in vivo imaging and use of hyperspectral microscopy techniques for biological sensing. Prof. Jaque is a recognized pioneer and worldwide renowned authority in the field of luminescent nanothermometry, especially regarding biomedical implementation of this fastgrowing research field. 